FRP CONFINED REINFORCED CONCRETE CIRCULAR CROSS SECTION SEISMIC APPLICATIONS

\author{
A Thesis \\ Presented to \\ the Faculty of California Polytechnic State University, \\ San Luis Obispo \\ In Partial Fulfillment \\ of the Requirements for the Degree \\ Master of Science in Civil and Environmental Engineering
}

by

Jeffrey Gordon Lyon

August 2009 
(C) 2009

Jeffrey Gordon Lyon

ALL RIGHTS RESERVED 
COMMITTEE MEMBERSHIP

TITLE: FRP CONFINED REINFORCED CONCRETE CIRCULAR CROSS SECTION SEISMIC APPLICATIONS

AUTHOR: Jeffrey Gordon Lyon

DATE SUBMITTED: August 2009

COMMITTEE CHAIR: Charles B. Chadwell, Assistant Professor

COMMITTEE MEMBER: Rakesh K. Goel, Professor \& Chair

COMMITTEE MEMBER: Garrett J. Hall, Associate Professor 


\title{
ABSTRACT \\ FRP CONFINED REINFORCED CONCRETE CIRCULAR CROSS SECTION SEISMIC APPLICATIONS
}

by

\author{
Jeffrey Gordon Lyon
}

In recent earthquakes, structures have not performed as well as expected resulting in a need for better means of retrofitting and improvements in seismic design. Fiber Reinforced Polymers (FRP), as a material with potential to increase strength and ductility of columns in conjunction with capacity design methodology, has promise for seismic design. By investigating the displacement, ductility, and flexural strength properties of FRP confined reinforced concrete circular cross sections, this study analyzes the seismic applications of FRP confinement.

The study is performed by incorporating an FRP confined concrete stress-strain model into a developed Moment-Curvature and PM Interaction software. This software conducts a comparison between traditional steel and FRP confined sections while performing parameter studies on the 28-day unconfined concrete compressive strength, longitudinal reinforcing ratio, cross section diameter, FRP confinement jacket thickness-cross section diameter ratio, and FRP confinement system design variables. These studies validate FRP's performance for seismic applications resulting in several design recommendations to increase displacement capacity, ductility, and flexural strength and, thus, seismic performance. 


\section{ACKNOWLEDGMENTS}

I would like to thank all my family, friends, and instructors. Without their support and guidance this would not have been possible. 


\section{TABLE OF CONTENTS}

LIST OF TABLES viii

LIST OF FIGURES Ix ix

1 Introduction 1

2 Literature Review 5

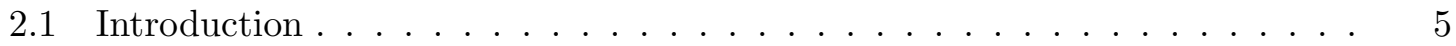

2.2 Stress-Strain Relationships . . . . . . . . . . . . . . . 8

2.3 FRP Confinement and Stress-Strain Properties . . . . . . . . . . . . . 11

2.4 FRP Confinement Models . . . . . . . . . . . . . . . . . . . . . . 18

2.4.1 Saadatmanesh et al. (1994) Steel Based Confinement Model . . . . . 18

2.4.2 Samaan et al. (1998) Empirical Confinement Model . . . . . . . . . 19

2.4.3 Saiidi et al. (2005) Bilinear Confinement Model . . . . . . . . . . . . 21

2.5 Selected Confinement Model . . . . . . . . . . . . . . . . . . . . . 22

3 Moment-Curvature and Axial Force-Moment Interaction 25

3.1 Introduction . . . . . . . . . . . . . . . . . . 25

3.2 Moment-Curvature Analysis Methodology . . . . . . . . . . . . . . . . 27

3.3 Axial Force-Moment Interaction Analysis Methodology . . . . . . . . . . . . 34

3.4 Conclusion . . . . . . . . . . . . . . . . . . . . 36

4 Confinement Comparison and Parameter Study 38

4.1 Introduction . . . . . . . . . . . . . . . . . . . . . . 38

4.2 Confinement Comparison . . . . . . . . . . . . . . . . 44

4.3 Parameter Study . . . . . . . . . . . . . . . . . . . . . . . 51

4.3.1 Unconfined Concrete Compressive Strength Parameter Study . . . . 53

4.3.2 Longitudinal Reinforcing Ratio Parameter Study . . . . . . . . . . . 56

4.3.3 Varying Cross Section Diameter Parameter Study . . . . . . . . . . 59

4.3.4 FRP confinement Jacket Thickness-Cross Section Diameter Ratio Parameter Study . . . . . . . . . . . . . . . . 62

4.3.5 Varying FRP System Parameter Study . . . . . . . . . . . . . . 63 
$\begin{array}{ll}\text { BIBLIOGRAPHY } & 67\end{array}$

A Parameter Study Figures 72

B $\quad$ MC and PM Interaction Software 78

B.1 Driver Script for MC and PM Interaction Analyses . . . . . . . . . . . . 78

B.2 Axial Load Input Script . . . . . . . . . . . . . . . . . . . . . . 80

B.3 Unconfined Concrete Material Properties Input Script . . . . . . . . . . . 81

B.4 Cross Section Diameter Input Script . . . . . . . . . . . . . . . . . . 82

B.5 FRP System Parameters Input Script _. . . . . . . . . . . . . . . 83

B.6 Material Model Selection Script . . . . . . . . . . . . . . . . . . . 84

B.7 Longitudinal Rebar Input Script . . . . . . . . . . . . . . . . . 86

B.8 Reinforcing Steel Material Properties Input Script . . . . . . . . . . . . . 87

B.9 Transverse Reinforcement Detail Input Script . . . . . . . . . . . . . . . . 89

B.10 Layer Centroid Strain for Moment-Curvature Analyses . . . . . . . . . . . . 90

B.11 Layer Centrior Strain for PM Interaction Analyses . . . . . . . . . . . . . . 92

B.12 Confined Concrete Stress . . . . . . . . . . . . . . . . . . . . 94

B.13 Unconfined Concrete Stress . . . . . . . . . . . . . . . . . . . . 95

B.14 Ultimate Failure Strains _. . . . . . . . . . . . . . . . . . . . . 95

B.15 Longitudinal Reinforcing Steel Area . . . . . . . . . . . . . . . . 96

B.16 Longitudinal Reinforcing Steel Ratio . . . . . . . . . . . . . . . . . 97

B.17 Maximum Moment . . . . . . . . . . . . . . . . . . . . . 97 97

B.18 Reaction Moment . . . . . . . . . . . . . . . . . . . . . 98

B.19 Moment-Curvature Analysis . . . . . . . . . . . . . . . . . . . . . . 99

B.20 MC Calculations for PM Interaction Analysis . . . . . . . . . . . . . . 100

B.21 Reaction Force . . . . . . . . . . . . . . . . . . . . . . 102

B.22 Gross Section Area . . . . . . . . . . . . . . . . . . . . . 103

B.23 Cross Section Properties . . . . . . . . . . . . . . . . . . . . . 103

B.24 Longitudinal Reinforcement Stress . . . . . . . . . . . . . . . . . . 115

B.25 Ultimate Curvature . . . . . . . . . . . . . . . . . . . . . . 116

B.26 PM Interaction Diagram . . . . . . . . . . . . . . . . . . 117

B.27 Load Check . . . . . . . . . . . . . . . . . . . . . . . . . . . . . 118

B.28 Mander Confined Concrete Material Model . . . . . . . . . . . . . . . . . . 119

B.29 Mander Unconfined Concrete Material Model . . . . . . . . . . . . . . . . . 120

B.30 Moment-Curvature Plot . . . . . . . . . . . . . . . . . . . . . . . . . . . 122

B.31 Material Model Stress-Strain Plots . . . . . . . . . . . . . . . . . . . . . 123

B.32 Priestley Bi-Axial Steel Model . . . . . . . . . . . . . . . . . . . . . . . 124

B.33 Rebar Properties . . . . . . . . . . . . . . . . . . . . . 125

B.34 Samaan Confined Concrete Material Model . . . . . . . . . . . . . . . 126

B.35 Section Plot . . . . . . . . . . . . . . . . . . . . 128

B.36 FRP and Steel Material Stress-Strain Plots . . . . . . . . . . . . . . . . 132

B.37 Cross Section Failure Strain Plot . . . . . . . . . . . . . . . . . . 134

B.38 Cross Section Failure Stress Plot . . . . . . . . . . . . . . 137 


\section{LIST OF TABLES}

3.1 Discretization Sensitivity Study Cross Section Configuration . . . . . . . . 29

4.1 Confinement Comparison Cross Section Configurations . . . . . . . . . . . 45

4.2 Parameter Study Cross Section Configurations . . . . . . . . . . . . . 54

4.3 FRP Confinement System Properties . . . . . . . . . . . . . . . . 55

4.4 Effects of Increasing the $f^{\prime} c$ Parameter . . . . . . . . . . . . . 55

4.5 Effects of Increasing the $\frac{A_{s t}}{A_{g}}$ Parameter . . . . . . . . . . . . . . . . 57

4.6 Effects of Increasing the $D^{A_{g}}$ Parameter $\ldots \ldots \ldots \ldots$

4.7 Effects of Increasing the $\frac{n t}{D}$ Parameter . . . . . . . . . . . . . . 61

4.8 FRP System Property $\left(f_{f u}\right.$ or $\left.e_{f u}\right)$ with Greatest Influence on Cross Section Properties .......................... 63 


\section{LIST OF FIGURES}

2.1 Steel and FRP Stress-Strain Relationships . . . . . . . . . . . . . . . . . 14

2.2 Concrete Stress-Strain Relationships . . . . . . . . . . . . . . . . . 15

2.3 FRP Confinement Free-Body Diagram . . . . . . . . . . . . . . 16

3.1 Discretization Sensitivity Analysis . . . . . . . . . . . . . . . . . 29

3.2 Discretization Sensitivity Error Analysis . . . . . . . . . . . . . . . 30

3.3 Cross Section Materials . . . . . . . . . . . . . . . . . . . . 31

3.4 Cross Section Strain Distribution . . . . . . . . . . . . . . . . . 32

3.5 Cross Section Stress Distribution . . . . . . . . . . . . . . . . 33

3.6 Influence of Axial Load on Moment-Curvature Relationship . . . . . . . . . 35

3.7 Ultimate Moment PM Interaction Curve . . . . . . . . . . . . . . . . . . 36

4.1 Steel and FRP Confinement Comparison Figures . . . . . . . . . . . . . . . 46

A.1 $f_{c}^{\prime}$ Parameter Study for FRP Confined Concrete . . . . . . . . . . . . . . . 73

A.2 $\frac{A_{s t}}{A_{g}}$ Parameter Study for FRP Confined Concrete . . . . . . . . . . . . . . 74

A.3 $\stackrel{A_{g}}{D}$ Parameter Study for FRP Confined Concrete . . . . . . . . . . . . . . . 75

A.4 $\frac{n t}{D}$ Parameter Study for FRP Confined Concrete . . . . . . . . . . . 76

A.5 System Parameter Study for FRP Confined Concrete . . . . . . . . . . 77 


\section{CHAPTER 1}

\section{Introduction}

Fiber reinforced polymer (FRP) composites have been used as longitudinal reinforcement, admixtures, and fiber wraps to improve flexure, shear and compressive strengths of reinforced concrete. In past years, due to high cost and lack of product knowledge, the use of FRP composites had been limited at best. Recent cost decreases, however, have allowed FRP composites to become a feasible option for structural applications resulting in an increase in research on this topic. Previous FRP composite findings have shown that their high strength can help make reinforced concrete cross sections stronger and more ductile. It has also been found that due to their minimal thickness, light weight, and corrosion resistive properties, FRP composites can increase the efficiency of a structure.

FRP confinement's ability to increase the strength, ductility, and efficiency of reinforced concrete gives it good potential in seismic design. For this reason, this study investigates FRP confined reinforced concrete circular cross sections to provide conclusions that will aid in both the seismic design of existing structure rehabilitation as well as the 
seismic design of new structures.

In seismic design, locations of plastic hinges must be identified. Next, from member displacement, ductility, and flexural demands, the required inelastic rotation, ductility, flexural strength, and flexural overstrength of the plastic hinges are determined. With the required strength of the plastic hinges known, the forces in the other non-ductile sections are calculated using an adequate strength margin between non-ductile sections and the maximum feasible overstrength of the plastic hinges to ensure the non-ductile failure mode is avoided. Once the strengths of all the non-ductile sections are established they can then be detailed [24].

Due to the relationship between member and cross section properties, this study focuses on cross section rotational capacity, ductility, and flexural strength as well as potential overstrength of FRP confined plastic hinges to provide insight of how FRP confined members and structures will react to seismic loading. To do this, Moment-Curvature analyses are used to evaluate the rotational capacity, ductility, and flexural strength of a cross section. Axial Load-Moment Interaction analyses are used to evaluate the possible overstrength of plastic hinges considering feasible variations in axial loads.

Throughout this study, FRP confined reinforced concrete cross sections are compared to traditional steel confined cross sections while analyzing the effect typical design variables have on the curvature capacity, ductility, flexural strength, and flexural overstrength of the FRP confined cross sections. Though transverse reinforcement also enhances shear strength, this study only focuses on the confinement effects of FRP composites on reinforced concrete. Specially developed software is used to conduct a series of Moment- 
Curvature and Axial Force-Moment Interaction analyses on various reinforced concrete circular cross section configurations subjected to axial loading and uni-axial bending. The results from these analyses are then used to compare the steel confined and FRP confined cross sections and to determine the effects the design variables have on the FRP confined sections.

The study presented herein is broken into five chapters with an introduction in the first Chapter. Chapter 2 discusses active and passive confinement of reinforced concrete. FRP composites are presented as a confinement mechanism with associated stress-strain characteristics. This chapter also investigates previous research on FRP confined concrete stress-strain material models for their use in the software developed for this study. Chapter 3 introduces Moment-Curvature and Axial Force-Moment Interaction analyses. The methodology for both is explained and the software that will be used to conduct Moment-Curvature and Axial Force-Moment Interaction analyses for a series of parameter studies is developed and verified. Then, in Chapter 4, the software to conduct comparison between steel confined and FRP confined concrete is utilized, and a series of design variable parameter studies are performed. The comparison is conducted to establish whether FRP materials can confine reinforced concrete as effectively as steel and to identify key differences between the two confining systems that are critical for seismic resistance. The parameter study variables include the 28-day unconfined concrete strength, longitudinal reinforcing ratio, cross section diameter, FRP confinement jacket thickness-cross section diameter ratio, and FRP confinement system. The studies are used to demonstrate the effect each design variable has on the cross section's curvature capacity, ductility, flexural strength, and flexural overstrength. In 
addition, the parameter studies are performed so that FRP confined plastic hinges can be precisely detailed to achieve the desired rotation, ductility, and flexural strength necessary to resist series of seismic inelastic deformations. From there, a strength hierarchy can be established so that non-ductile members are designed with enough strength to remain in the elastic range during seismic activity. Conclusions drawn from this study are presented in Chapter 5. 


\section{CHAPTER 2}

\section{Literature Review}

\section{$2.1 \quad$ Introduction}

Ductility in the plastic hinge regions of both new and retrofitted reinforced concrete columns requires special consideration in seismic design. Under large seismic excitation, proper ductility in the plastic hinge region will ensure that the column can deform inelastically through a series of displacement response cycles without significant loss of strength. The most important design consideration for ductility in the plastic hinge region of reinforced concrete columns is the transverse reinforcement detail. Properly designed transverse reinforcement ensures that the concrete in compression is adequately confined, the longitudinal reinforcement does not buckle, and a brittle shear failure is avoided [17]. This study focuses on the confinement aspect of transverse reinforcement in reinforced concrete columns.

The confinement of reinforced concrete can either be active or passive. Active confinement is a transverse stress applied around a cross section that is always present 
regardless of the section's loading conditions. This can be achieved by encasing a reinforced concrete member with a jacket that is slightly larger than the cross section. The void between the jacket and the concrete surface is then filled with pressure grout. Active confinement can also be achieved by prestressing the confinement jacket as it is applied around the concrete member.

In 1906, Considère [10] found that the application of active confinement on concrete test specimens increased their strength and ductility. Early research with concrete confined by hydrostatic fluid pressure (active confinement) showed that the maximum concrete stress $\left(f_{c c}^{\prime}\right)$ and corresponding longitudinal strain $\left(\varepsilon_{c c}\right)$ could be represented by Equations 2.1 and 2.2, respectively, with $f_{l}, f_{c o}^{\prime}$, and $\varepsilon_{c o}$ representing lateral fluid pressure, unconfined concrete strength, and corresponding strain, respectively. Coefficients $k_{1}$ and $k_{2}$ are parameters that describe confinement efficiency and are functions of both concrete strength and lateral pressure [17]. In 1928 Richart et al. [25] tested concrete cylinders under three different active confinement pressures and found the average value of $k_{1}$ to be 4.1 and $k_{2}$ to be $5 k_{1}(20.5)$.

$$
\begin{gathered}
f_{c c}^{\prime}=f_{c o}^{\prime}+k_{1} f_{l} \\
\varepsilon_{c c}=\varepsilon_{c o}\left(1+k_{2} \frac{f_{l}}{f_{c o}^{\prime}}\right)
\end{gathered}
$$

The second confinement type, passive confinement, is achieved by adding transverse reinforcement to the concrete cross section that engages as a result of lateral expansion of the concrete section under axial loading. As the member's axial load increases the corresponding lateral strain also increases causing the transverse reinforcement to develop tensile 
hoop stress balanced by a uniform radial pressure reacting against the concrete expansion $[16]$.

In 1929, using a continuous steel spiral as passive confinement, Richart et al. [26] found that the $k_{1}$ and $k_{2}$ coefficients had the same values as the coefficients found under active confinement. The continuity between the active and passive $k_{1}$ and $k_{2}$ coefficients was found to be contingent on the passive confinement spirals being spaced relatively close together. Other values for the $k_{1}$ coefficient were found by Balmer [5] and Talbot [31] to vary between 4.5 - 7.0 and $2.8-4.0$, respectively. In 1972, Newman and Newman [21] found that confinement efficiency decreases as confining pressure increases and is accounted for by a nonlinear relationship.

Since passive confinement can be as effective as active confinement and does not require the additional effort of prestressing, it is more commonly used in the construction industry. In addition, in 1996 active FRP confinement wraps installed on two columns near Los Angeles were found to have fractured. The fracture occurred roughly three years after the wraps were installed and almost one year after the Northridge earthquake. After conducting an analysis of the columns, it was concluded that the most likely reason for the confinement failures was due to stressing the wraps beyond their creep rupture strength during prestressing [13]. To avoid creep rupture and due to ease of installation, passive confinement is most common, and is thus the focus of study in this paper. 


\section{$2.2 \quad$ Stress-Strain Relationships}

Every material and/or combination of materials that forms composites has its own unique stress-strain relationships, which can be elastic, inelastic, or a combination of the two. In this paper several stress-strain relationship models for passively confined concrete are presented. Ultimately, one of the these confinement models is used for this study to conduct Moment-Curvature and Axial Force-Moment Interaction analyses.

Concrete is a non-homogeneous material prone to cracking. As a result, concrete's stress-strain relationship is highly non-linear [9]. Unconfined concrete and confined concrete have very different stress-strain relationships. Since concrete is weak in tension, loading induces the development of micro-cracks throughout the concrete cross section, growing in the unconfined concrete, until the strength of the section is greatly reduced. This causes failure in a very brittle fashion. However, with the addition of confinement around the concrete section, the micro-cracks, though still present, can be restrained and prevented from growing and causing failure at small compressive strains. The addition of confinement around reinforced concrete cross sections can change the concrete's stress-strain behavior from relatively weak and brittle to strong and ductile. The added strength and ductility found in confined concrete is due to the development of a triaxial stress field and the containment of the concrete after cracking [9].

To date, the most common form of transverse reinforcement in reinforced concrete columns has been steel spirals or hoops. Numerous studies outlining the effect of confinement on concrete strength and deformation have been conducted regarding both steel spirals and hoops, as well as rectilinear ties and concrete-filled steel tubular columns [16]. 
However, most of the stress strain models developed were only applicable for either circular or rectangular columns [24]. Mander et al. [17] used an energy balance approach along with effectiveness coefficients to unify the confinement model for both circular and rectangular transverse reinforcement at various spacing. Mander's model was based on the equation suggested by Popovics in 1973 [23] and has gained wide acceptance in the Civil Engineering field. The Mander confined concrete stress-strain model for a circular column with hoop transverse reinforcement, monotonic loading, and slow strain rate is shown in the following set of equations:

$$
f_{c}=\frac{f_{c c}^{\prime} x r}{r-1+x^{r}}
$$

where $f_{c}$ is the longitudinal compressive stress and $f_{c c}^{\prime}$ is the compressive strength of the confined concrete.

$$
x=\frac{\varepsilon_{c}}{\varepsilon_{c c}}
$$

where $\varepsilon_{c}$ represents longitudinal compressive strain.

$$
\varepsilon_{c c}=\varepsilon_{c o}\left[1+5\left(\frac{f_{c c}^{\prime}}{f_{c o}^{\prime}}-1\right)\right]
$$

where $f_{c o}^{\prime}$ and $\varepsilon_{c o}$ are the unconfined concrete strength and corresponding strain, respectively (generally $\varepsilon_{c o}$ can be assumed to be $0.002[25]$ ).

$$
r=\frac{E_{c}}{E_{c}-E_{s e c}}
$$


where

$$
E_{c}=57,000 \sqrt{f_{c o}^{\prime}}(\mathrm{psi})
$$

is the design tangent modulus of elasticity of the unconfined concrete from ACI 318-05 Chapter 8 Section 8.5.1.

$$
\begin{gathered}
E_{s e c}=\frac{f_{c c}^{\prime}}{\varepsilon_{c c}} \\
f_{c c}^{\prime}=f_{c o}^{\prime}\left(-1.254+2.254 \sqrt{1+\frac{7.94 f_{l}^{\prime}}{f_{c o}^{\prime}}}-2 \frac{f_{l}^{\prime}}{f_{c o}^{\prime}}\right) \\
f_{l}^{\prime}=\frac{2 k_{e} f_{y h} A_{s p}}{s d_{s}}
\end{gathered}
$$

where $f_{l}^{\prime}$ is the effective lateral confining stress, $k_{e}$ is the confinement effectiveness coefficient, $f_{y h}$ is the yield strength of the transverse reinforcement, $A_{s p}$ is the area of transverse reinforcement bar, $s$ is the center to center spacing or pitch of spiral or circular hoop, and $d_{s}$ is the diameter of spiral between bar centers.

$$
k_{e}=\frac{\left(1-\frac{s^{\prime}}{2 d_{s}}\right)^{2}}{1-\rho_{c c}}
$$

where $s^{\prime}$ represents the clear vertical spacing between hoops and $\rho_{c c}$ is the ratio of the longitudinal reinforcement area to the section's core concrete area. 
Below are several parameter relationships for reinforced concrete circular cross sections which Mander et al. [17] referenced to increase or decrease the confinement effectiveness on concrete dependent on their quantities.

- The transverse reinforcement spacing $(s)$ has an inverse relationship with the confinement effectiveness. Cross sections with large transverse reinforcement spacing have a large region of ineffectively confined concrete between each transverse bar, reducing the overall confinement effectiveness. Cross sections with small transverse reinforcement spacing have small regions of ineffectively confined concrete, increasing the cross section's overall confinement effectiveness.

- Well distributed longitudinal bars around the cross section $\left(k_{e}\right)$ increase the cross section's confinement effectiveness.

- Volume of transverse reinforcement has a direct correlation with the cross section's confinement effectiveness. Large transverse reinforcement volumes will increase a cross section's confinement effectiveness.

- Yield strength of transverse reinforcement has a direct correlation with the cross section's confinement effectiveness. High yield strength increases a cross section's confinement effectiveness.

\subsection{FRP Confinement and Stress-Strain Properties}

Though steel is the most common form of transverse reinforcement, in an effort to increase seismic performance of existing structures through retrofitting and new structures 
through design and detailing, other materials have and are being studied as possible supplements and/or alternatives to steel confinement. In 1978, Kurt [14] filled commercially available plastic pipes (PVS or ABS) with concrete. The test showed only a small increase in concrete strength from the plastic pipe confinement. The confined concrete in Kurt's experiment did not show a significant increase in strength because the plastics used were not relatively strong themselves, and thus, did not provide enough confinement of the core concrete to increase its strength significantly. FRP composites, unlike PVC and ABS, are much stronger than plastics and have been gaining popularity in their use as transverse reinforcement.

FRP composites use high-strength fibers directionally oriented in multiple layers and impregnated in a polymeric resin matrix of lower strength. The fibers are loaded in tension along their longitudinal axis while the matrix enables the load to be transferred between fibers and protects the fibers from environmental effects. The most common fiber types used are carbon, aramid, and glass. The fibers have an approximate ultimate strain range of $0.2 \%$ for Ultra-High-Modulus Carbon to $5.4 \%$ for S-Glass. They are linear elastic up to failure (no yielding), have an ultimate strength range of $200 \mathrm{ksi}$ for Ultra-High-Modulus Carbon to 900 ksi for Ultra-High-Strength Carbon, and have an elastic modulus range of 10,000 ksi for E-Glass and General Purpose Aramid to 100,000 ksi for Ultra-High-Modulus Carbon $(1 \mathrm{MPa}=0.145 \mathrm{ksi})[2]$. The mechanical properties of the FRP composites are dependent on the constituents and their proportions [16].

FRP's used as confinement for reinforced concrete cross sections have three typical configurations: filaments, wraps, and tubes. Filament winding is done by wrapping fibers 
impregnated with resin around a column. Wraps are sheets of fibers that are laminated around the column in multiple layers. The fibers in the sheets can be woven in different patterns and/or the sheets themselves can be oriented such that the longitudinal direction of the fibers are best suited to carry the load demands. FRP tubes are formed in a factory to a specific size with a wall thickness, strength, and stiffness according to the design specifications. The tubes are brought to a job site and used as permanent formwork for columns, limiting their use to new construction. Filaments and wraps, like steel, can be used in either new construction or retrofitting of existing structures.

FRP and steel, however, have different material properties and therefore the existing and accepted stress-strain models for steel confined concrete do not necessarily apply to FRP confined concrete. Steel has three distinct regions in its stress-strain relationship: linear elastic region, yielding plateau, and strain hardening region. The three regions can be seen in Figure 2.1. Steel possesses linear elastic properties up to its yield strength at which point its stress-strain relationship plateaus. Once yielding has begun, the steel confinement does not provide any additional confinement pressure though both the column's axial load and subsequent lateral dilation are increasing. The yield plateau ends when the cross section's lateral dilation is large enough that the transverse steel reaches its strain hardening point. Once the transverse steel reaches its strain hardening point its stiffness is increased providing additional confining pressure. Throughout strain hardening, steel confinement stiffness decreases nonlinearly until failure.

Unlike steel, FRP materials remain linear elastic up to failure. In Figure 2.1 the three regions of the steel stress-strain relationship as well as the linear elastic behavior of 


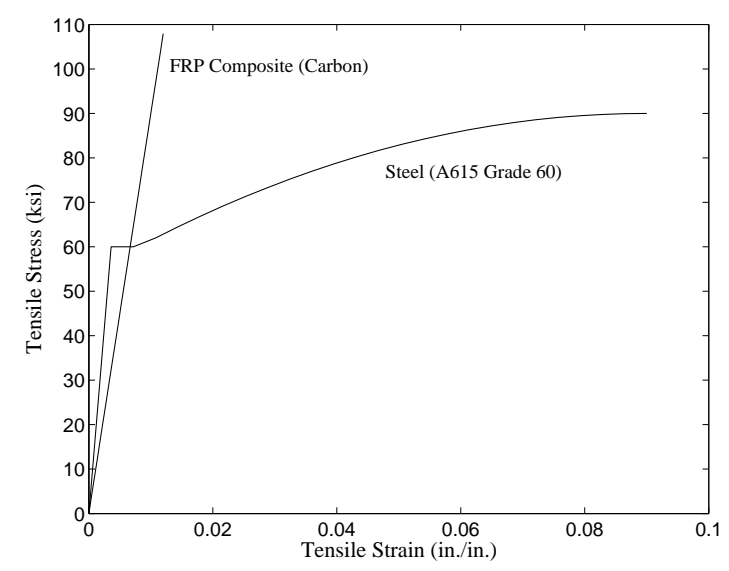

FIGURE 2.1: Steel and FRP Stress-Strain Relationships

the FRP material are depicted. Another difference between steel and FRP confinement materials is their stiffnesses. With the exception of high strength Carbon, steel is much stiffer than FRP. The different stress-strain relationships and stiffnesses of steel and FRP greatly change the composite stress-strain relationship of confined concrete.

Figure 2.2 superimposes stress-strain plots for unconfined concrete, steel confined concrete, and FRP confined concrete produced from the Mander Unconfined [17], Mander Confined [17], and Samaan Confined [29] concrete models, respectively. These plots show that up to the unconfined concrete strength of $4.00 \mathrm{ksi}$, both steel confined concrete and FRP confined concrete behave similarly. At the point where the concrete's unconfined strength is reached, the concrete begins to lose strength and dilate causing the confinement to engage. Once the confinement engages, its stiffness controls the stress-strain relationship. Notice in Figure 2.2, at the unconfined concrete strength, the steel confined concrete has greater stiffness than the FRP confined concrete; however, at a concrete stress of just over $5.00 \mathrm{ksi}$ the steel begins to yield and the composite strength of the steel confined concrete 


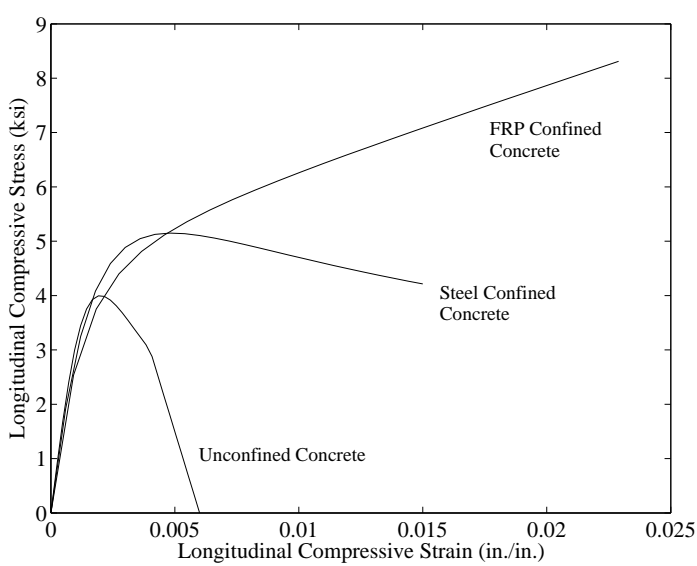

FIGURE 2.2: Concrete Stress-Strain Relationships

begins to decrease until failure. It can also be seen that at the same point the steel begins to yield, the FRP confinement fully engages and controls the stress-strain relationship resulting in a bilinear stress-strain curve with a mild transition zone. Another difference between steel confinement and FRP confinement is that steel only forms a mechanical bond with the core concrete while the FRP confinement can form both a mechanical and chemical bond. However, tests have shown that FRP confinement chemically bonded to the concrete members does not vary significantly from FRP confinement mechanically bonded [18]. Thus the type of bond between the FRP jacket and the concrete core is not considered as a parameter in this study.

To calculate the confinement pressure applied to the core concrete by the FRP confining mechanism, strain compatibility must be assumed between the FRP confining mechanism and the concrete surface. That is, the lateral strain of the confined concrete core must equal the strain of the FRP confining mechanism. As the confining mechanism is strained a hoop stress acting against further dilation is induced. Figure 2.3 demonstrates 


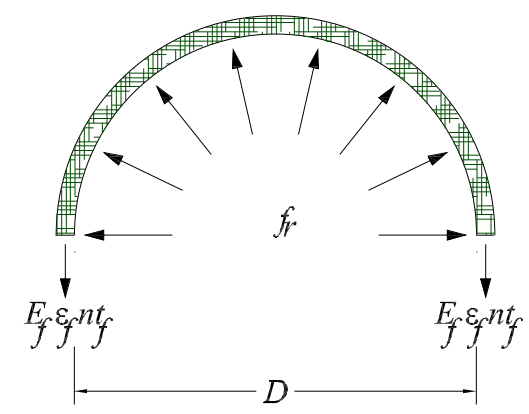

FIGURE 2.3: FRP Confinement Free-Body Diagram

how the outward pressure from the lateral dilation of the core concrete is restrained and in equilibrium with the hoop stress of the confining mechanism.

Summing the forces applied to the cross section by the FRP confinement in equilibrium results in the following equation:

$$
E_{r}=\frac{2 E_{f} n t_{f}}{D}
$$

where $E_{r}$ represents the confinement modulus, $E_{f}$ is the FRP modulus, $n$ is the number of FRP layers, $t_{f}$ is the FRP layer thickness, and $D$ is the diameter of column. Then the confinement pressure $f_{r}$ is calculated using the FRP strain $\left(\varepsilon_{f}\right)$ and the FRP stress $\left(f_{f}\right)$ for corresponding $\varepsilon_{f}$ in Equation 2.13. 


$$
f_{r}=E_{r} \varepsilon_{f}=\frac{2 f_{f} n t_{f}}{D}
$$

The confinement modulus $\left(E_{r}\right)$ can be of significant importance to ductility and, in turn, seismic design. Zhang [32] found that the added confinement from one layer of an FRP wrap can change a column's failure mode from a shear (brittle) to flexure (ductile). However, the one layer of FRP did not provided result in a large enough $E_{r}$ to significantly increase the column's ductility. In the same study, the column wrapped with four FRP layers and thus having four times the $E_{r}$ was found to have rich ductility. This result demonstrates that ductility and the desired elastoplastic behavior of a column under large ground motions is strongly affected by $E_{r}$.

The ultimate confinement pressure is a function of the confinement modulus and the pressure FRP applies to a concrete cross section when its hoop strain reaches its ultimate strain. Once the ultimate strain is achieved, the FRP confinement will rupture causing the column to also fail in a very brittle manner. The ultimate confinement pressure $\left(f_{r u}\right)$ can be calculated by substituting the ultimate FRP stress $\left(f_{f u}\right)$ and its corresponding FRP ultimate strain $\left(\varepsilon_{f u}\right)$ into 2.13 , resulting in 2.14 below.

$$
f_{r u}=E_{r} \varepsilon_{f u}=\frac{2 f_{f u} n t_{f}}{D}
$$

where $f_{f u}=E_{f} \varepsilon_{f u}$ and $E_{f}$ is the FRP modulus of elasticity. 


\subsection{FRP Confinement Models}

Three models for concrete passively confined using FRP composites are presented below. The models are selected to demonstrate the various methodologies researchers use to develop their confinement models. The first model, "Steel Based Confinement Model", is based on existing steel confinement models and modified to accommodate FRP properties. The second model, "Empirical Confinement Model", uses a bilinear curve with a transition zone utilizing four parameters to calibrate the stress-strain behavior. The last model, "Bilinear Confinement Model", has two main characteristics: (1) a constitutive relationship consisting of only two lines, and (2) simple equations to represent the two lines [28].

\subsubsection{Saadatmanesh et al. (1994) Steel Based Confinement Model}

Saadatmanesh et al. [27] based their FRP confinement model on the well established steel confinement model proposed by Mander [17]. Like Mander's model, this FRP confinement model is based on energy balance and an ultimate strength surface. The energy balance is calculated by equating the added ductility of the confined concrete to the energy stored in the confining device [16]. Saadatmanesh's FRP confinement model for circular sections can be calculated using the following equation:

$$
\frac{f_{c c}^{\prime}}{f_{c o}^{\prime}}=2.254 \sqrt{1+7.94 \frac{p}{f_{c o}^{\prime}}}-2 \frac{p}{f_{c o}^{\prime}}-1.254
$$

Where $f_{c c}^{\prime}$ is the ultimate confined concrete strength, $f_{c o}^{\prime}$ is the unconfined concrete strength, and $p$ is the ultimate confinement pressure shown as $f_{r u}$ in Equation 2.14. Once the ultimate confinement strength is calculated the entire stress-strain curve can be equated 
using Equations 2.3 through 2.8 developed by Mander.

Saadatmanesh et al. used this model for a parametric study in their research and did not compare this model with experimental results. However, other authors have shown that Saadatmanesh's model compares reasonably well with experimental results [16].

\subsubsection{Samaan et al. (1998) Empirical Confinement Model}

Samaan's FRP confinement model is an empirical bilinear curve with a transition zone located at the strain associated with unconfined concrete's peak stress. The model is based on the correlation between the reinforced concrete cross section's lateral dilation and the stiffness of the restraining device. The parameters of the model are set from the material properties of the core concrete and the FRP confining device. The equations for the bilinear response are the following:

$$
f_{c}=\frac{\left(E_{1}-E_{2}\right) \varepsilon_{c}}{\left(1+\left(\frac{\left(E_{1}-E_{2}\right) \varepsilon_{c}}{f_{o}}\right)^{n}\right)^{1 / n}}+E_{2} \varepsilon_{c}
$$

where $f_{c}$ and $\varepsilon_{c}$ represent the axial stress and strain, respectively; and

$$
\begin{gathered}
E_{1}=47,586 \sqrt{f_{c}^{\prime}} \\
E_{2}=52.411 f_{c}^{\prime 0.2}+1.3456 \frac{E_{f} n_{f} t_{f}}{D}(\mathrm{ksi})
\end{gathered}
$$

where $E_{1}$ is the elastic modulus of the unconfined concrete and the first slope of the curve. $E_{2}$ is the second slope of the curve, dependent on the stiffness of the confining device. $f_{c}^{\prime}$ is the unconfined concrete strength, $E_{f}$ is the elastic modulus of the FRP confining 
device, $n_{f}$ is the number of FRP layers, $t_{f}$ is the thickness of one FRP layer, and $D$ is the cross section diameter of the concrete. This model is not sensitive to the curve-shaped parameter $(n)$ and therefore it is assigned a constant value of 1.5. It is worth noting that Equation 2.17 is used to calculate the unconfined concrete elastic modulus in lieu of the more common ACI 318-05 Chapter 8 Section 8.5.1 equation as it has been shown to over estimate the unconfined concrete's elastic modulus [3]. The ultimate compressive strength of the confined concrete $\left(f_{c u}^{\prime}\right)$ can be calculated by the following equation:

$$
f_{c u}^{\prime}=f_{c}^{\prime}+k_{1} f_{r u}
$$

where $k_{1}$ is the coefficient of effectiveness and can be seen in Equation 2.20, and $f_{r u}$ is the confinement pressure and can be calculated using Equation 2.13.

$$
k_{1}=3.38 f_{r u}^{-0.3}(\mathrm{ksi})
$$

Substituting Equation 2.20 into Equation 2.19 the ultimated confined concrete strength can be calculated using the following:

$$
f_{c u}^{\prime}=f_{c}^{\prime}+3.83 f_{r u}^{0.7}(\mathrm{ksi})
$$

The equation for $f_{o}$ below calculates the stress at the bilinear interception point.

$$
f_{o}=0.872 f_{c}^{\prime}+0.371 f_{r u}+0.908(\mathrm{ksi})
$$


By using the the ultimate confined concrete strength, the intercept strength, and the slope of the second line the ultimate confined concrete strain can be calculated below:

$$
\varepsilon_{c u}=\frac{f_{c u}^{\prime}-f_{o}}{E_{2}}
$$

Samaan et al. [29] compared the analytical results of their model to their experimental results from the same study and the results of several other similar studies. The model was found to achieve a good correlation.

\subsubsection{Saiidi et al. (2005) Bilinear Confinement Model}

Saiidi's FRP confinement model, like Samaan's, is an empirical model with a bilinear curve; however, Saiidi's model excludes a transition zone. Instead, the model only requires two points: the break point and the ultimate point. The break point occurs where each of the bilinear curves intersect. The stress at the point of intersection is calculated by Equation 2.24. The ultimate point corresponds to the ultimate stress of the confined concrete, calculated by Equation 2.27. The goal of this study was to produce a model accurate enough for Moment-Curvature analyses while being simple in nature. The break point and the ultimate point can be calculated using the equations that follow.

$$
f_{c o}^{\prime}=f_{c}^{\prime}+0.003 \rho_{c f} E_{j}
$$

where $f_{c o}^{\prime}$ represents stress at the break point corresponding to the axial strain $\left(\varepsilon_{c}\right)$ of $0.002, f_{c}^{\prime}$ is the unconfined concrete strength, $\rho_{c f}$ is the volumetric ratio of the FRP 
jacket, and $E_{j}$ is the elastic modulus of the FRP jacket. The confinement pressure $\left(f_{r}\right)$ is found from Equation 2.25.

$$
f_{r}=\frac{2 E_{j} \varepsilon_{j} t_{j}}{D}
$$

where $\varepsilon_{j}$ is the strain in the FRP jacket, $t_{j}$ is the thickness of the FRP jacket, and $D$ is the diameter of the column. The next two equations provide the ultimate strain $\left(\varepsilon_{c u}\right)$ and the ultimate stress $\left(f_{c u}^{\prime}\right)$ in order to calculate the ultimate point of the model. In Equation 2.26, $\varepsilon_{f}$ is set equal to $50 \%$ of the ultimate FRP fiber failure strain (effective failure strain); the value of $f_{r}$ from Equation 2.25 is also calculated using $\varepsilon_{j}=50 \%$ of the ultimate FRP fiber strain to be used in both Equation 2.26 and Equation 2.27.

$$
\begin{gathered}
\varepsilon_{c u}=\frac{\varepsilon_{f}}{\left(0.1-0.25 \ln \left(\frac{f_{r}}{f_{c}^{\prime}}\right)\right)} \\
f_{c u}^{\prime}=f_{c}^{\prime}+3.5 f_{r}^{0.7}(\mathrm{ksi})
\end{gathered}
$$

Saiidi et al. [28] found that their simplified bilinear model was able to estimate the Moment-Curvature relationships of typical columns with reasonable accuracy.

\subsection{Selected Confinement Model}

While Saadatmanesh's steel based confinement model has been shown to predict the stress-strain relationship of FRP confined concrete with reasonable accuracy [16], other studies have shown that models based on steel may not be conservative enough due to 
the inherent differences between FRP composites and steel [29]. Mander's energy balance approach developed for steel confined concrete neglects lateral strain energy stored in the concrete which in FRP confined concrete, accounts for close to $80 \%$ of the total energy [12].

Saiidi et al. developed their confinement model for Moment-Curvature relations on the principle that Moment-Curvature relations are generally insensitive to slight variations in concrete constitutive relations. Therefore simple models should yield similar results to complex models [28]. Thus, their model was able to predict the Moment-Curvature relations of typical columns with reasonable accuracy.

Samaan's confinement model has good correlation with the experimental data set used in the development of the model, three other data sets used to validate the model, and numerous other data sets compiled for a comparative study [29], [16]. However, when compared to similar models, Samaan's model overestimates the ultimate strain and produces non-conservative results [28].

Samaan's confinement model, nevertheless, is the model used in Chapter 3 to conduct Moment-Curvature and PM Interaction analyses. This model is selected because of its good correlation over a wide range of data sets along with its use of a conservative unconfined concrete elastic modulus (first slope of its bilinear curve). However, due to its tendency to overestimate the ultimate FRP strain due to typical premature fiber failure, an effective ultimate FRP strain similar to the one used by Saiidi et al. is advised. Therefore, this study follows the recommendations of $A C I$ 440.2R-08 and reduces the ultimate FRP strain by multiplying it by a strain efficiency factor, $\kappa_{e}[2]$. The ultimate effective FRP strain can then be calculated by Equation 2.28 and the ultimate effective FRP tensile stress 
can be calculated by Equation 2.29 .

$$
\begin{gathered}
\varepsilon_{f e}=\kappa_{e} \varepsilon_{f u} \\
f_{f e}=\kappa_{e} E_{f} \varepsilon_{f u}
\end{gathered}
$$

In addition, $A C I 440.2 R-08$ states that a strain efficiency factor $\kappa_{e}$ of 0.55 should be used [2]. Now, Equation 2.14 used to calculate the ultimate confinement pressure $\left(f_{r u}\right)$ can be updated to calculate the effective ultimate confinement pressure $\left(f_{r e}\right)$ and is shown below:

$$
f_{r e}=E_{r} \varepsilon_{f e}=\frac{2 f_{f e} n t_{f}}{D}
$$

This study utilizes Samaan's confinement model in conjunction with the $A C I$ 440.2R-08 recommended effective ultimate FRP strain to achieve accurate yet slightly more conservative results. 


\section{CHAPTER 3}

\section{Moment-Curvature and Axial}

\section{Force-Moment Interaction}

\subsection{Introduction}

Strong ground motions from a seismic event may cause inelastic displacements and axial loads to vary in the plastic hinge regions of columns. For good seismic performance, plastic hinges must have adequate rotational capacity to achieve required seismic displacement demands; enough ductility to withstand several inelastic displacement cycles without significant loss of flexural strength; and a reliable flexural overstrength so accommodations can be made in non-ductile members so they remain elastic under seismic loading. Moment-Curvature and Axial Force-Moment Interaction diagrams are good tools to assess the rotational capacity, ductility, flexural strength, and flexural overstrength of cross sections needed to properly design for seismic events. 
In this Chapter, both the Moment-Curvature and Axial Force-Moment Interaction analysis methodologies are reviewed and software to conduct such analyses is developed. The developed software will be used in Chapter 4 to assess the seismic performance of FRP confined cross sections through a comparative analysis between steel confined and FRP confined cross sections. Additionally, this software will perform a series of parameter studies on typical design variables for FRP confined cross sections in order to better understand the behaviors of this configuration as they relate to seismic design.

The following is a list of necessary assumptions used in the development of the Moment-Curvature and Axial Force-Moment Interaction software:

- Plane sections remain plane

- $\theta \simeq 0, \tan \theta=\theta$ (small angle theory)

- FRP confined concrete sections fail when:

1. The confined core concrete crushes in compression due to jacket rupture at its effective ultimate fiber strain, and/or

2. The longitudinal rebar ruptures

- Steel confined concrete sections fail when:

1. The confined core concrete crushes due to loss of confinement, and/or

2. The longitudinal rebar ruptures

- Concrete tensile strength is assumed to be zero, consistent with seismic design

- The post crushing concrete strength of the core concrete is taken to be zero 
- Unconfined cover concrete begins to crush at a compressive strain of 0.004 and completely spalls at a compressive strain of 0.006

- The FRP confining jacket of the confined concrete has a much greater transverse stiffness than longitudinal stiffness, and therefore only acts as transverse reinforcement. (This is to ensure the FRP jacket does not absorb any axial load which can cause premature buckling.)

\subsection{Moment-Curvature Analysis Methodology}

Moment-Curvature analyses require stress-strain material models for each cross section material. The models are used to find the stress in each of the different materials in a cross section at a given strain. The following are the stress-strain material models used by the developed software in this study:

- Mander et al. [17] steel confined concrete model shown in Equations 2.3 through 2.11

- Mander et al. [17] unconfined concrete model

- Priestley [7] bilinear steel model with parabolic strain hardening

- Samaan et al. [29] FRP confined reinforced concrete model for circular cross sections.

Once the models for a cross section's different materials are selected, the next step is to discretize that cross section. Since this study focuses on uni-axial bending, the cross section is divided up into a series of layers discretized parallel to the axis of bending. The number of layers used is a function of balancing computation time with accuracy. The 
more layers used, the more accurate and time consuming the Moment-Curvature analysis. To determine an adequate layer gradation, a sensitivity analysis is conducted on a steel confined reinforced concrete cross section. The properties of the cross section used in this sensitivity analysis can be found in Table 3.1. Figure 3.1 shows seven Moment-Curvature analyses conducted on the cross section under identical loading conditions but with varying numbers of layers. In the first and second Moment-Curvature analyses the cross section is discretized into five and 10 layers, respectively. Each additional Moment-Curvature analysis increases the number of layers in the cross section by 10 until reaching the final analysis with 70 layers. It is demonstrated in Figure 3.1 that the cross sections with only five or 10 layers produce inconsistent and non-conservative results; while the cross sections with 20 or more layers converge to a uniform envelope. Figure 3.2 shows the percent variance between each consecutive Moment-Curvature analysis to demonstrate how the data from these analyses converges as the number of layers increase. It also demonstrates that the variance between 40 layers and 30 layers is less than $0.5 \%$ and that increasing the number of layers beyond 40 does not greatly reduce the variance. While increasing the number of layers beyond 40 does not significantly reduce variance, it does significantly increase computation time. Thus, in this study the cross sections are discretized into 40 layers.

After the cross section is discretized, each layer is assigned a material type linking that layer's material to its corresponding material model. In addition, each layer has an area $\left(A_{i}\right)$ and centroid location $\left(\bar{y}_{i}\right)$. The centroid location is defined relative to the centroid of the cross section which is set at the $(0,0)$ coordinate. This is done to avoid an eccentric loading condition since the axial load is applied to the $(0,0)$ coordinate. Next, all the 
TABLE 3.1: Discretization Sensitivity Study Cross Section Configuration

\begin{tabular}{|l|l|}
\hline Section Property & Quantity \\
\hline Section Diameter: & $24.00 \mathrm{in}$ \\
\hline Gross Section Area: & $452.39 \mathrm{sqin}$ \\
\hline Unconfined Concrete strength: & $4.00 \mathrm{ksi}$ \\
\hline Unconfined Concrete Elastic Modulus: & $57.00 \sqrt{1000 f_{c}^{\prime}} \mathrm{ksi}$ \\
\hline Number of Longitudinal Bars: & 8 \\
\hline Longitudinal Bar Size: & $\# 7$ \\
\hline Longitudinal Bar Area: & $4.80 \mathrm{sqin}$ \\
\hline Longitudinal Reinforcing Ratio: & 0.011 \\
\hline Reinforcing Steel Yield Stress: & $60.00 \mathrm{ksi}$ \\
\hline Reinforcing Steel Fracture Stress: & $90.00 \mathrm{ksi}$ \\
\hline Reinforcing Steel Strain at Strain Hardening: & 0.0080 \\
\hline Reinforcing Steel Failure Strain: & 0.090 \\
\hline Reinforcing Steel Elastic Modulus: & $29,000.00 \mathrm{ksi}$ \\
\hline Applied Axial Load: & $200 \mathrm{kips}$ \\
\hline Type of Transverse Reinforcement: & Steel Hoop \\
\hline Transverse Reinforcing Bar Size: & $\# 4$ \\
\hline Spacing of Transverse Reinforcement: & $4.00 \mathrm{in}$ \\
\hline Cover Concrete Thickness: & 1.50 in \\
\hline
\end{tabular}

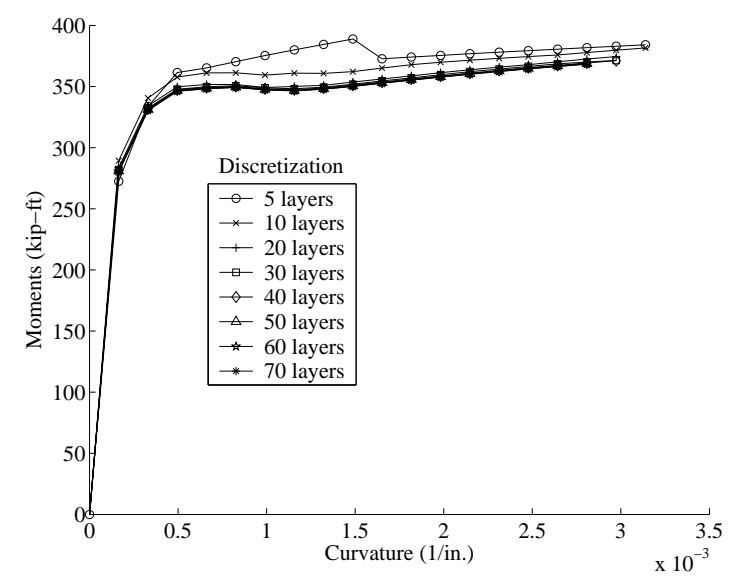

FIGURE 3.1: Discretization Sensitivity Analysis 


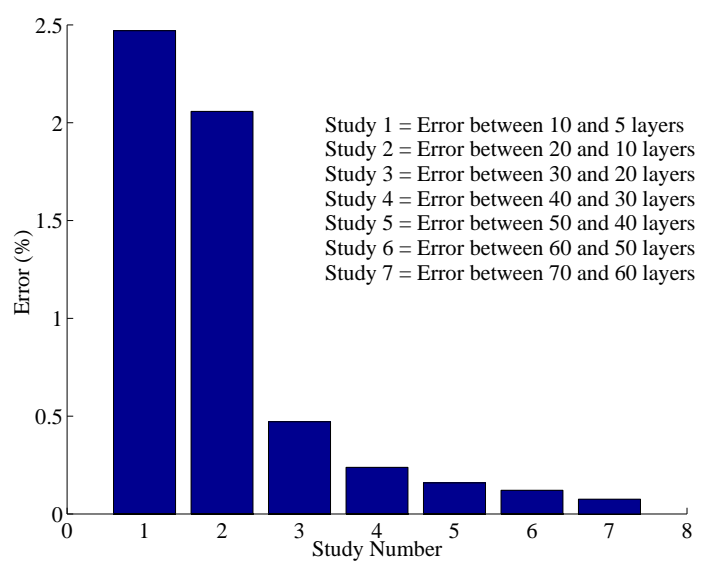

FIGURE 3.2: Discretization Sensitivity Error Analysis

different material layers are mapped to their associated coordinate positions. Overlapping material layers, such as rebar and concrete, must have their areas algebraically adjusted so that two different materials do not exist in the same space. The developed software stores each layer's material type and corresponding stress-strain model, area, and centroid location in a matrix to be utilized during the analysis.

Figure 3.3 shows the cross section used in the sensitivity study discretized with the material types of the layers mapped. Notice that since this is a steel hoop confined cross section it has three material types: unconfined concrete, steel confined concrete, and steel longitudinal reinforcement. Due to the assumption that the FRP confinement only acts in the transverse direction, FRP confined reinforced concrete cross sections have only two discretized materials: FRP confined concrete and longitudinal rebar.

After discretization and material identification, the applied axial load $(P)$ acting on the cross section and the analysis method are selected. In the developed software the applied axial load can be specified to match a specific loading condition or a series of 


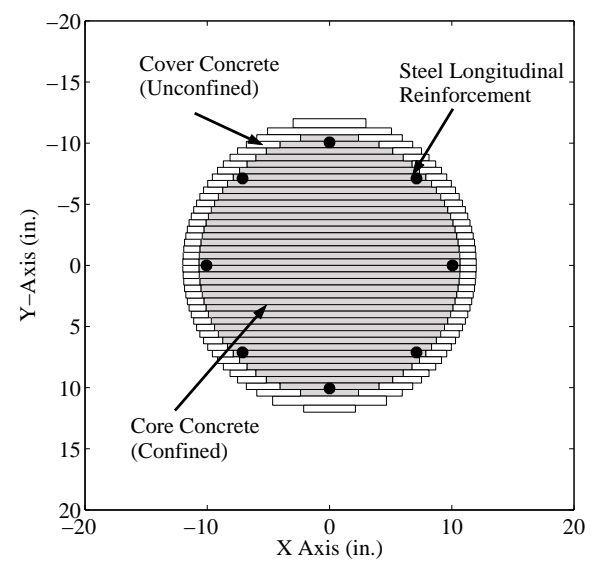

FIGURE 3.3: Cross Section Materials

axial loads can be specified to conduct a sequence of Moment-Curvature analyses. The two analytical methods generally used are force control and displacement control. Both the force control and displacement control methods produce results that are consistent with each other for axisymetric cross sections; however, the force control method has a problem with convergence when there are severe discontinuities defined within the material models [8]. Thus, the software developed as part of this study uses the displacement control method.

The displacement control analysis is conducted by imposing incremental curvatures $\left(\phi_{j}\right)$ onto the cross section until the first material limit state is reached as described in section 3.1. Next, the strain $\left(\varepsilon_{i j}\right)$ at each layer's centroid is calculated using the imposed $\phi_{j}$, each layer's $\bar{y}_{i}$, an assumed geometric centroid strain $\left(\varepsilon_{j o}\right)$, and strain compatibility through the following equation:

$$
\varepsilon_{i j}=\varepsilon_{j o}-\phi_{j} \bar{y}_{i}
$$




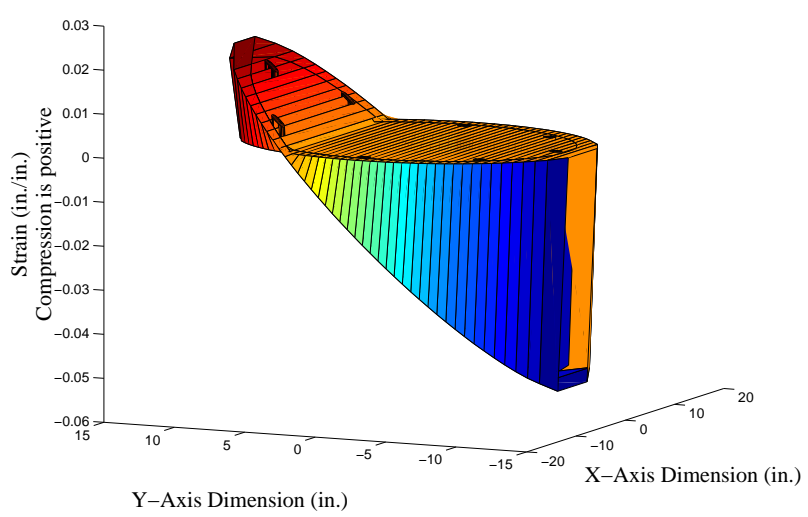

FIGURE 3.4: Cross Section Strain Distribution

Figure 3.4 shows the strain distribution in the steel hoop confined reinforced concrete cross section used in the discretization sensitivity study at failure due to concrete crushing.

Each layer's centroid strain is substituted into the material model to calculate its stress:

$$
\sigma_{i j}=f_{k}\left(\varepsilon_{i j}\right)
$$

where $k$ denotes the constitutive relation consistent with the material model of the layer. Figure 3.5 shows the stress throughout the steel hoop confined reinforced concrete cross section used in the discretization sensitivity study at failure due to concrete crushing.

Each layer's reaction force $\left(F_{i j}\right)$ for the applied $\phi_{j}$ can then be calculated by Equation 3.3: 


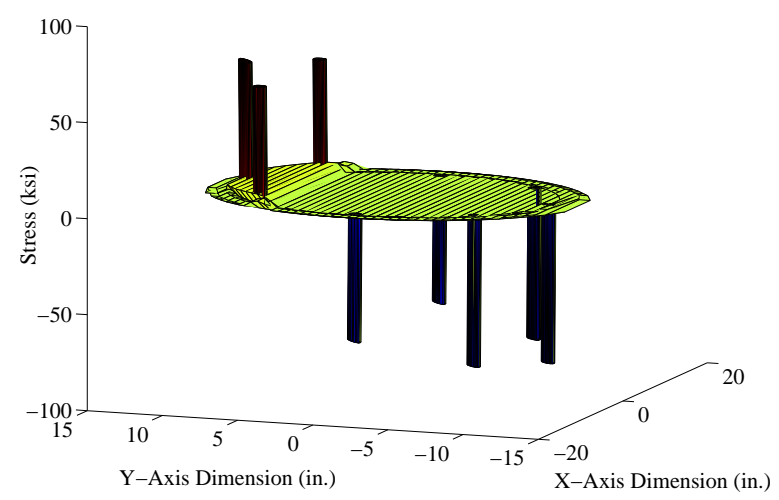

FIGURE 3.5: Cross Section Stress Distribution

$$
F_{i j}=A_{i} \sigma_{i j}
$$

With each layer's reaction force known, an iteration process is conducted to satisfy equilibrium by varying the cross section's centroid strain $\left(\varepsilon_{j o}\right)$ at the specified curvature until the resisting axial load balances with the applied axial load for that step. The iteration is complete when Equation 3.4 is satisfied. Then with the axial loads in equilibrium the moment $\left(M_{j}\right)$ corresponding to $\phi_{j}$ is calculated using the following Equation 3.5 to produce the coordinate pairs of the Moment-Curvature diagram.

$$
\begin{gathered}
P_{j}=\sum_{i k} A_{i} f_{k}\left(\varepsilon_{j o}-\phi_{j} \bar{y}_{i}\right) \\
M_{j}=\sum_{i k}-\bar{y}_{i} A_{i} f_{k}\left(\varepsilon_{j o}-\phi_{j} \bar{y}_{i}\right)
\end{gathered}
$$


The force control analysis is conducted by imposing incremental moments $\left(M_{j}\right)$ onto the cross section until failure. For each applied moment increment there is a NewtonRaphson type iteration that uses the tangent cross section stiffness to sum each reaction force in every layer in the cross section until equilibrium is reached with the applied axial load [8]. Once equilibrium is achieved the associated curvature $\left(\phi_{j}\right)$ is logged and assigned to the applied moment to form a Moment-Curvature coordinate pair $\left(M_{j}, \phi_{j}\right)$. All the $(M, \phi)$ coordinate pairs up to the section's failure are plotted to produce the Moment-Curvature diagram.

\subsection{Axial Force-Moment Interaction Analysis Methodology}

An Axial Force-Moment Interaction diagram, also called a PM Interaction diagram, is a plot of a combination of axial load $(P)$ and corresponding moment $(M)$ coordinate pairs that when applied to the cross section cause first material failure. Similar to Moment-Curvature diagrams, Axial Force-Moment Interaction diagrams require accurate stress-strain models to produce reliable seismic assessments of plastic hinge cross section properties. Also similarly, discretization of the cross section into layers (fibers if biaxial bending is considered), and definition and mapping of layer properties is required. The software used in this study utilizes the same material models, cross section discretization, and layer properties for both PM Interaction diagrams and Moment-Curvature diagrams. Furthermore, one coordinate pair on a PM Interaction diagram corresponds to an applied axial load and correlating ultimate moment calculated from a Moment-Curvature analysis. Figures 3.6 and 3.7 demonstrate how a PM Interaction diagram is connected to a section's 


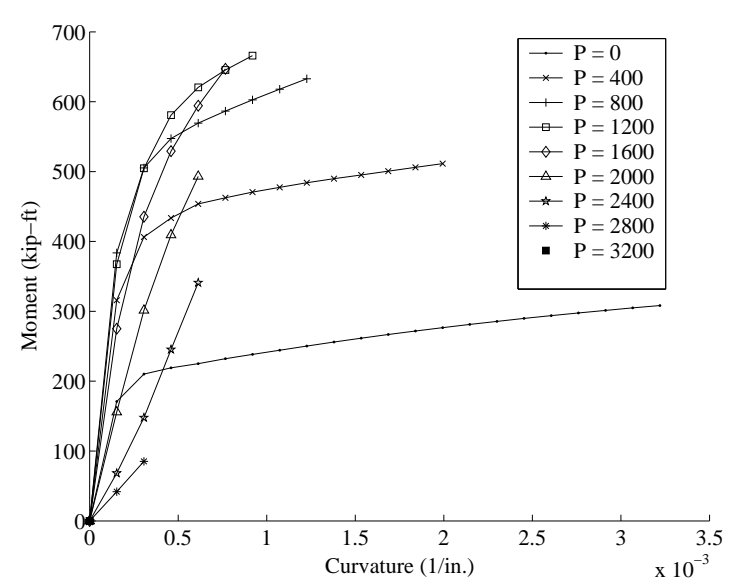

FIGURE 3.6: Influence of Axial Load on Moment-Curvature Relationship

Moment-Curvature properties. Figure 3.6 shows nine Moment-Curvature analyses conducted on the same cross section configuration at nine different loads. The last of the nine analyses appears on Figure 3.6 as a single point at the origin $(\phi=0, \mathrm{M}=0)$ because the applied axial load in this analysis was the cross section's Ultimate Axial Load. Therefore, any imposed curvature and resultant moment beyond this will cause failure. Figure 3.7 shows a PM Interaction diagram for the cross section comprised from the nine Moment-Curvature analyses. Each point on this Interaction curve corresponds to the axial load applied to the cross section and its correlating moment found at the Ultimate Curvature for each of the nine analyses shown in Figure 3.6.

However, PM Interaction diagrams may be constructed without using a series of Moment-Curvature analyses. Instead, PM Interaction diagrams can be constructed by focusing on critical material layers in a cross section and a specific centroid strain range. The critical layers in a cross section are the greatest distance from the geometric centroid of the cross section for each material type. The centroid strain range is established by 


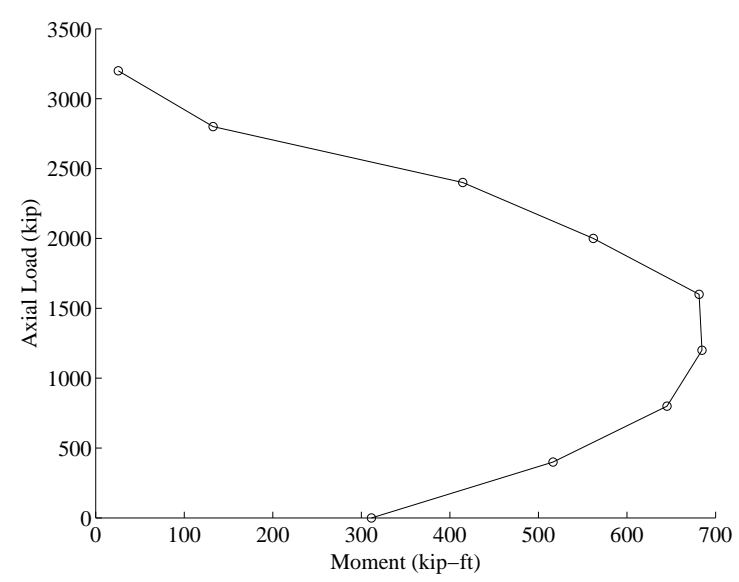

FIGURE 3.7: Ultimate Moment PM Interaction Curve

calculating the failure strains under pure tension and pure compression. This range can then be divided into the number of points on the PM Interaction diagram. At each centroid strain the minimum curvature corresponding to the first critical layer failure is found. Using this curvature for each centroid strain condition, the corresponding moments can be calculated and combined with the axial forces associated with the particular strain diagrams producing Axial Force-Moment coordinate pairs [8]. This method reduces computation time while producing accurate results necessary to make a reliable seismic assessment of plastic hinge cross section properties.

\subsection{Conclusion}

The methodology and development of software for both Moment-Curvature and PM Interaction diagrams necessary to make seismic assessments of plastic hinge cross section properties have been discussed in this Chapter. To ensure the accuracy of the developed software's Moment-Curvature and PM Interaction diagrams, a comparison between 
the developed software and XTRACT [7], a commercially available cross section analysis program, is conducted. It is found that the developed software produces the same results as XTRACT [7]. Thus, validating this software for use in the subsequent confinement comparison and parameter studies. The following Chapter utilizes the developed MomentCurvature and PM Interaction software to review the seismic performance of FRP confined cross sections by conducting a comparison between FRP and steel confinement, and by performing parameter studies on typical design variables. 


\section{CHAPTER 4}

\section{Confinement Comparison and}

\section{Parameter Study}

\subsection{Introduction}

In this Chapter, results of a comparative study between steel confined and FRP confined reinforced concrete are presented in addition to the results of a design variable parameter study on FRP confined reinforced concrete circular cross sections. The comparative study is conducted to determine if FRP materials can confine reinforced concrete as effectively as steel and to identify differences between the two confining systems that are critical when designing for seismic resistance. Parameter studies are presented to identify member displacement capacity, ductility, flexural strength, and flexural overstrength trends associated with each design variable so that FRP confined plastic hinges and surrounding elastic components can be designed for seismic requirements. 
Moment-Curvature analyses are used to determine the Yield Moment $\left(M_{y}\right)$, Yield Curvature $\left(\phi_{y}\right)$, Ultimate Moment $\left(M_{u}\right)$ and Ultimate Curvature $\left(\phi_{u}\right)$, which are then used to calculate the Section Plastic Curvature, Section Curvature Ductility, and Section Overstrength Factor of each cross section at Axial Load Ratios between 0.1 and 0.5. The Axial Load Ratio $\left(P_{r}\right)$, as defined in Equation 4.1, is the ratio between the cross section's Axial Load $(P)$ and the product of the cross section's unconfined concrete compressive strength $\left(f_{c}^{\prime}\right)$ and the gross cross section area $\left(A_{g}\right)$.

$$
P_{r}=\frac{P}{f_{c}^{\prime} A_{g}}
$$

The Section Plastic Curvature (Equation 4.2) is the difference between the cross section's $\phi_{u}$ and $\phi_{y}$, specific to an axial load. The Ultimate Curvature is the largest curvature that can be imposed on a cross section without causing failure at that specific axial load. The Yield Curvature is the curvature at first material yielding of the cross section. Therefore, the Section Plastic Curvature measures the inelastic rotational capacity of a cross section, having a major influence on the member displacement capacity.

$$
\phi_{p}=\phi_{u}-\phi_{y}
$$

The Section Curvature Ductility (Equation 4.3) is defined as the ratio between a cross section's $\phi_{u}$ and $\phi_{y}$, specific to an axial load. Thus, it measures the inelastic rotational capacity relative to the elastic yield rotation and, therefore, is directly related to member and structure ductility. Thus, provided the Section Overstrength Factor is greater than one, increasing Section Curvature Ductility increases member and structure ductility. 


$$
\mu_{\phi}=\frac{\phi_{u}}{\phi_{y}}
$$

The Section Overstrength Factor (Equation 4.4) measures $M_{u}$, defined as the moment corresponding to $\phi_{u}$, relative to $M_{y}$, defined as the moment corresponding to $\phi_{y}$, specific to an axial load. Thus, the Section Overstrength Factor measures the ability of a cross section to maintain flexural strength when inelastically loaded, directly relating to member and structure ductility.

$$
\Omega_{o}=\frac{M_{u}}{M_{y}}
$$

The Section Plastic Curvature, Section Curvature Ductility, and Section Overstrength Factor are directly related to member and structure displacement and ductility, measures of a structure's ability to sustain seismic inelastic deformation cycles without collapse or loss of strength. To demonstrate this relationship, the following shows how the member and structural displacement and ductility of a bridge supported by a single cantilever column on a spread footing are calculated using the section properties listed above. The member and structure displacement $\left(\Delta_{u}\right)$ is calculated using Equation 4.5:

$$
\Delta_{u}=\Delta_{y}+\Delta_{p}
$$

where $\Delta_{y}$ is the member and structure yield displacement and may be approximated by Equation 4.6 [24].

$$
\Delta_{y}=\frac{\phi_{y} L^{2}}{3}
$$


The member and structure plastic displacement $\Delta_{p}$ is calculated by

$$
\Delta_{p}=\left(\Omega_{o}-1\right) \Delta_{y}+L_{p} \phi_{p}\left(L-0.5 L_{p}\right)
$$

where $L$ is the distance from the critical section of the plastic hinge to the point of contra-flexure and $L_{p}$ is the plastic hinge length. The plastic hinge length is defined as the zone in which severe damage to concrete sections occurs. Since most damage occurs at curvatures greater than the yield curvature, $L_{p}$ can be related to the yield moment and the ultimate moment, and therefore the Section Overstrength (Equation 4.8). This approach works well when the moments increase with curvature, however, it is questionable for members that experience post-peak strength degradation [4]. FRP confined concrete increases in strength with curvature up to failure and therefore lends itself well to this relationship.

$$
L_{p}=L\left(1-\frac{1}{\Omega_{o}}\right)
$$

In cases where members have post-peak strength degradation, as with typical steel confined bridge columns, other plastic hinge length relationships have traditionally been used. The length $L_{p}$ for a typical bridge-bent where the column's plastic hinge forms against a supporting member can be calculated using Equation $4.9 ; f_{y}$ represents the yield stress and $d_{b}$ represents the nominal bar diameter of the longitudinal reinforcement [6] [24].

$$
L_{p}=0.08 L+0.15 f_{y} d_{b} \geq 0.3 f_{y} d_{b} \text { (in., ksi) }
$$


The member and structure ductility $\mu_{\Delta}$ is calculated by

$$
\mu_{\Delta}=\frac{\Delta_{u}}{\Delta_{y}}
$$

which with substitution becomes

$$
\mu_{\Delta}=\Omega_{o}+3\left(\mu_{\phi}-1\right) \frac{L_{p}}{L}\left(1-0.5 \frac{L_{p}}{L}\right)
$$

It is important to note that in this example the cantilever column is assumed to have rigid end connections and therefore the member and structure displacement and ductility are equal. If the flexibility of the bridge's footing and superstructure are considered, the structural displacement will be greater than the member displacement and the structure ductility will be less than the member ductility due to increases in yield displacements caused by additional rotations at the column's end connections prior to yielding.

As demonstrated, Section Plastic Curvature, Section Curvature Ductility, and the Section Overstrength Factor are cross section properties directly related to member displacement, ductility, flexural strength, and flexural overstrength. Therefore, they are important to consider so that strength loss or collapse from the cyclic inelastic loading of a seismic event is avoided. This is the reason they are chosen as the cross section properties analyzed in the FRP versus steel confinement comparison, and the FRP confinement parameter study to follow.

Moment-Curvature diagrams are also used to analyze how the different confinement types and parameters affect cross section flexural strength. To do this MomentCurvature diagrams for the different cross sections with an applied Axial Load Ratio of 0.2 
are presented. The different moment capacities of the various cross sections are then compared to demonstrate how the confinement type or parameter influences the cross section flexural strength.

Axial Force-Moment Interaction diagrams are used in the parameter study to analyze the effect each parameter has on a cross section's Maximum Moment, $M_{m}$. The Maximum Moment is the largest moment a cross section can achieve, occurring at a unique axial load. $M_{m}$ is used to evaluate how each parameter influences the maximum possible overstrength of a cross section considering all feasible axial loads.

Additionally, the curvatures, moments, and axial loads determined in the MomentCurvature and PM Interaction diagrams are normalized and analyzed to determine the nondimensional effects of each parameter. Curvatures are normalized by multiplying them by the cross section diameter (in inches) as demonstrated in Equation 4.12. Equation 4.13 normalizes the moments by dividing them by the product of the unconfined concrete strength, the gross cross section area, and the section diameter (in feet). The normalized Axial Loads are calculated by Equation 4.14 which divides the Axial Load by the product of the unconfined concrete strength and the gross cross section area. Analyzing normalized moments and normalized axial loads allows the relative effects of a parameter and the efficiency of each cross section to be measured.

$$
\begin{gathered}
\phi_{N}=\phi D \\
M_{N}=\frac{M}{f_{c}^{\prime} A_{g} D}
\end{gathered}
$$




$$
P_{N}=\frac{P}{f_{c}^{\prime} A_{g}}
$$

\subsection{Confinement Comparison}

The FRP confined reinforced concrete circular cross section and a steel hoop confined reinforced concrete circular cross section comparison is conducted to establish FRP confinement as a possible supplement (or alternate) to steel confinement in seismic regions. To achieve this, Moment-Curvature analyses are used to determine if FRP materials can confine reinforced concrete as effectively as steel while identifying differences between the two confining systems that must be taken into account. The two cross sections chosen have the same diameter, longitudinal reinforcement, and unconfined concrete strength. The quantities of the two different cross sections' confinement are such that they will have similar Section Plastic Curvature Capacities, and therefore, similar displacement capacities at applied Axial Load Ratios between 0.1 and 0.5. Table 4.1 shows each of the two cross sections' properties used in the comparative study.

Figure 4.1 (a) shows the Moment-Curvature diagrams for both the FRP confined and steel confined cross sections with an applied Axial Load Ratio of 0.2. To compare the Moment-Curvature analyses of the two cross sections, their diagrams are broken up into three different zones of interest.

Zone 1, the initial portion of both the steel confined and FRP confined MomentCurvature diagrams, are nearly identical for curvatures between 0 and 0.00015 1/inch. At small curvatures the load combination on the cross section is not large enough to cause 
TABLE 4.1: Confinement Comparison Cross Section Configurations

\begin{tabular}{|l|l|l|}
\hline Section Property & Steel Section & FRP Section \\
\hline Section Diameter: & $24.00 \mathrm{in}$ & $24.00 \mathrm{in}$ \\
\hline Gross Section Area: & $452.39 \mathrm{sqin}$ & $452.39 \mathrm{sqin}$ \\
\hline Unconfined Concrete strength: & $4.00 \mathrm{ksi}$ & $4.00 \mathrm{ksi}$ \\
\hline Unconfined Concrete Elastic Modulus: & $57.00 \sqrt{1000 f_{c}^{\prime}} \mathrm{ksi}$ & $47.59 \sqrt{1000 f_{c}^{\prime}} \mathrm{ksi}$ \\
\hline Number of Longitudinal Bars: & 8 & 8 \\
\hline Longitudinal Bar Size: & $\# 7$ & $\# 7$ \\
\hline Longitudinal Bar Area: & $4.80 \mathrm{sqin}$ & $4.80 \mathrm{sqin}$ \\
\hline Longitudinal Reinforcing Ratio: & 0.011 & 0.011 \\
\hline Reinforcing Steel Yield Stress: & $60.00 \mathrm{ksi}$ & $60.00 \mathrm{ksi}$ \\
\hline Reinforcing Steel Fracture Stress: & $90.00 \mathrm{ksi}$ & $90.00 \mathrm{ksi}$ \\
\hline Reinforcing Steel Strain at Strain Hardening: & 0.0080 & 0.0080 \\
\hline Reinforcing Steel Failure Strain: & 0.090 & 0.090 \\
\hline Reinforcing Steel Elastic Modulus: & $29,000.00 \mathrm{ksi}$ & $29,000.00 \mathrm{ksi}$ \\
\hline Applied Axial Load: & $362.00 \mathrm{kips}$ & $362.00 \mathrm{kips}$ \\
\hline Transverse Reinforcement Material: & Steel & Carbon/Epoxy \\
\hline Type of Transverse Reinforcement: & Steel Hoop & Tyfo®SCH-41S \\
\hline Transverse Reinforcing Bar Size: & $\# 4$ & N/A \\
\hline Spacing of Transverse Reinforcement: & 4.00 in & N/A \\
\hline Cover Concrete Thickness: & 1.50 in & N/A \\
\hline Number of Cross Section Materials: & 3 & 2 \\
\hline Number of Composite Layers: & N/A & 6 \\
\hline Composite Layer Thickness: & N/A & 0.040 in \\
\hline Ultimate Fiber Fracture Stress: & N/A & $107.95 \mathrm{ksi}$ \\
\hline Effective Fiber Fracture Stress: & N/A & $53.98 \mathrm{ksi}$ \\
\hline Fiber Tensile Elastic Modulus: & N/A & $8,996.00 \mathrm{ksi}$ \\
\hline Ultimate Fiber Fracture Strain: & N/A & 0.012 \\
\hline Effective Fiber Fracture Strain: & N/A & 0.0060 \\
\hline
\end{tabular}




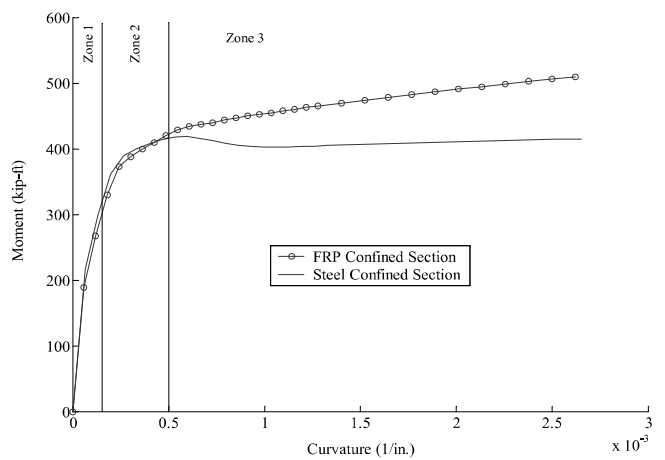

(a) moment-curvature

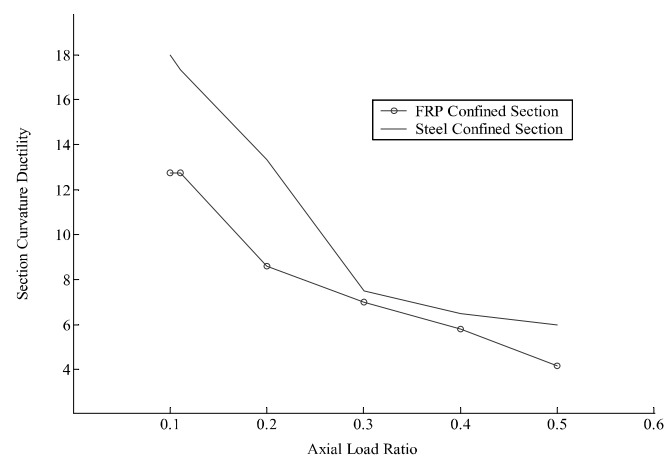

(c) section curvature ductility $\mathrm{w} /$ first material yielding limit state

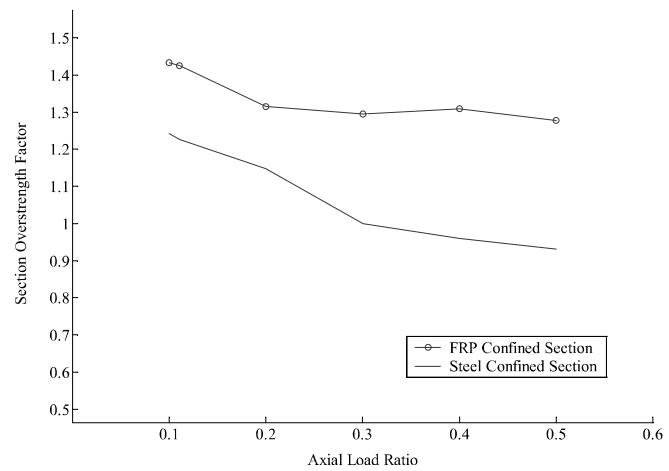

(e) section overstrength factor

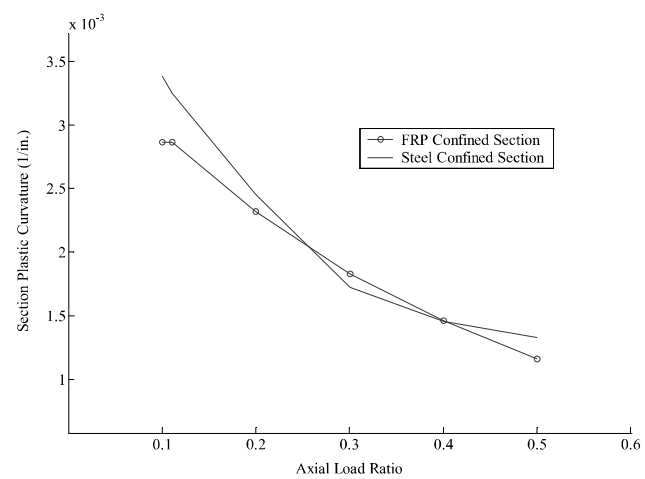

(b) section plastic curvature

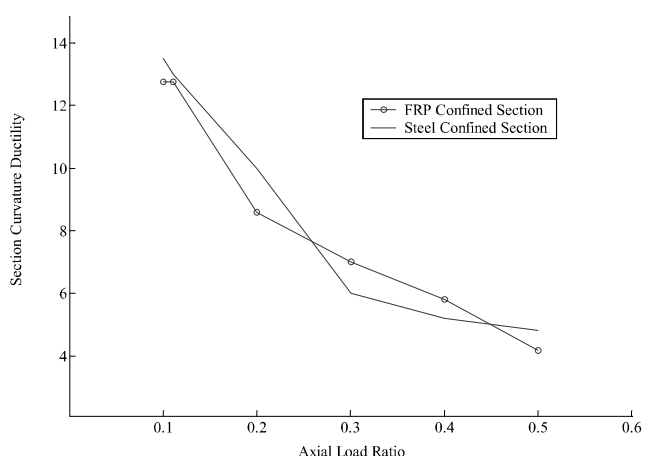

(d) section curvature ductility excluding cover concrete yielding limit state

FIGURE 4.1: Steel and FRP Confinement Comparison Figures 
significant concrete cracking. Therefore, the core concrete does not laterally dilate enough to fully engage the cross section's confining mechanism. Thus, at small curvatures the stiffness of the cross section is controlled by the initial stiffness of the concrete. Since the two cross sections are similar, except for their confinement which in this zone is not fully engaged, the diagrams are also similar. It should be noted, however, the FRP confinement model uses a slightly more conservative estimate of the initial concrete stiffness than the steel confinement model. This causes the FRP confined cross section's Moment-Curvature diagram to enter into its transition zone at a lower moment than the steel confined cross section.

Zone 2 is the portion of the Moment-Curvature diagrams where the two cross sections transition between the initial concrete stiffness and the confined concrete stiffness. This zone corresponds to curvatures between 0.00015 and $0.000501 /$ inch. Here, as the curvature increases, the core concrete increasingly cracks causing it to lose strength and stiffness and thus, laterally dilate. This dilation causes the confining mechanism to fully engage, preventing the core concrete from further losing strength and failing. At the end of this zone, the cross section stiffness and, thus, the shape of the Moment-Curvature diagrams is controlled by the confined concrete stiffness.

Zone 3 of the two Moment-Curvature diagrams, with curvatures of 0.00050 1/inch to failure, is where the steel confinement yields and strain hardens. The cover concrete of the steel confined cross section spalls off in this zone as well. It is in this section of the diagrams that the steel confined and FRP confined cross section properties diverge. The FRP confinement is linear elastic up to failure producing a constant and positive cross 
section stiffness. This causes a steady increase in the cross section's moment capacity up to failure. Conversely, the steel confinement transitions through yielding and then strain hardening, causing the cross section's stiffness to vary. When the steel confinement yields, it allows the cross section to dilate without requiring additional load. Thus, while the steel is yielding there is no increase in moment for an increase in curvature. Once the steel confinement begins to strain harden, the cross section's stiffness also begins to increase resulting in an increase in its moment capacity. It is also demonstrated in this zone that at curvatures between 0.00050 and 0.001001 /inch, the steel confined cross section has a decrease in stiffness. This is caused by spalling of the cross section's cover concrete. The FRP confined cross section, due to its external confinement application effectively confining the entire cross section, is not susceptible to cover concrete spalling and therefore does not exhibit the associated decrease in stiffness. The increase in moment capacity up to failure and the lack of cover concrete spalling are two properties where FRP confinement out performs steel confinement. However, because of this, FRP confined cross sections have a disadvantage over steel confined cross sections. Unlike steel confined cross sections, FRP confined sections display little to no warning signs that failure is approaching.

The Moment-Curvature diagram shown in Figure 4.1 (a) is one of a series of Moment-Curvature analyses conducted on the steel confined and FRP confined cross sections. The series of Moment-Curvature analyses is conducted to assess the Section Plastic Curvature, Section Curvature Ductility and Section Overstrength Factors of the two cross sections at Axial Load Ratios between 0.1 and 0.5. This will demonstrate how the two confinement systems influence the member displacement capacity, member ductility, flexural 
strength, and flexural overstrength.

The confinement comparison study shows that the rotational capacity of the two cross sections are very similar over the range of axial loads studied (Figure 4.1 (b)). The rotational capacities of the two cross sections are so similar that as the axial load increases they have alternating maximum Section Plastic Curvature values. Therefore, both the Steel confinement and the FRP confinement achieve the same member displacement capacities.

The steel confinement and FRP confinement comparison shows that the steel confined cross section has greater Section Curvature Ductility than the FRP confined section (Figure $4.1(\mathrm{c})$ ). Even though the two cross sections have similar Ultimate Curvatures, this occurs due to the steel confined cross section having a lower Yield Curvature. The low Yield Curvature is caused by the steel confined cross section's cover concrete yielding at a lower strain than the confined concrete and/or the longitudinal reinforcement of the FRP confined section. Therefore, though the steel confined cross section has greater Section Curvature Ductility it does not necessarily perform better under seismic excitation. To demonstrate this, an additional series of Moment-Curvature analyses is conducted using longitudinal reinforcement and confined core concrete yielding as the possible first yield limit states, disregarding the unconfined cover concrete yielding limit state. The MomentCurvature analyses using these limit states show both the steel confined and FRP confined cross sections have very similar Section Curvature Ductility values (Figure 4.1 (d)).

The comparison shows that the FRP confined section has a greater Section Overstrength Factor than the steel confined cross section over the entire range of Axial Load Ratios studied (Figure 4.1 (e)). Therefore, the FRP confined cross section has greater 
flexural strength and because both sections have similar Section Curvature Ductilities the FRP confinement produces greater member ductility. This is attributed to the linear elastic behavior of FRP confinement up to failure, yielding of the steel confinement prior to failure, and cover concrete spalling of the steel confined cross section. The Section Overstrength Factors for both cross sections decreases as the Axial Load Ratio increases; however, the FRP confined cross section decreases at a slower rate. In addition, it should be noted that at an Axial Load Ratio of 0.28 and greater, the steel confined cross section has a Section Overstrength Factor less than one. Section Overstrength Ratios less than one result in a drastic loss of member ductility.

This comparative study demonstrates that the steel confined and FRP confined cross sections both have initial stiffnesses that are primarily dependent on the concrete compressive strength. Then, as the curvatures imposed on the cross sections increase, the cross sections have similar stiffness transitions zones. However, the two cross sections have different secondary cross section stiffnesses due to the different properties of the steel and FRP confinement. The different confinement material properties give the FRP confined section advantages and disadvantages to the steel confined section. The FRP confined cross section has the advantage that its flexural strength and ductility increase up to failure and that the entire cross section is effectively confined where the steel confined cross section has a decrease in flexural strength and its cover concrete spalls. However, because of this FRP confined cross sections have a disadvantage to steel confined cross sections. Since there is no spalling of cover concrete and/or yield plateau, FRP confined sections provide little or no warning of approaching failure. 
This study also shows that, as expected, increasing the Axial Load Ratio cause both the steel confined and FRP confined cross sections to lose rotational capacity, ductility, and flexural strength. It is also demonstrated that FRP confinement can be as effective as steel confinement to achieve good member displacement capacity. It is shown that the Section Curvature Ductility of the steel confined section is greater than the FRP confined section; however, this is due to the steel confined section's cover concrete yielding early. Further analysis shows that both cross sections have very similar Section Curvature Ductility values when yielding of the unconfined cover concrete is not considered for the first material yielding limit state. It is shown that the FRP confined cross section has greater Section Overstrength Factors than the steel confined cross section. This means that the structure using FRP confinement has greater member ductility and flexural strength than the Structure using steel confinement. Thus, it can be concluded that FRP composites do have the ability to confine concrete as effectively, if not better than steel.

\subsection{Parameter Study}

The parameter studies are used to identify trends between design variables and member displacement capacity, ductility, flexural strength, and flexural overstrength. This is done to improve seismic design of FRP confined plastic hinges and surrounding capacity protected elastic components. The design variables studied include the 28 -day unconfined concrete strength $\left(f_{c}^{\prime}\right)$, longitudinal reinforcing ratio $\left(\frac{A_{s t}}{A_{g}}\right)$, cross section diameter $(D)$, confinement jacket thickness-cross section diameter ratio $\left(\frac{n t}{D}\right)$, and the FRP confinement system (System) of the cross section. 
The cross section configurations used in each study can be found in Table 4.2 and the rationale for choosing the parameter quantities follows. The $f_{c}^{\prime}$ parameter study analyzes unconfined concrete compressive strengths between 3.00 and $6.00 \mathrm{ksi}$. ACI 31805 Chapter 21 Section 21.2.4.1 limits $f_{c}^{\prime}$ to be no less than 3.00 ksi for seismic design [1]. Concrete with compressive strengths greater than $6.00 \mathrm{ksi}$ are considered high-strength and are beyond the scope of this study. Therefore, the $f_{c}^{\prime}$ parameter is broken up into seven cases starting with $3.00 \mathrm{ksi}$ and increasing in increments of $0.50 \mathrm{ksi}$ up to $6.00 \mathrm{ksi}$.

The $\frac{A_{s t}}{A_{g}}$ parameter, where $A_{s t}$ and $A_{g}$ are the areas of the longitudinal reinforcement and cross section, respectively, is required by ACI 318-05 Chapter 10 Section 10.9.1 to be between $1 \%$ and $8 \%$. However, for seimic design, ACI 318-05 Chapter 21 Section 21.4.3.1 reduces the upper limit to 6\% [1]. Additionally, it is recommended in Seismic Design and Retrofit of Bridges that for seismic design of circular columns, $\frac{A_{s t}}{A_{g}}$ should be between $0.5 \%$ and $4 \%$ with practical designs having limits of $1 \%$ and $3 \%$ [24]. The lower limit is set to control time-dependent deformations and to ensure the column's flexural strength is greater than its cracking strength [1] [24]. The upper longitudinal reinforcement limit is set to avoid reinforcement congestion, decrease sensitivity to $P-\Delta$ effects, reduce large amounts of transverse reinforcement required to restrain longintudinal reinforcement from buckling, and prevent large joint shear stresses [1] [24]. This study uses $\frac{A_{s t}}{A_{g}}$ limits of $0.5 \%$ and $6 \%$. These limits are used so the widest range of recommended values for seismic design are reviewed.

The $D$ parameter study analyzes seven cross sections varying in diameter from 12 to 60 inches. The diameters are chosen to be common sizes found throughout many 
structures.

The $\frac{n t}{D}$ parameter study involves seven different cross section configurations of various diameters and confinement quantities. Cross section diameters of 24 to 60 inches and FRP confinement layers in quantities of four to 18 are used to produce a range of $\frac{n t}{D}$ ratios from 0.0067 to 0.0167 . Four different cross section diameter sizes are used to determine if the effect of the $\frac{n t}{D}$ ratio parameter is consistent for various cross section sizes or if this parameter needs to be modified to account for size effects. Most FRP research focuses on data sets of cross sections having between one and 14 FRP confinement layers. However, it has been found that cross sections with only a few FRP layers fail in shear, and are therefore undesirable [32]. This study focuses on ductile behavior of cross sections, limiting the minimum number of FRP confinement layers to three.

The System parameter study involves five different FRP systems from two different manufacturers. The five FRP systems are selected to provide a wide range of FRP materials (Carbon, Glass, and Aramid), strengths, and ultimate strains. A list of the FRP systems used as well as their properties can be found in Table 4.3.

\subsubsection{Unconfined Concrete Compressive Strength Parameter Study}

The $f_{c}^{\prime}$ parameter study results (Table 4.4) show that increasing concrete compressive strength only slightly influences $\phi_{u}$ (Figure A.1 (a)) and $\phi_{p}$ (Figure A.1 (c)). Thus, the concrete compressive strength does not have a significant influence on cross section rotational capacity and therefore does not change the member displacement capacity. Though the displacement capacity is not significantly influenced, the member ductility is reduced by increasing $f_{c}^{\prime}$. The $f_{c}^{\prime}$ parameter only slightly influences $\mu_{\phi}$ (Figure A.1 (d)), which like 
TABLE 4.2: Parameter Study Cross Section Configurations

\begin{tabular}{|c|c|c|c|c|c|c|c|}
\hline Parameter & $\begin{array}{l}\text { f'c } \\
(\mathrm{ksi})\end{array}$ & $\begin{array}{l}\text { Long. } \\
\text { Rein. }\end{array}$ & $\begin{array}{l}A_{s t} / A_{g} \\
(\%)\end{array}$ & $\begin{array}{l}\text { D } \\
\text { (in.) }\end{array}$ & $\begin{array}{l}\text { n (FRP } \\
\text { Layers) }\end{array}$ & nt/D & $\begin{array}{l}\text { System } \\
\text { (FRP } \\
\text { Material) }\end{array}$ \\
\hline $\mathrm{f}^{\prime} \mathrm{c}$ & 3.00 & $\overline{~(8) \# 7}$ & 1.06 & 24.00 & $\overline{4}$ & 0.0067 & Carbon 108 \\
\hline$f^{\prime} c$ & 3.50 & $(8) \# 7$ & 1.06 & 24.00 & 4 & 0.0067 & Carbon 108 \\
\hline$f^{\prime \prime c}$ & 4.00 & $(8) \# 7$ & 1.06 & 24.00 & 4 & 0.0067 & Carbon 108 \\
\hline f'c & 4.50 & $(8) \# 7$ & 1.06 & 24.00 & 4 & 0.0067 & Carbon 108 \\
\hline f'c & 5.00 & $(8) \# 7$ & 1.06 & 24.00 & 4 & 0.0067 & Carbon 108 \\
\hline$f^{\prime} c$ & 5.50 & $(8) \# 7$ & 1.06 & 24.00 & 4 & 0.0067 & Carbon 108 \\
\hline f'c & 6.00 & $(8) \# 7$ & 1.06 & 24.00 & 4 & 0.0067 & Carbon 108 \\
\hline 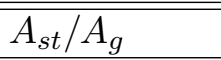 & "4.00 & (8)\#7 & 1.06 & 24.00 & 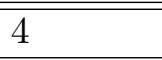 & 0.0067 & Carbon 108 \\
\hline$A_{s t} / A_{g}$ & 4.00 & $(9 \# 9)$ & 1.99 & 24.00 & 4 & 0.0067 & Carbon 108 \\
\hline$A_{s t} / A_{g}$ & 4.00 & $(11) \# 10$ & 3.09 & 24.00 & 4 & 0.0067 & Carbon 108 \\
\hline$A_{s t} / A_{g}$ & 4.00 & $(14) \# 10$ & 3.93 & 24.00 & 4 & 0.0067 & Carbon 108 \\
\hline$A_{s t} / A_{g}$ & 4.00 & $(10) \# 14$ & 4.97 & 24.00 & 4 & 0.0067 & Carbon 108 \\
\hline$A_{s t} / A_{g}$ & 4.00 & $(12) \# 14$ & 5.97 & 24.00 & 4 & 0.0067 & Carbon 108 \\
\hline$A_{s t} / A_{g}$ & 4.00 & $(14) \# 14$ & 6.96 & 24.00 & 4 & 0.0067 & Carbon 108 \\
\hline$A_{s t} / A_{g}$ & 4.00 & (9)\#18 & 7.96 & 24.00 & 4 & 0.0067 & Carbon 108 \\
\hline$\overline{\mathrm{D}}$ & 4.00 & $\overline{(26) \# 4}$ & 1.06 & 12.00 & 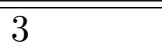 & 0.0100 & Carbon 108 \\
\hline $\mathrm{D}$ & 4.00 & $(6) \# 6$ & 1.04 & 18.00 & 5 & 0.0111 & Carbon 108 \\
\hline $\mathrm{D}$ & 4.00 & $(8) \# 7$ & 1.06 & 24.00 & 6 & 0.0100 & Carbon 108 \\
\hline $\mathrm{D}$ & 4.00 & $(12) \# 7$ & 1.02 & 30.00 & 8 & 0.0107 & Carbon 108 \\
\hline $\mathrm{D}$ & 4.00 & $(10) \# 9$ & 0.98 & 36.00 & 9 & 0.0100 & Carbon 108 \\
\hline $\mathrm{D}$ & 4.00 & $(14) \# 10$ & 0.98 & 48.00 & 12 & 0.0100 & Carbon 108 \\
\hline $\mathrm{D}$ & 4.00 & $(14) \# 14$ & 1.11 & 60.00 & 15 & 0.0100 & Carbon 108 \\
\hline nt/D & 4.00 & (8)\#7 & 21.06 & 24.00 & 4 & 0.0067 & Carbon 108 \\
\hline $\mathrm{nt} / \mathrm{D}$ & 4.00 & $(14) \# 10$ & 0.98 & 48.00 & 10 & 0.0083 & Carbon 108 \\
\hline $\mathrm{nt} / \mathrm{D}$ & 4.00 & $(8) \# 7$ & 1.06 & 24.00 & 6 & 0.0100 & Carbon 108 \\
\hline $\mathrm{nt} / \mathrm{D}$ & 4.00 & $(14) \# 14$ & 1.11 & 60.00 & 18 & 0.0120 & Carbon 108 \\
\hline $\mathrm{nt} / \mathrm{D}$ & 4.00 & $(8) \# 7$ & 1.06 & 24.00 & 8 & 0.0133 & Carbon 108 \\
\hline $\mathrm{nt} / \mathrm{D}$ & 4.00 & $(10) \# 9$ & 0.98 & 36.00 & 14 & 0.0156 & Carbon 108 \\
\hline $\mathrm{nt} / \mathrm{D}$ & 4.00 & $(8) \# 7$ & 1.06 & 24.00 & 10 & 0.0167 & Carbon 108 \\
\hline System & 4.00 & $(8) \# 7$ & 1.06 & 24.00 & 6 & 0.0100 & Carbon 108 \\
\hline System & 4.00 & $(8) \# 7$ & 1.06 & 24.00 & 5 & 0.0104 & Aramid \\
\hline System & 4.00 & $(8) \# 7$ & 1.06 & 24.00 & 5 & 0.0104 & S-Glass \\
\hline System & 4.00 & $(8) \# 7$ & 1.06 & 24.00 & 6 & 0.0100 & E-Glass \\
\hline System & 4.00 & $(8) \# 7$ & 1.06 & 24.00 & 6 & 0.0100 & Carbon 97 \\
\hline
\end{tabular}


TABLE 4.3: FRP Confinement System Properties

\begin{tabular}{|c|c|c|c|c|}
\hline $\begin{array}{l}\text { System } \\
\text { (FRP } \\
\text { Material) }\end{array}$ & $\begin{array}{l}\text { FRP } \\
\text { Product } \\
\text { Name } \\
\end{array}$ & $\begin{array}{l}\text { FRP Layer } \\
\text { Thickness - } \\
\text { t (in.) }\end{array}$ & $\begin{array}{l}\text { Ult. Fiber } \\
\text { Stress - } \\
f_{f u}(\mathrm{ksi})\end{array}$ & $\begin{array}{l}\text { Ult. Fiber } \\
\text { Strain - } \\
e_{f u} \text { (in./in.) }\end{array}$ \\
\hline Carbon 108 & TyfoßSCH-41S & 0.040 & 107.95 & 0.012 \\
\hline Aramid & Tyfo®SCH-41 & 0.050 & 80.80 & 0.017 \\
\hline S-Glass & TyfoßSEH-51A & 0.050 & 66.70 & 0.022 \\
\hline E-Glass & SikaWrap@1006 & 0.040 & 74.60 & 0.020 \\
\hline Carbon 97 & SikaWrap@103C & 0.040 & 97.00 & 0.010 \\
\hline
\end{tabular}

TABLE 4.4: Effects of Increasing the $f^{\prime} c$ Parameter

\begin{tabular}{|c|c|c|c|}
\hline $\begin{array}{l}\text { Section } \\
\text { Property }\end{array}$ & Effect & Influence & Special Notes \\
\hline$\phi_{y}$ & $\begin{array}{l}\text { Increases } \\
\text { Linearly }\end{array}$ & Very Small & $\begin{array}{l}\text { Influence increases with axial load, values } \\
\text { diverge }\end{array}$ \\
\hline $\begin{array}{l}\text { Normalized } \\
\phi_{y}\end{array}$ & $\begin{array}{l}\text { Increases } \\
\text { Linearly }\end{array}$ & Very Small & $\begin{array}{l}\text { Influence increases with axial load, values } \\
\text { diverge }\end{array}$ \\
\hline$M_{y}$ & $\begin{array}{l}\text { Increases } \\
\text { Linearly }\end{array}$ & Large & $\begin{array}{l}\text { Influence increases with axial load, values } \\
\text { diverge }\end{array}$ \\
\hline $\begin{array}{l}\text { Normalized } \\
M_{y}\end{array}$ & $\begin{array}{l}\text { Decreases } \\
\text { Nonlinearly }\end{array}$ & Large & $\begin{array}{l}\text { Influence increases with axial load, values } \\
\text { diverge }\end{array}$ \\
\hline$\phi_{u}$ & Varies & Very Small & $\begin{array}{l}\text { Increases for } P_{r}<0.2 \text { and Decreases for } \\
P_{r} \geq 0.2\end{array}$ \\
\hline $\begin{array}{l}\text { Normalized } \\
\phi_{u}\end{array}$ & Varies & Very Small & $\begin{array}{l}\text { Increases Linearly for } P_{r}<0.2 \text { and De- } \\
\text { creases Linearly for } P_{r} \geq 0.2\end{array}$ \\
\hline$M_{u}$ & $\begin{array}{l}\text { Increases } \\
\text { Linearly }\end{array}$ & Large & $\begin{array}{l}\text { Influence increases with axial load, values } \\
\text { diverge }\end{array}$ \\
\hline $\begin{array}{l}\text { Normalized } \\
M_{u}\end{array}$ & $\begin{array}{l}\text { Decreases } \\
\text { Nonlinearly }\end{array}$ & Large & $\begin{array}{l}\text { Influence increases with axial load, values } \\
\text { diverge }\end{array}$ \\
\hline$\phi_{p}$ & Varies & Very Small & $\begin{array}{l}\text { Increases for } P_{r}<0.2 \text { and Decreases for } \\
P_{r} \geq 0.2\end{array}$ \\
\hline$\mu_{\phi}$ & Varies & Very Small & $\begin{array}{l}\text { Increases for } P_{r}<0.2 \text { and Decreases for } \\
P_{r} \geq 0.2\end{array}$ \\
\hline$\Omega_{o}$ & Decreases & Small & \\
\hline$M_{m}$ & $\begin{array}{l}\text { Increases } \\
\text { Linearly }\end{array}$ & Large & \\
\hline $\begin{array}{l}\text { Normalized } \\
M_{m}\end{array}$ & $\begin{array}{l}\text { Decreases } \\
\text { Nonlinearly }\end{array}$ & Large & \\
\hline
\end{tabular}


$\phi_{p}$, increases with Axial Load Ratios less than 0.2, and decreases with Axial Load Ratios greater than 0.2. However, $\Omega_{o}$ (Figure A.1 (e))has small but significant decreases as the concrete strength increases. Thus, increasing $f_{c}^{\prime}$ decreases the member ductility.

Increasing $f_{c}^{\prime}$ linearly increases cross section moment capacity and therefore increases the member flexural strength and maximum feasible member flexural overstrength (Figures A.1 (a), (e), and (f)). Though the flexural strength increases, the normalized flexural strength decreases (Figure A.1 (b)) meaning the cross section is less efficient with high concrete compressive strengths. This occurs because only the compressive strength of concrete increases, not the confinement of the cross section. Since there is no increase in confinement, the added strength of the cross section is attributed solely to the increase in $f_{c}^{\prime}$. This agrees well with the relationship founded by Considére [10] and shown in Equation 2.1 which demonstrates that the enhanced strength of confined concrete is a function of both the unconfined concrete strength and the available confinement pressure. In this case, the available confinement pressure remains constant. Thus, the enhanced confined concrete strength is solely a function of the increasing unconfined concrete strength. To increase the normalized flexural strength and therefore the efficiency of the cross sections, additional confinement should be added with increasing unconfined concrete strengths.

\subsubsection{Longitudinal Reinforcing Ratio Parameter Study}

The results from the $\frac{A_{s t}}{A_{g}}$ parameter study (Table 4.5) show that increasing the amount of longitudinal reinforcing steel decreases the rotational capacity of a cross section and therefore decreases the displacement capacity of a member. This occurs because as a cross section becomes over-reinforced the depth of the neutral axial increases causing first 
TABLE 4.5: Effects of Increasing the $\frac{A_{s t}}{A_{g}}$ Parameter

\begin{tabular}{|c|c|c|c|}
\hline $\begin{array}{l}\text { Section } \\
\text { Property }\end{array}$ & Effect & Influence & Special Notes \\
\hline$\phi_{y}$ & $\begin{array}{l}\text { Decreases } \\
\text { Nonlinearly }\end{array}$ & Very Small & \\
\hline $\begin{array}{l}\text { Normalized } \\
\phi_{y}\end{array}$ & $\begin{array}{l}\text { Decreases } \\
\text { Nonlinearly }\end{array}$ & Very Small & \\
\hline$M_{y}$ & $\begin{array}{l}\text { Increases } \\
\text { Linearly }\end{array}$ & Large & \\
\hline $\begin{array}{l}\text { Normalized } \\
M_{y}\end{array}$ & $\begin{array}{l}\text { Increases } \\
\text { Linearly }\end{array}$ & Large & \\
\hline$\phi_{u}$ & $\begin{array}{l}\text { Decreases } \\
\text { Nonlinearly }\end{array}$ & Large & $\begin{array}{l}\text { Influence decreases as axial load increases, } \\
\text { values converge }\end{array}$ \\
\hline $\begin{array}{l}\text { Normalized } \\
\phi_{u}\end{array}$ & $\begin{array}{l}\text { Decreases } \\
\text { Nonlinearly }\end{array}$ & Large & $\begin{array}{l}\text { Influence decreases as axial load increases, } \\
\text { values converge }\end{array}$ \\
\hline$M_{u}$ & $\begin{array}{l}\text { Increases } \\
\text { Linearly }\end{array}$ & Large & \\
\hline $\begin{array}{l}\text { Normalized } \\
M_{u}\end{array}$ & $\begin{array}{l}\text { Increases } \\
\text { Linearly }\end{array}$ & Large & \\
\hline$\phi_{p}$ & $\begin{array}{l}\text { Decreases } \\
\text { Nonlinearly }\end{array}$ & Large & $\begin{array}{l}\text { Influence decreases as axial load increases, } \\
\text { values converge }\end{array}$ \\
\hline$\mu_{\phi}$ & $\begin{array}{l}\text { Decreases } \\
\text { Nonlinearly }\end{array}$ & Large & $\begin{array}{l}\text { Influence decreases as axial load increases, } \\
\text { values converge }\end{array}$ \\
\hline$\Omega_{o}$ & $\begin{array}{l}\text { Increases } \\
\text { Nonlinearly }\end{array}$ & Very Small & $\begin{array}{l}\text { Cross Section w/ } \frac{A_{s t}}{A_{g}}=0.6 \% \text { (smallest) } \\
\text { has much lower value than the rest }\end{array}$ \\
\hline$M_{m}$ & $\begin{array}{l}\text { Increases } \\
\text { Linearly }\end{array}$ & Large & \\
\hline $\begin{array}{l}\text { Normalized } \\
M_{m}\end{array}$ & $\begin{array}{l}\text { Increases } \\
\text { Linearly }\end{array}$ & Large & \\
\hline
\end{tabular}


failure of the extreme concrete compression fiber to occur at smaller curvatures. Thus, $\phi_{u}$ and $\phi_{p}$ are reduced and in turn the member displacement capacity is also reduced (Figures A.2 (a) and (c)).

Similarly, member ductility is also decreased. Increasing $\frac{A_{s t}}{A_{g}}$ decreases $\mu_{\phi}$ (Figure A.2 (d)) which decreases member ductility. Also, other than at very low amounts of longitudinal reinforcement $(0.5 \%)$ increasing $\frac{A_{s t}}{A_{g}}$ does not significantly increase $\Omega_{o}$ (Figure A.2 (e)). Thus, their accumulative effect is decreasing member ductility.

Additionally, as expected, increasing axial loads decrease the member displacement capacity and ductility; however, increasing axial loads also reduces the influence of the $\frac{A_{s t}}{A_{g}}$ parameter on theses properties. Increasing the axial load on a cross section increases the depth of the neutral axis which increases the concrete compression zone area while decreasing the amount of tensile strain and therefore stress in the longitudinal reinforcement. This reduces the influence of the longitudinal reinforcement, resulting in converging rotational capacities and ductility.

Increasing $\frac{A_{s t}}{A_{g}}$ increases both $M_{u}$ and $M_{m}$ as well as their normalized values (Figures A.2 (a), (b), and (f)). Therefore, increasing the amount of longitudinal reinforcement increases member flexural strength, the maximum feasible member overstrength, and the member efficiency. Thus, in seismic design, the amount of longitudinal reinforcement used should be selected to balance member flexural strength and efficiency with the necessary member displacement capacity and ductility. If displacement and ductility control design, a cross section with an Axial Load Ratio of 0.2 or less and a low $\frac{A_{s t}}{A_{g}}$ between $1 \%$ and $3 \%$ should be used. If flexural strength and efficiency control design, Axial Load Ratios between 
TABLE 4.6: Effects of Increasing the $D$ Parameter

\begin{tabular}{|c|c|c|c|}
\hline $\begin{array}{l}\text { Section } \\
\text { Property }\end{array}$ & Effect & Influence & Special Notes \\
\hline$\phi_{y}$ & $\begin{array}{l}\text { Decreases } \\
\text { Nonlinearly }\end{array}$ & Large & $\begin{array}{l}\text { Influence increases with axial load, values } \\
\text { diverge }\end{array}$ \\
\hline $\begin{array}{l}\text { Normalized } \\
\phi_{y}\end{array}$ & $\begin{array}{l}\text { Decreases } \\
\text { Nonlinearly }\end{array}$ & Large & $\begin{array}{l}\text { Influence increases with axial load, values } \\
\text { diverge }\end{array}$ \\
\hline$M_{y}$ & $\begin{array}{l}\text { Increases } \\
\text { Nonlinearly }\end{array}$ & Very Large & $\begin{array}{l}\text { Influence increases with axial load, values } \\
\text { diverge }\end{array}$ \\
\hline $\begin{array}{l}\text { Normalized } \\
M_{y}\end{array}$ & Converges & Very Small & \\
\hline$\phi_{u}$ & $\begin{array}{l}\text { Decreases } \\
\text { Nonlinearly }\end{array}$ & Very Large & $\begin{array}{l}\text { Influence decreases as axial load increases, } \\
\text { values converge }\end{array}$ \\
\hline $\begin{array}{l}\text { Normalized } \\
\phi_{u}\end{array}$ & Converges & Very Small & \\
\hline$M_{u}$ & $\begin{array}{l}\text { Increases } \\
\text { Nonlinearly }\end{array}$ & Very Large & $\begin{array}{l}\text { Influence increases with axial load, values } \\
\text { diverge }\end{array}$ \\
\hline $\begin{array}{l}\text { Normalized } \\
M_{u}\end{array}$ & Converges & Very Small & \\
\hline$\phi_{p}$ & $\begin{array}{l}\text { Decreases } \\
\text { Nonlinearly }\end{array}$ & Large & $\begin{array}{l}\text { Influence decreases as axial load increases, } \\
\text { values converge }\end{array}$ \\
\hline$\mu_{\phi}$ & $\begin{array}{l}\text { Increases } \\
\text { Nonlinearly }\end{array}$ & Small & $\begin{array}{l}\text { Values converge for column diameters } \\
\text { greater than } 24 \text { inches }\end{array}$ \\
\hline$\overline{\Omega_{o}}$ & $\begin{array}{l}\text { Increases } \\
\text { Nonlinearly }\end{array}$ & Small & $\begin{array}{l}\text { Values converge for column diameters } \\
\text { greater than } 24 \text { inches }\end{array}$ \\
\hline$M_{m}$ & $\begin{array}{l}\text { Increases } \\
\text { Nonlinearly }\end{array}$ & Very Large & \\
\hline $\begin{array}{l}\text { Normalized } \\
M_{m}\end{array}$ & Converges & Very Small & \\
\hline
\end{tabular}

0.2 and 0.4 with a $\frac{A_{s t}}{A_{g}}$ between $4 \%$ and $6 \%$ should be used.

\subsubsection{Varying Cross Section Diameter Parameter Study}

The $D$ parameter study results (Table 4.6) show that increasing cross section diameter greatly decreases $\phi_{u}$ and $\phi_{p}$ (Figures A.3 (a) and (c)). Thus, the cross section rotational capacity and, in turn, the member displacement capacity are also greatly reduced. Increasing the diameter increases the distance between the neutral axis and critical material 
fibers, increasing their strains at lower curvatures and thus causing earlier first material failure. This means that for seismic resistance the diameter of FRP confined cross sections should be controlled by the inelastic displacement requirements of the member and/or structure with flexural strength being important but of secondary concern.

Dissimilar to member displacement, the diameter only has a significant impact on member ductility for small cross sections. $\mu_{\phi}$ and $\Omega_{o}$ show significant increases as diameters increase up to 24 inches (Figures A.3 (d) and (e)). However, cross sections with diameters greater than 24 inches show no significant increase in $\mu_{\phi}$ or $\Omega_{o}$, and therefore, member ductility is not significantly increased.

As expected, it is shown that the cross section diameter has a very large influence on flexural strength and the maximum feasible flexural overstrength (Figure A.3 (a)). Increasing the diameter greatly increases the cross section compression zone area as well as the cross section moment-arm length. These two factors compound to greatly increase member flexural strength and maximum feasible member overstrength.

Though increasing the diameter greatly increases flexure strength, it does not necessarily increase efficiency. This parameter study shows that there is only a slight increase in the Normalized $M_{u}$ and the Normalized $M_{m}$ (Figures A.3 (b) and (f)). This demonstrates that while the diameter of a cross section has a large influence on flexural strength it does not have a significant influence on its efficiency. 
TABLE 4.7: Effects of Increasing the $\frac{n t}{D}$ Parameter

\begin{tabular}{|c|c|c|c|}
\hline $\begin{array}{l}\text { Section } \\
\text { Property }\end{array}$ & Effect & Influence & Special Notes \\
\hline$\phi_{y}$ & Decreases & Small & \\
\hline $\begin{array}{l}\text { Normalized } \\
\phi_{y}\end{array}$ & Decreases & Very Small & \\
\hline$M_{y}$ & Increases & Very Large & $\begin{array}{l}\text { Section diameter has very large influence, } \\
\text { small increases found with constant diam- } \\
\text { eter }\end{array}$ \\
\hline $\begin{array}{l}\text { Normalized } \\
M_{y}\end{array}$ & Increases & Very Small & \\
\hline$\phi_{u}$ & Decreases & Very Large & $\begin{array}{l}\text { Section diameter has very large influence, } \\
\text { large increases found with constant diam- } \\
\text { eter }\end{array}$ \\
\hline $\begin{array}{l}\text { Normalized } \\
\phi_{u}\end{array}$ & $\begin{array}{l}\text { Increases } \\
\text { Nonlinearly }\end{array}$ & Small & \\
\hline$M_{u}$ & $\begin{array}{l}\text { Increases } \\
\text { Nonlinearly }\end{array}$ & Very Large & $\begin{array}{l}\text { Section diameter has very large influence, } \\
\text { small increases found with constant diam- } \\
\text { eter }\end{array}$ \\
\hline $\begin{array}{l}\text { Normalized } \\
M_{u}\end{array}$ & $\begin{array}{l}\text { Increases } \\
\text { Nonlinearly }\end{array}$ & Small & \\
\hline$\phi_{p}$ & $\begin{array}{l}\text { Decreases } \\
\text { Nonlinearly }\end{array}$ & Large & $\begin{array}{l}\text { Section diameter has very large influence, } \\
\text { large nonlinear increases found with con- } \\
\text { stant diameter }\end{array}$ \\
\hline$\mu_{\phi}$ & $\begin{array}{l}\text { Increases } \\
\text { Nonlinearly }\end{array}$ & Small & \\
\hline$\Omega_{o}$ & $\begin{array}{l}\text { Increases } \\
\text { Nonlinearly }\end{array}$ & Small & \\
\hline$M_{m}$ & $\begin{array}{l}\text { Increases } \\
\text { Nonlinearly }\end{array}$ & Very Large & $\begin{array}{l}\text { Section diameter has very large influence, } \\
\text { small nonlinear increases found with con- } \\
\text { stant diameter }\end{array}$ \\
\hline $\begin{array}{l}\text { Normalized } \\
M_{m}\end{array}$ & $\begin{array}{l}\text { Increases } \\
\text { Nonlinearly }\end{array}$ & Small & \\
\hline
\end{tabular}




\subsubsection{FRP confinement Jacket Thickness-Cross Section Diameter Ratio Parameter Study}

The $\frac{n t}{D}$ parameter study shows (Table 4.7) that increasing the $\frac{n t}{D}$ ratio increases rotational capacity of the cross section, thus, increasing the member displacement capacity. This relationship is not linear, since as the $\frac{n t}{D}$ ratio increases it has less influence on $\phi_{u}$ and $\phi_{p}$ (Figures A.4 (a) and (c)). In addition, it is shown that the $\frac{n t}{D}$ ratio has less influence on rotational capacity as cross section diameter increases. Therefore, the cross section diameter has greater influence on the member displacement capacity than the $\frac{n t}{D}$ ratio. Thus, the cross section diameter is of primary concern for inelastic displacement capacity. The $\frac{n t}{D}$ ratio may therefore be controlled by ductility and/or flexural strength requirements.

As expected, increasing the $\frac{n t}{D}$ ratio increases $\mu_{\phi}$ and $\Omega_{o}$ (Figures A.4 (d) and (e)) and therefore, it also increases member ductility. Additionally, it is shown that as the $\frac{n t}{D}$ increases it has less influence on the member ductility, therefore having a nonlinear relationship. Thus, there is a limit to the $\frac{n t}{D}$ ratio after which any additional confinement jacket thickness will not increase ductility.

The $M_{u}$ (Figure A.4 (a)) and therefore the flexural strength is increased by increasing the $\frac{n t}{D}$ ratio. Like displacement capacity and ductility, this relationship is nonlinear, influence decreases at larger $\frac{n t}{D}$ ratios. Similarly, the maximum feasible flexural overstrength is also nonlinearly increased with the $\frac{n t}{D}$ ratio. It is also shown that both the Normalized $M_{u}$ and the Normalized $M_{m}$ values increase with the $\frac{n t}{D}$ ratio (Figures A.4 (b) and (f)). Therefore, increasing the $\frac{n t}{D}$ ratio also increases cross section efficiency. 
TABLE 4.8: FRP System Property $\left(f_{f u}\right.$ or $\left.e_{f u}\right)$ with Greatest Influence on Cross Section Properties

\begin{tabular}{|l|l|l|l|}
\hline $\begin{array}{l}\text { Section } \\
\text { Property }\end{array}$ & $\begin{array}{l}\text { FRP } \\
\text { Property }\end{array}$ & Influence & Special Notes \\
\hline$\phi_{y}$ & $e_{f u}$ & Very Small & \\
\hline $\begin{array}{l}\text { Normalized } \\
\phi_{y}\end{array}$ & $e_{f u}$ & Very Small & \\
\hline$M_{y}$ & $f_{f u}$ & Very Small & \\
\hline $\begin{array}{l}\text { Normalized } \\
M_{y}\end{array}$ & $f_{f u}$ & Very Small & \\
\hline$\phi_{u}$ & $e_{f u}$ & Large & \\
\hline $\begin{array}{l}\text { Normalized } \\
\phi_{u}\end{array}$ & $e_{f u}$ & Large & \\
\hline $\begin{array}{l}M_{u} \\
\text { Normalized } \\
M_{u}\end{array}$ & $f_{f u}$ & Small & $\begin{array}{l}\text { Carbon } 97 \text { does not fit this trend due to } \\
\text { its low } e_{f u}\end{array}$ \\
\hline$\phi_{p}$ & $e_{f u}$ & Small & $\begin{array}{l}\text { Carbon } 97 \text { does not fit this trend due to } \\
\text { its low } e_{f u}\end{array}$ \\
\hline$\mu_{\phi}$ & $e_{f u}$ & Large & \\
\hline$\Omega_{o}$ & $f_{f u}$ & Small & $\begin{array}{l}\text { Carbon } 97 \text { does not fit this trend due to } \\
\text { its low } e_{f u}\end{array}$ \\
\hline$M_{m}$ & $f_{f u}$ & Large & \\
\hline $\begin{array}{l}\text { Normalized } \\
M_{m}\end{array}$ & $f_{f y}$ & Large & \\
\hline
\end{tabular}

\subsubsection{Varying FRP System Parameter Study}

It is shown in the System parameter study (Table 4.8) that the cross sections confined by FRP systems with large $e_{f u}$ strains (S-Glass, E-Glass, and Aramid) have the largest $\phi_{u}$ and $\phi_{p}$ and therefore the largest member displacement capacities (Figures A.5 (a) and (c)). The S-Glass, E-Glass, and Aramid confined cross sections have the largest displacement capacities, even though the two cross section confined by carbon have much greater $f_{f u}$ stresses. Therefore, the most critical FRP system property for member displacement is its $e_{f u}$.

The cross sections confined by the Glass and Aramid FRP materials with greater 
rupture strains have the largest $\mu_{\phi}$ values (Figure A.4 (d)). Unlike $\mu_{\phi}, \Omega_{o}$ (Figure A.5 (e)) is most heavily influenced by the System strength, $f_{f u}$. Therefore the cross section confined by Carbon 108 with high tensile strength has the largest $\Omega_{o}$. However, the cross section confined by Carbon 97 has the lowest $\Omega_{o}$ even though it is stronger then the Glass and Aramid confinement systems. This is because Carbon 97 has the smallest rupture strain and unlike Carbon 108, it does not have enough tensile strength to resist large moments. Thus, to maximize member ductility for seismic design FRP cross section confinement systems should be selected to balance high strength and flexibility.

It is shown that at seismic design axial load levels, the flexural strength is influenced by a combination of FRP $f_{f u}$ and $e_{f u}$. However, as axial load increases, the influence of the FRP system $f_{f u}$ becomes far more important than the $e_{f u}$ (Figure A.5 (f)). Thus, $f_{f u}$ is the most critical FRP system property when considering the maximum feasible member flexural overstrength. This is an important factor to consider during the seismic design of frame column members where axial loads can significantly vary during seismic activity. 


\section{CHAPTER 5}

\section{Conclusion}

This study investigates the use of FRP confinement for reinforced concrete circular cross sections as a supplement or an alternate to typical steel confinement. The purpose is to increase the seismic performance of existing structures and ensure adequate seismic performance of new structures through the context of seismic design recommendations for FRP confined reinforced concrete. To achieve this, FRP confinement is compared to traditional steel confinement, determining whether FRP materials can confine concrete as effectively as steel. Additionally, the effect typical design variables have on member displacement capacity, ductility, flexural strength, and flexural overstrength are analyzed.

Based on the comparative study findings, it can be concluded that FRP composites can confine reinforced concrete circular cross sections as effectively as steel. Moreover, FRP confined cross sections can even display greater flexural strength and ductility than steel confined cross sections at equal inelastic displacements. Based on results, FRP materials can be a good supplement or even alternative to steel confinement for improving 
seismic performance of existing structures and ensuring adequate seismic performance of new structures.

A parameter study demonstrates that the cross section diameter is of primary concern to ensure inelastic displacement requirements are met. Next, the section ductility and flexural strength can be determined and adjusted as required. Results indicate that in order to maximize ductility and flexural strength, FRP confined circular cross sections should have the following characteristics: low to moderate $f_{c}^{\prime}$ between 3.00 and $4.50 \mathrm{ksi}$, low to moderate $\frac{A_{s t}}{A_{g}}$ between $1 \%$ and $3 \%$, cross section diameter not less than 24 inches, large $\frac{n t}{D}$ ratio, small Axial Load Ratio between 0.1 and 0.3 , and be confined by an FRP material with a large rupture strain. Plastic hinges rehabilitated or designed with these properties will maintain strength and have minimal damage during inealstic deformation cycles induced by code intensity seismic events, thus, increasing the seismic performance of both retrofitted existing and new structures. As compared with steel and properly adjusted within the most critical parameter limits, FRP confined circular cross sections meet and often surpass seismic design criteria of our most current codes. This alternate material as a supplement or on its own provides necessary performance to serve as a feasible and maybe even recommended option for structural applications. 


\section{BIBLIOGRAPHY}

[1] ACI Committee 318. ACI 318-05: Building Code Requirements for Structural Concrete and Commentary. American Concrete Institute, 2005.

[2] ACI Committee 440. ACI 440.2R-08: Guide for the Design and Construction of Externally Bonded FRP Systems for Strengthening Concrete Structures. American Concrete Institute, 2008.

[3] S. H. Ahmad and S. P. Shah. Stress-strain curves of concrete confined by spiral reinforcement. ACI Journal, 79(6):484 - 490, 1982.

[4] S. Bae and O Bayrak. Plastic hinge length of reinforced concrete columns. Technical report, ACI STRUCTURAL JOURNAL, May 2008.

[5] G. G. Balmer. Shearing strength of concrete under high triaxial stress - computation of mohr's envelope as a curve. Structural Research Laboratory Report SP-23, U. S. Bureau of Reclamation, Denver, 1949.

[6] Caltrans. Caltrans Seismic Design Criteria Version 1.4. Caltrans, June 2006. 
[7] C. B. Chadwell and Imbsen \& Associates. XTRACT - cross section analysis software for structural and earthquake engineering, 2002. http://www.imbsen.com/xtract.htm.

[8] C. B. Chadwell and R. A. Imbsen. XTRACT: A tool for axial force - ultimate curvature interaction. In Structures 2004 - Building On The Past Securing The Future, 2004.

[9] Hsiao-Lin Cheng. A Study of FRP Wrapped Reinforced Concrete Columns. PhD thesis, Purdue University, 2000.

[10] A. Considère. Résistance à la compression du beton armé et du beton fretté. In Experimental researches on reinforced concrete. McGraw Publishing Co., 1906. Translated by L. S. Moisseiff.

[11] M. N. Fardis and H. Khalili. FRP - encased concrete as a structural material. Magazine of Concrete Research, 34(No. 121):191 - 202, December 1982.

[12] T. G. Harmon and Wang H. H. Behavior of composite confined concrete under cyclic loading. Materials and Process Challenges: Aging Systems, Affordability, Alternative Ap, 41(II):975 - 989, 1996.

[13] G. F. Hawkins, N. R. Patel, G. L. Steckel, and M. Sultan. Failure analysis of highway bridge column composite overwraps. In Proceedings of the First International Conference on Composites in Infrastructure, pages 1126 - 1140. ICCI'96, 1996.

[14] C. E. Kurt. Concrete filled structural plastic columns. Journal of the Structural Division ASCE, 104(No. ST1):55 - 63, 1978.

[15] A. La Tegola and O. Manni. Experimental investigation on concrete confined by fiber 
reinforced polymer and comparison with theoretical model. In Fourth International Symposium on Fiber Reinforced Polymer Reinforcement for Reinforced Concrete Structures, pages $243-253,1999$.

[16] Laura De Lorenzis. A comparative study of models on confinement of concrete cylinders with FRP composites. Technical report, Chalmers University of Technology, 2001.

[17] J. B. Mander, M. J. N. Priestly, and R. Park. Theoretical stress-strain model for confined concrete. Journal of Structural Engineering, ASCE, 114(8):1804-1826, 1988.

[18] J. C. Mastrapa. Effect of bonded and unbonded construction on confinement with fiber composites. Master's thesis, University of Central Florida, Orlando, Fl, 1997.

[19] A. Mirmiran and M. Shahawy. A new concrete-filled FRP composite column. In Composites Part B: Engineering, volume 27B, pages 263 - 268, London, UK, 1996. Elsevier Science Ltd.

[20] A. Mirmiran and M. Shahawy. Behavior of concrete columns confined by fiber composites. Journal of Structural Engineering, ASCE, 123(No. 5):583 - 590, May 1997.

[21] K. Newman and J. B. Newman. Failure theories and design criteria for plain concrete. In Proceedings, International Engineering Materials Conference on Structure, Solid Mechanics and Engineering Design, Part 2, pages 963 - 995, New York, 1972. Wiley Interscience.

[22] T. Paulay and M. J. N. Priestley. Seismic Design of Reinforced Concrete and Masonry Buildings. Wiley-Interscience, New York, 1992. 
[23] S. Popovics. A numerical approach to the complete stress-strain curves for concrete. Cement and Concr. Res., 3(5):583 - 599, 1973.

[24] M. J. N. Priestley and G. M. Calvi. Seismic Design and Retrofit of Bridges. WileyInterscience, New York, 1996.

[25] F. E. Richart, A. Brandtzaeg, and R. L. Brown. A study of the failure of concrete under combined compressive stresses. Bulletin 185, University of Illinois Engineering Experimental Station, Champaign, Ill, 1928.

[26] F. E. Richart, A. Brandtzaeg, and R. L. Brown. The failure of plain and spirally reinforced concrete in compression. Bulletin 190, University of Illinois Engineering Experimental Station, Champaign, Ill, 1929.

[27] H. Saadatmanesh, M. R. Ehsani, and M. W. Li. Strength and ductility of concrete columns externally reinforced with fiber composite straps. ACI Structural Journal, 91(No. 4):434 - 447, July - August 1994.

[28] M. Saiidi Saiidi, Kandasamy Sureshkumar, and Claudia Pulido. Simple carbon-fiberreinforced-plastic-confined concrete model for moment-curvature analysis. Journal of Composites for Construction, pages 101 - 104, January/February 2005.

[29] Michael Samaan, Amir Mirmiran, and Mohsen Shahawy. Model of concrete confined by fiber composites. Journal of Structural Engineering, pages 1025 - 1031, September 1998.

[30] Mohsen Shahawy, Tom Beitelman, and Amir Mirmiran. Analysis and modeling of fiber wrapped columns and concrete-filled tubes. Technical report, Florida Department of 
Transportation Structural Research Center, 2007 E. Dirac Drive, Tallahassee, Fl 32310, June 1998.

[31] A. N. Talbot. Tests of concrete and reinforced concrete columns. Engineering Experiment Station Bulletin No. 10, University of Illinois, Urbana, Il, 1906.

[32] A. Zhang, T. Yamakawa, P. Zhong, and T. Oka. Experimental study on seismic performance of reinforced concrete columns retrofitted with composite-material jackets. In Fourth International Symposium on Fiber Reinforced Polymer Reinforcement for Reinforced Concrete Structures, pages 269 - 278, 1999. 


\section{APPENDIX A}

\section{Parameter Study Figures}




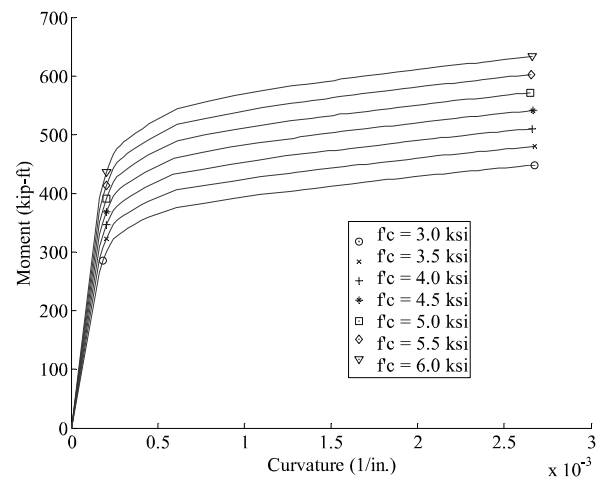

(a) moment-curvature

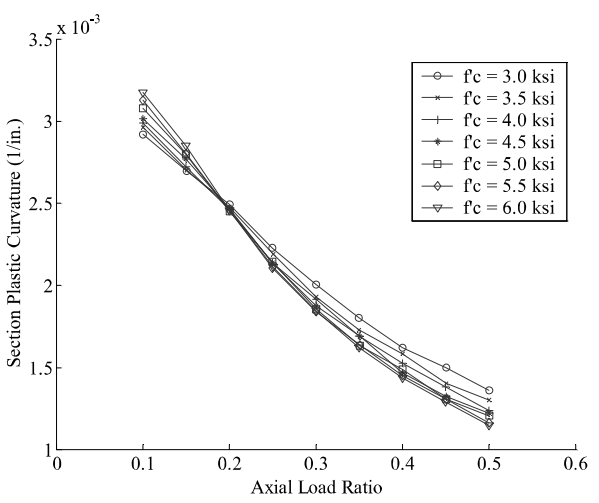

(c) section plastic curvature

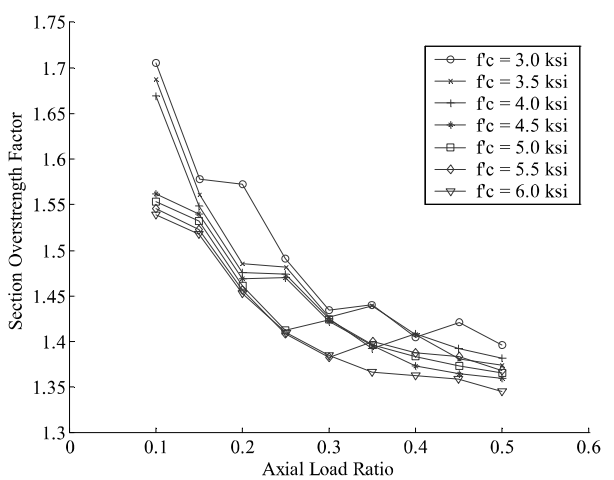

(e) section overstrength factor

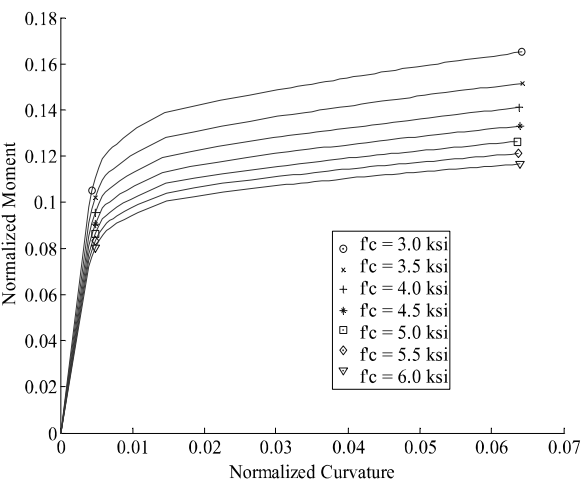

(b) normalized moment-curvature

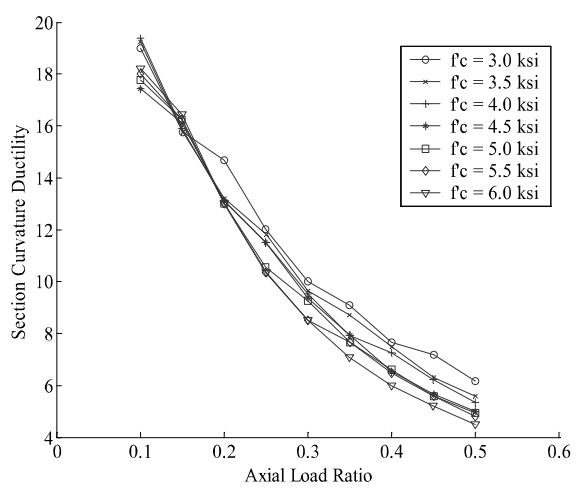

(d) section curvature ductility

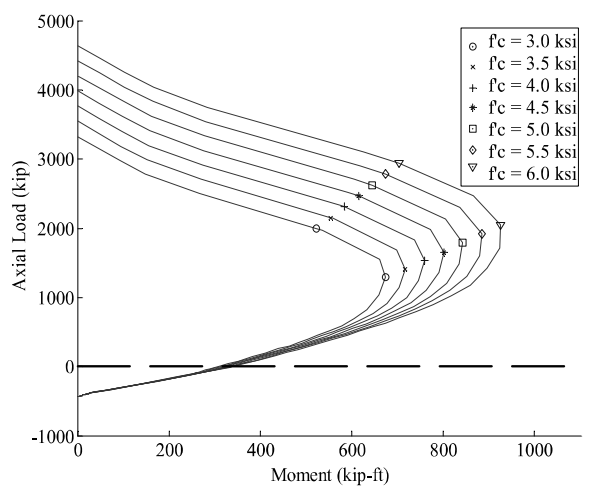

(f) axial load-moment interaction

FIGURE A.1: $f_{c}^{\prime}$ Parameter Study for FRP Confined Concrete 


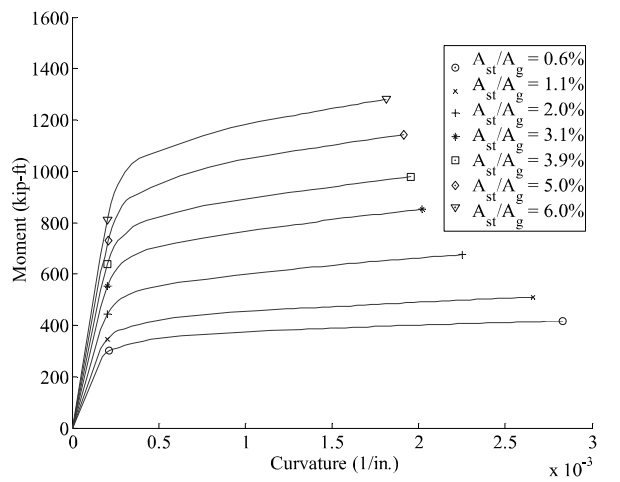

(a) moment-curvature

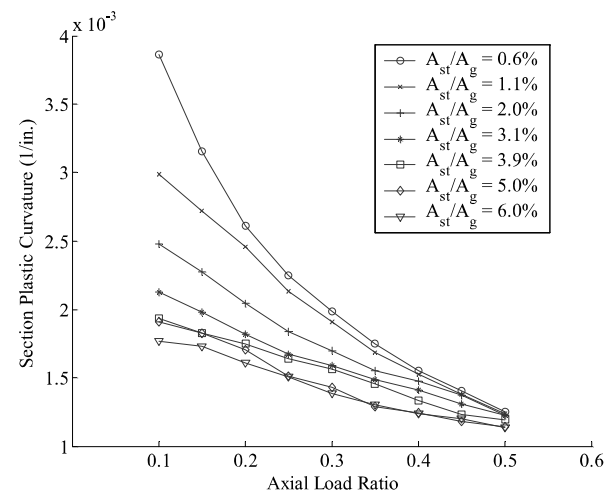

(c) section plastic curvature

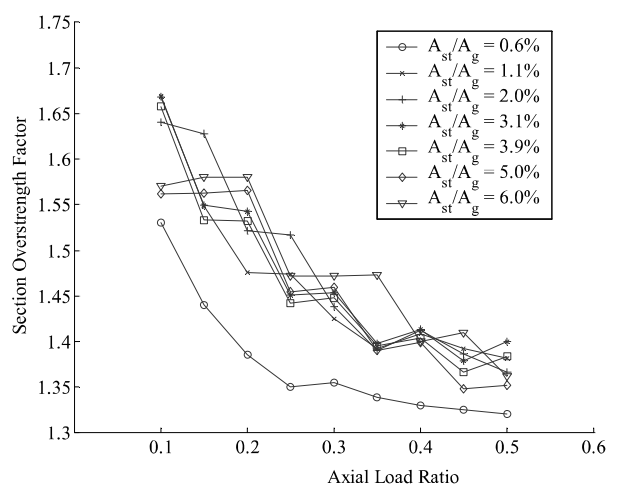

(e) section overstrength factor

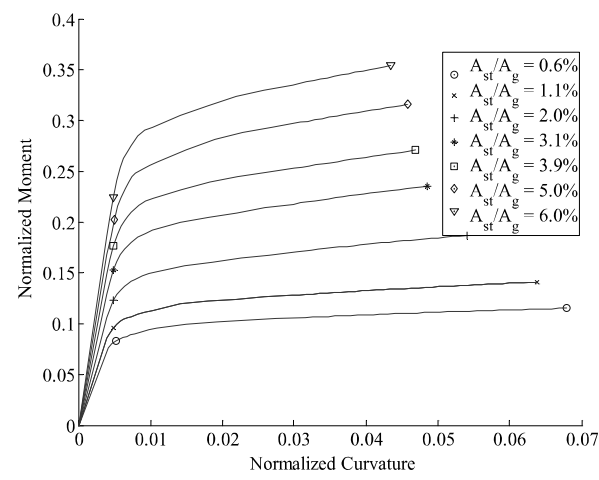

(b) normalized moment-curvature

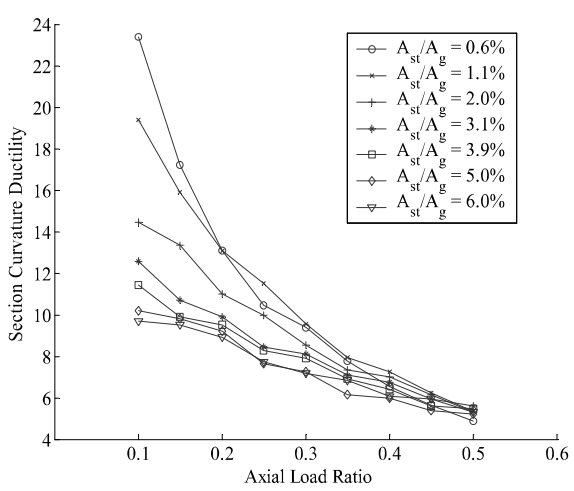

(d) section curvature ductility

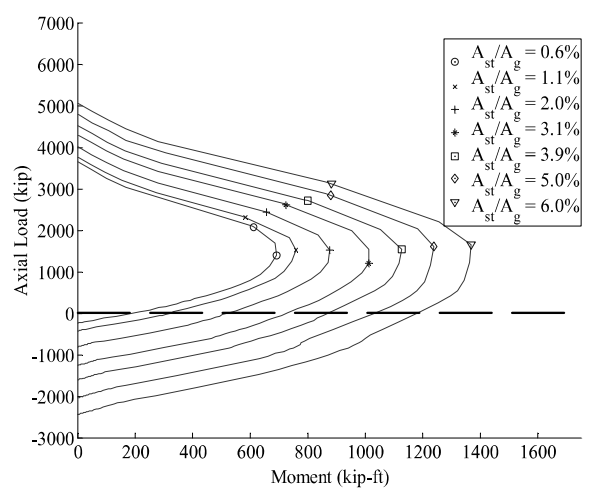

(f) axial load-moment interaction

FIGURE A.2: $\frac{A_{s t}}{A_{g}}$ Parameter Study for FRP Confined Concrete 


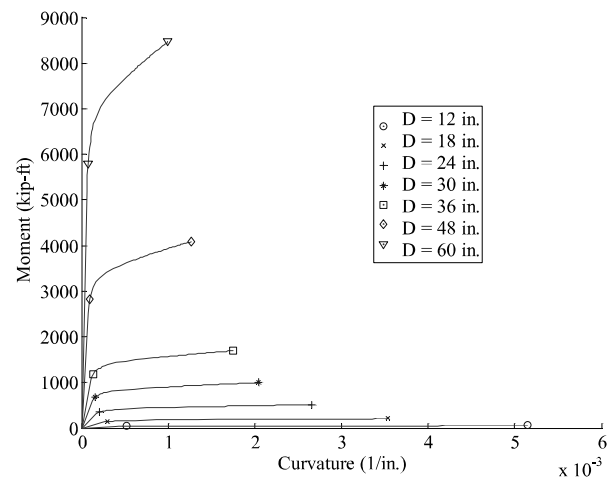

(a) moment-curvature

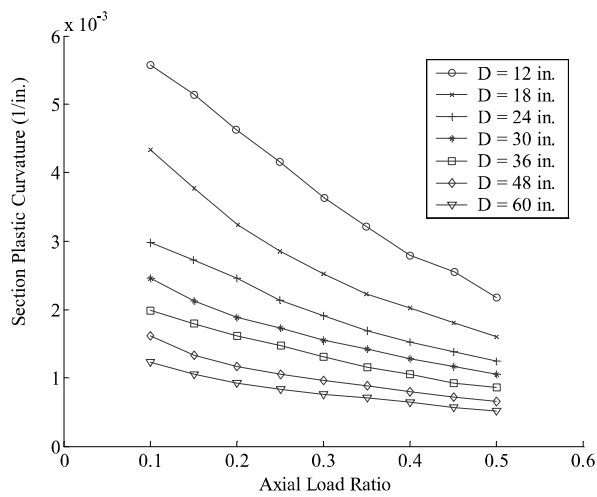

(c) section plastic curvature

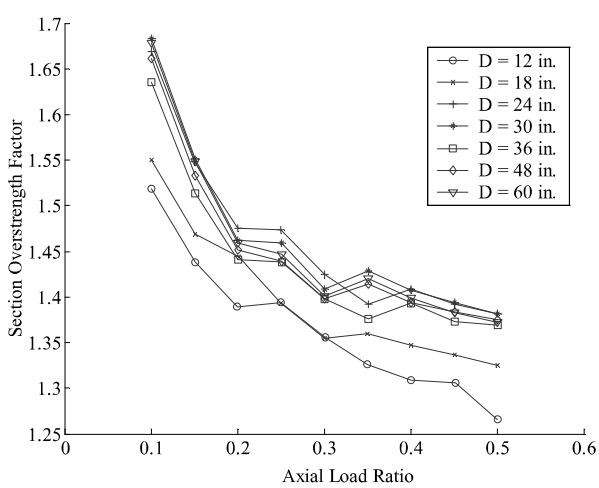

(e) section overstrength factor

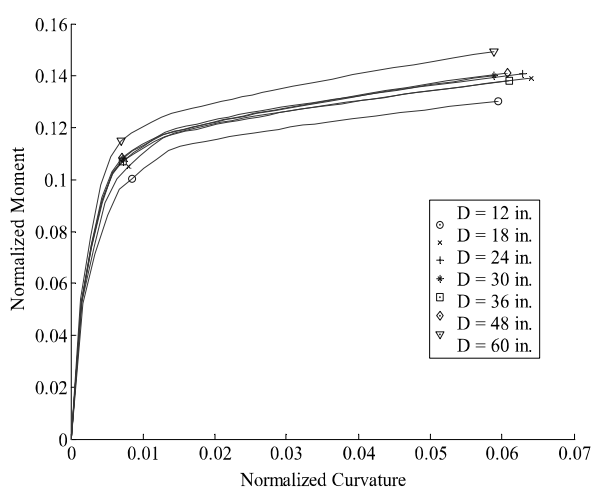

(b) normalized moment-curvature

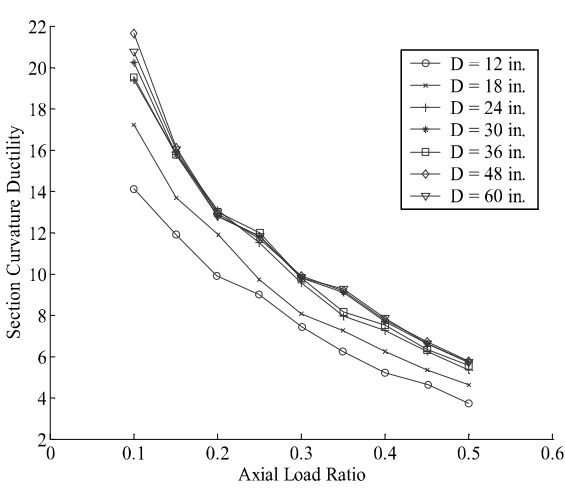

(d) section curvature ductility

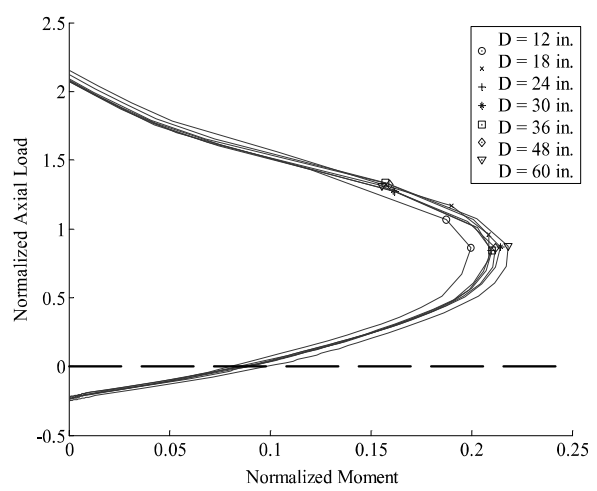

(f) normalized axial load-moment interaction

FIGURE A.3: $D$ Parameter Study for FRP Confined Concrete 


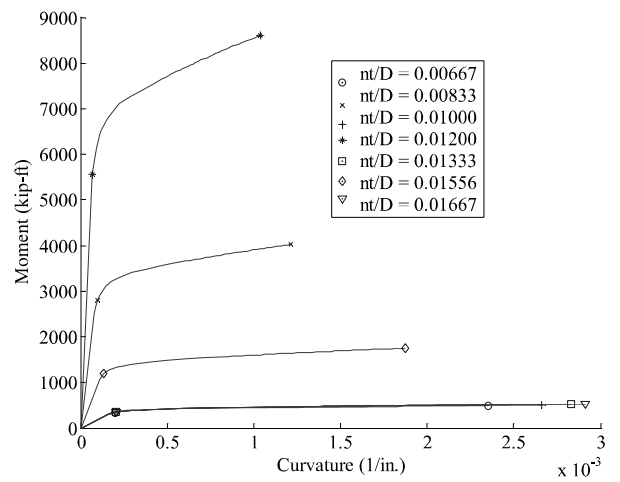

(a) moment-curvature

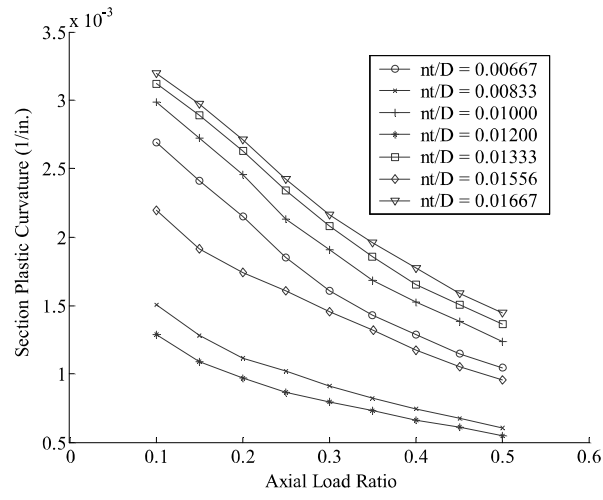

(c) section plastic curvature

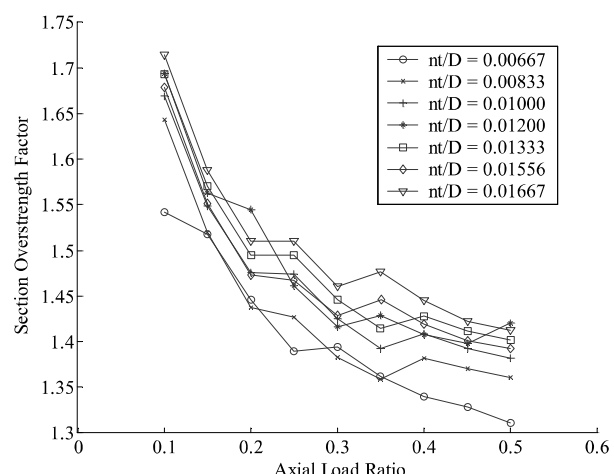

(e) section overstrength factor

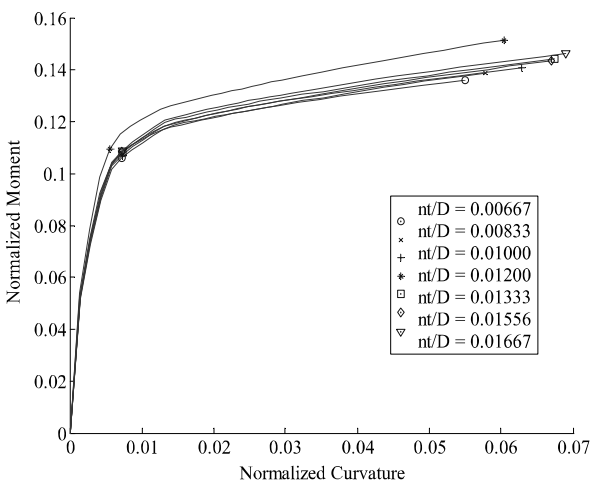

(b) normalized moment-curvature

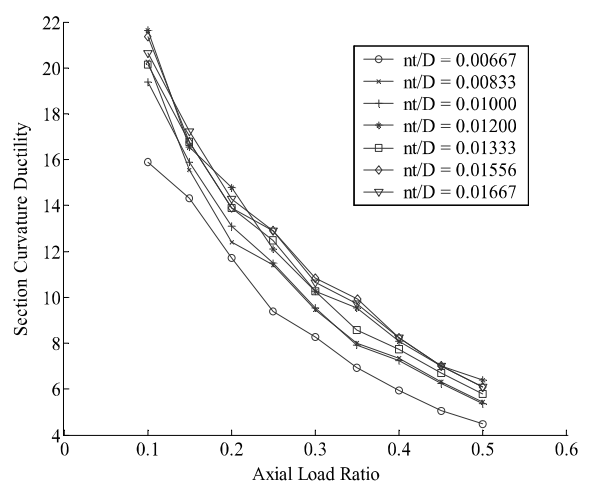

(d) section curvature ductility

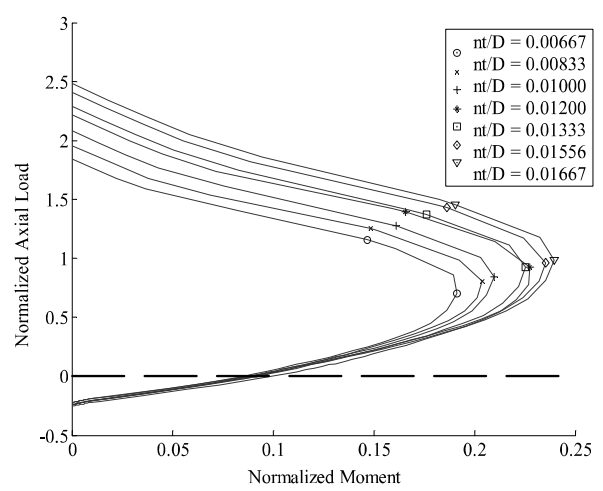

(f) normalized axial load-moment interaction

FIGURE A.4: $\frac{n t}{D}$ Parameter Study for FRP Confined Concrete 


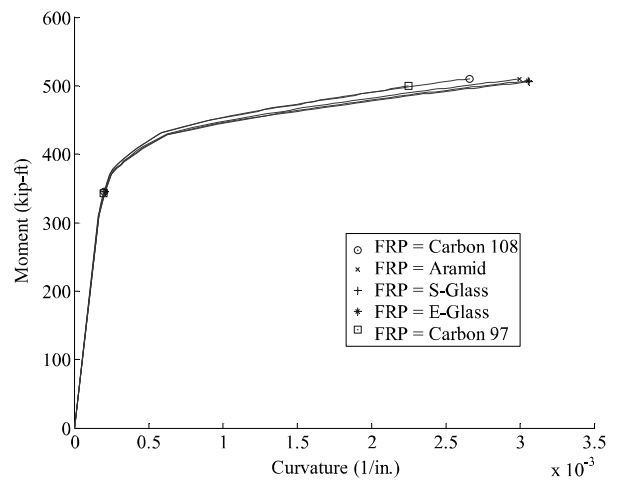

(a) moment-curvature

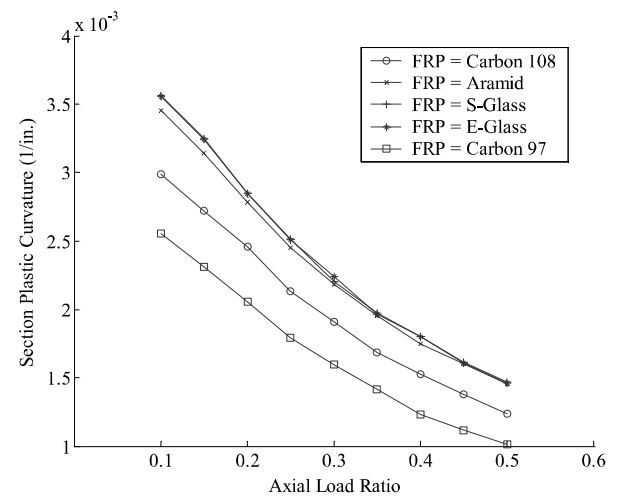

(c) section plastic curvature

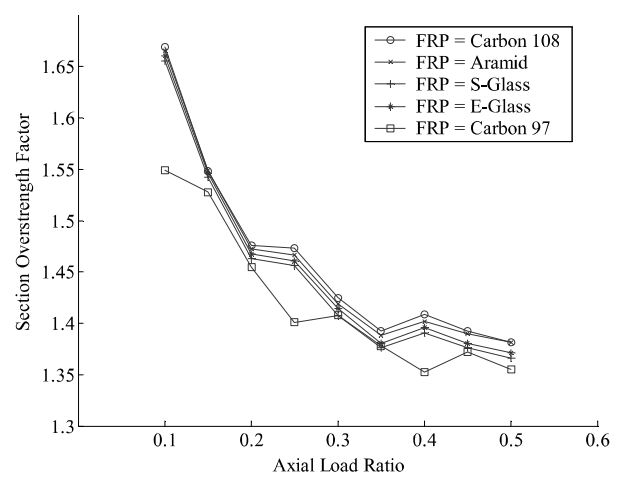

(e) section overstrength factor

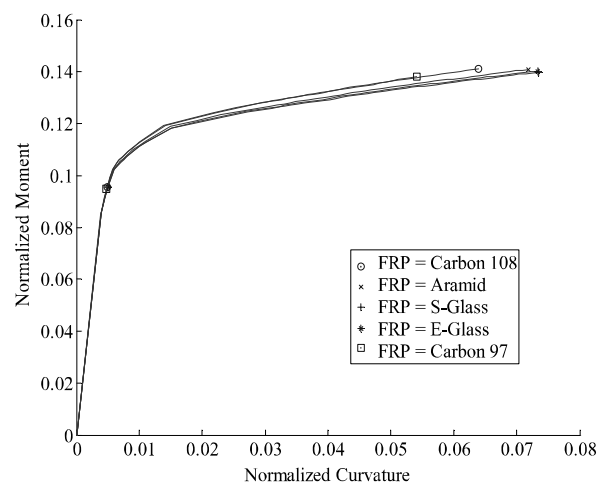

(b) normalized moment-curvature

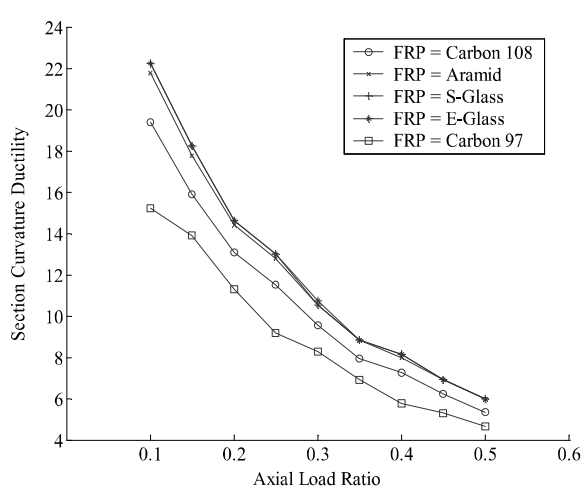

(d) section curvature ductility

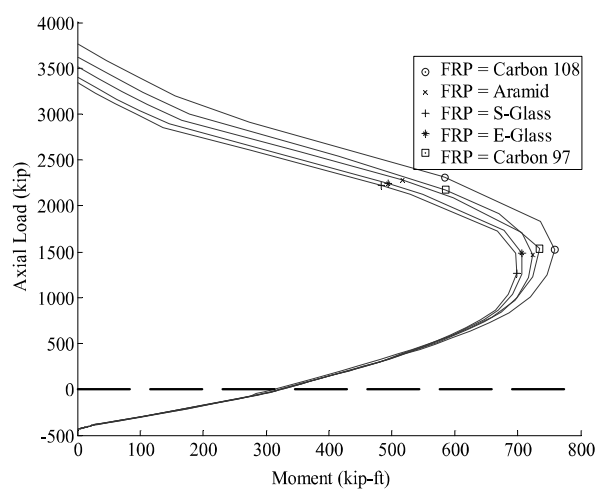

(f) axial load-moment interaction

FIGURE A.5: System Parameter Study for FRP Confined Concrete 


\section{APPENDIX B}

\section{MC and PM Interaction Software}

\section{B.1 Driver Script for MC and PM Interaction Analyses}

The following script calls the variable input scripts and the necessary functions.

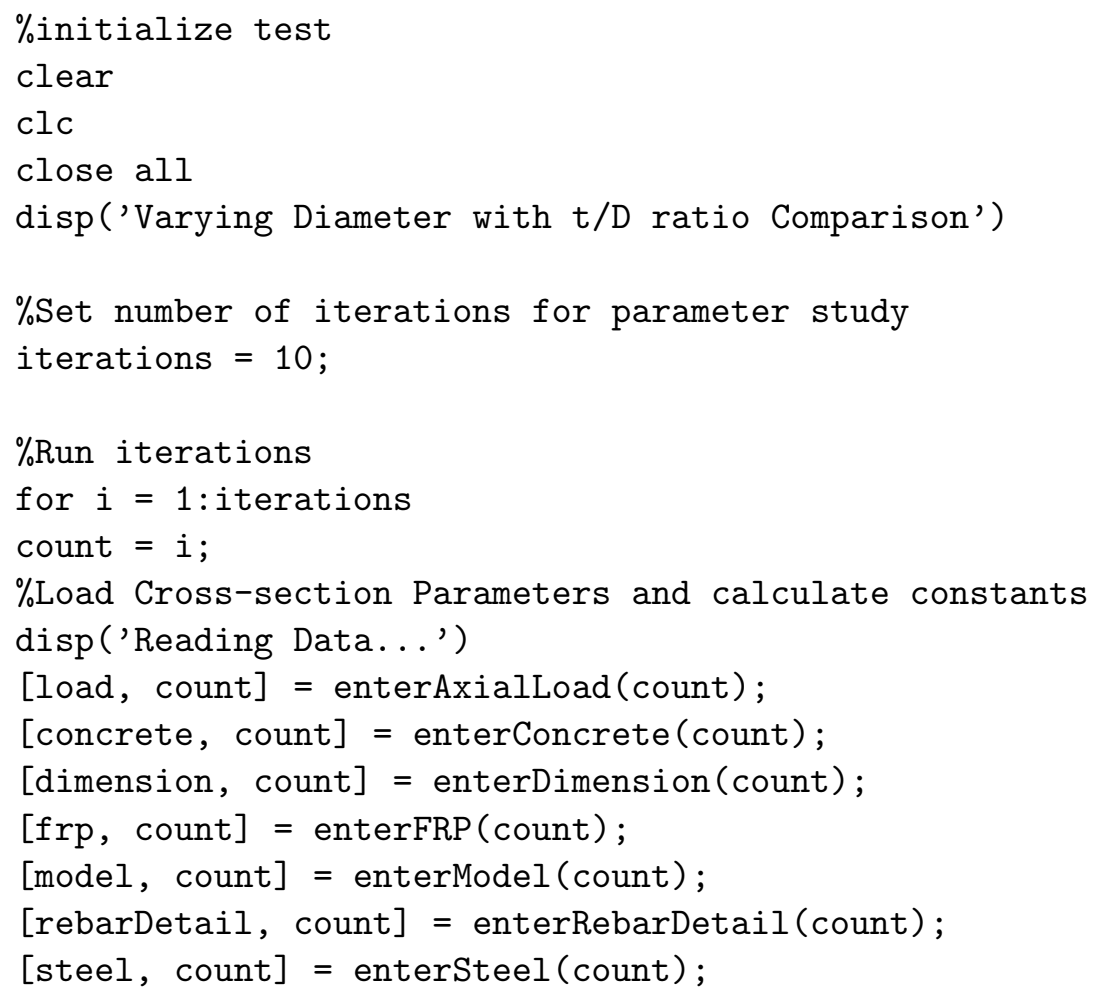




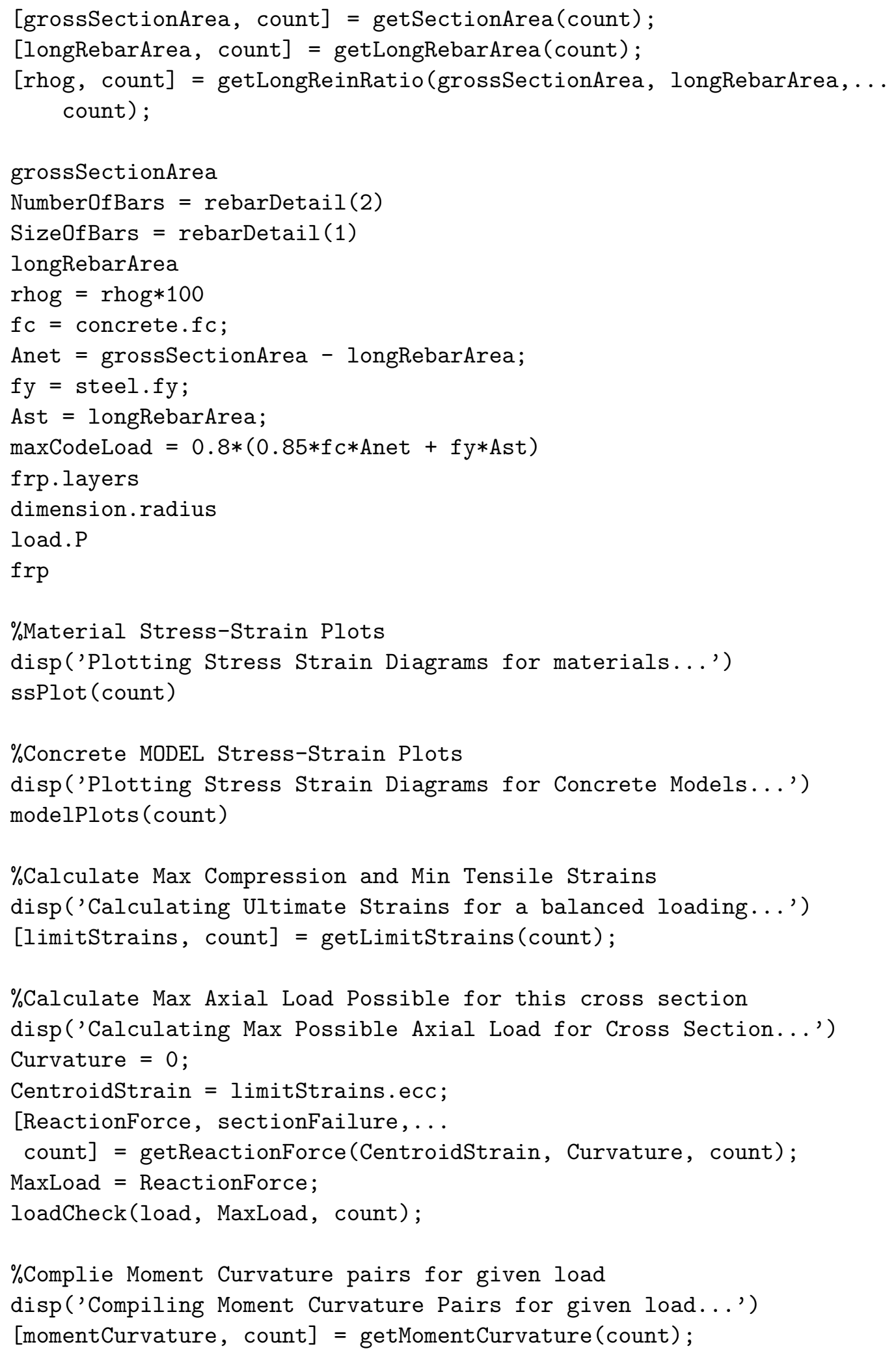




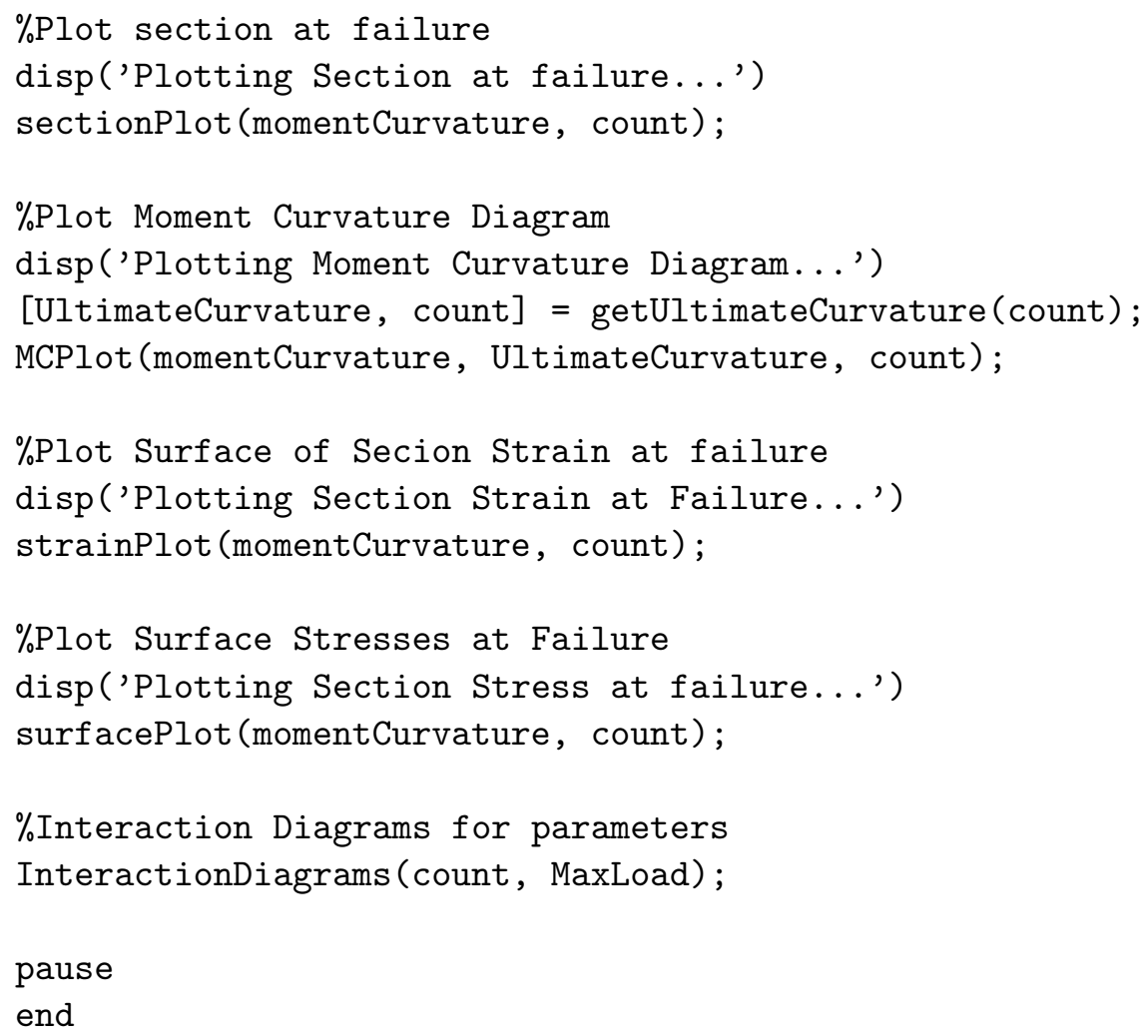

\section{B.2 Axial Load Input Script}

This script is where the applied axial load(s) for the MC and PM Interaction Analyses are entered.

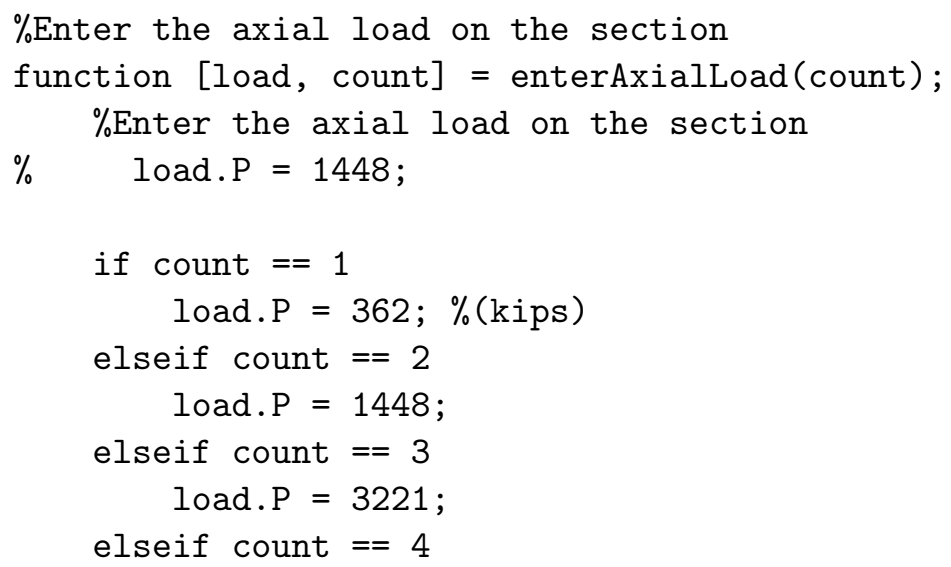




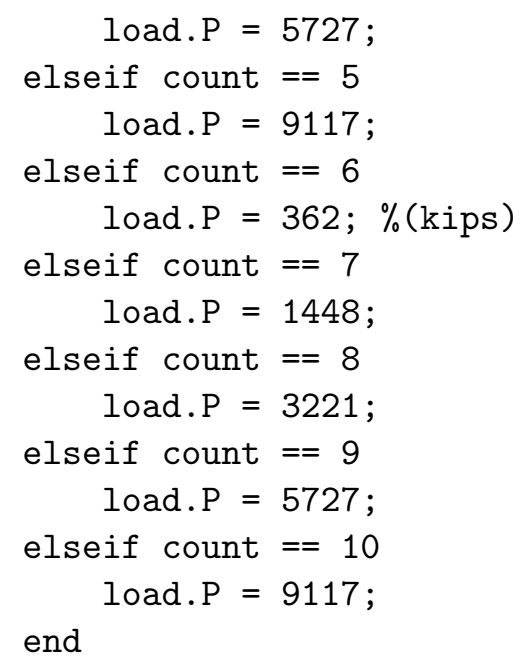

\section{B.3 Unconfined Concrete Material Properties Input Script}

This script is where the unconfined concrete material properties for the MC and

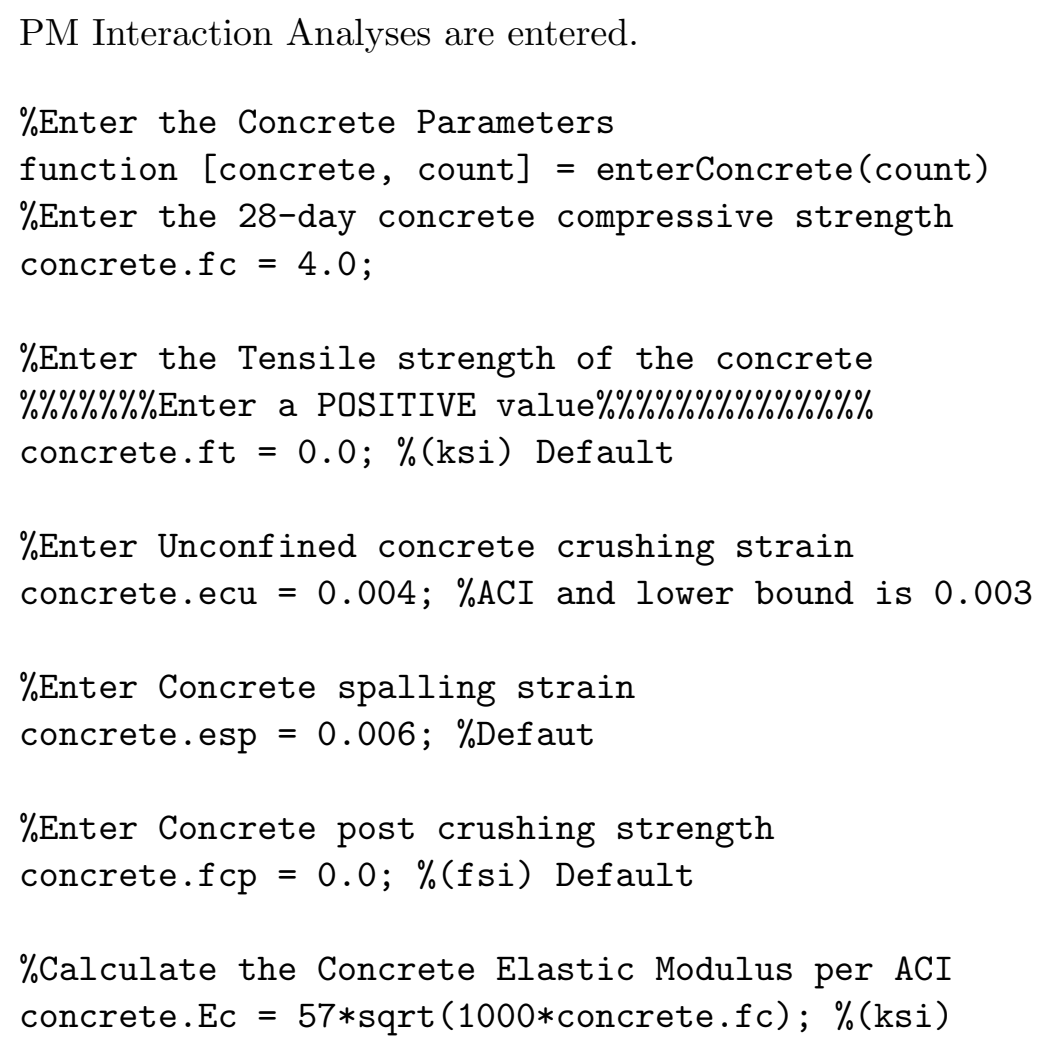


$\%$ Calculate the strain at the concrete tensile strength concrete.et $=-$ concrete.ft/concrete.Ec;

\section{B.4 Cross Section Diameter Input Script}

This script is where the cross section diameter(s) for the MC and PM Interaction

Analyses are entered.

\%Input The Section Dimensions

function [dimension, count] = enterDimension (count)

$\%$ Enter the Crossesection type

$\% 1=$ Round

$\% 2$ = Rectangular

dimension.type $=1$;

if dimension.type $==2$

$\%$ For rectangular sections enter a corner radius (in)

$\%$ Corner Radius must be greater than 0.5 inches...

for FRP wraps

dimension. CornerRadius $=2$;

$\%$ For rectangular sections enter the dimensions

$\%$ Enter the section width (in)

dimension.width $=20$;

$\%$ Enter the section height (in)

dimension.height $=20$;

if dimension.CornerRadius > dimension.width/2

error('The Corner Radius can not be...

bigger than the width of the section')

end

if dimension.CornerRadius > dimension.height/2

error('The Corner Radius can not be bigger... then the height of the section')

end

else

$\%$ For round sections enter the radius (in)

$\% \quad$ dimension.radius $=12 ;$

if count $==1$

dimension.radius $=6$;

elseif count $==2$

dimension.radius $=12$;

elseif count $==3$ 


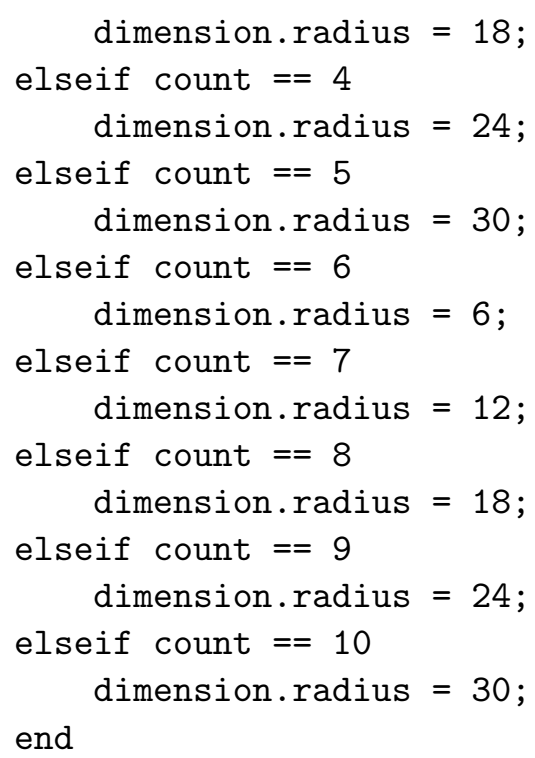

\section{B.5 FRP System Parameters Input Script}

This script is where the FRP system parameters for the MC and PM Interaction Analyses are entered.

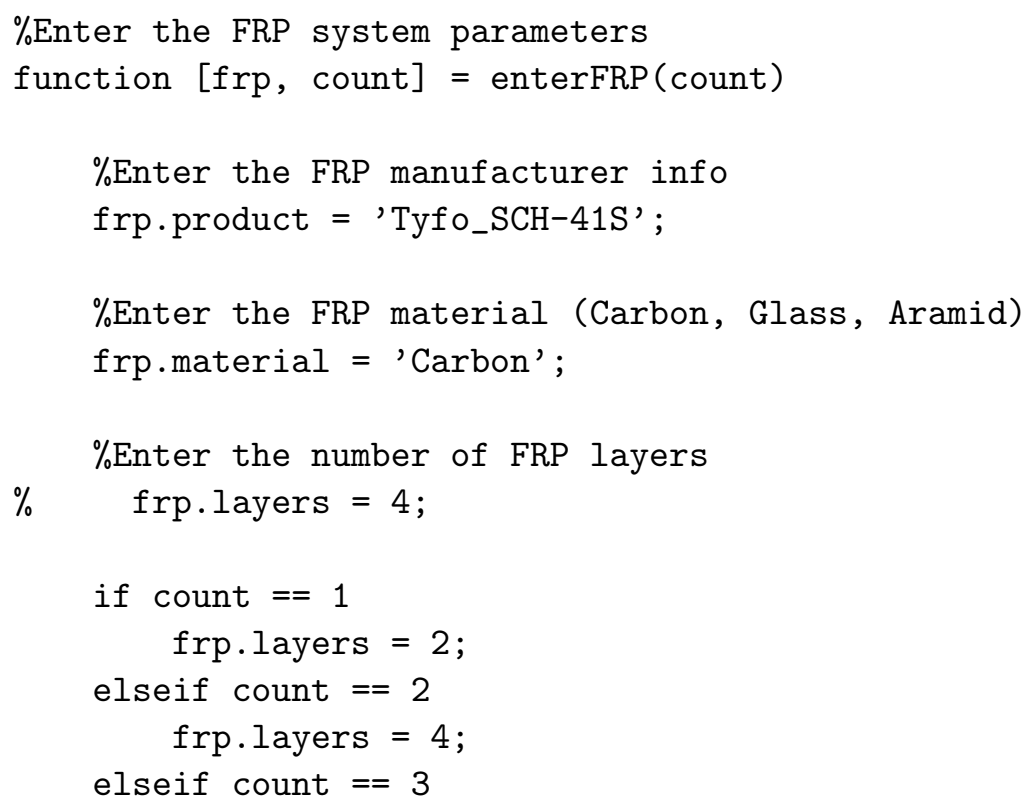




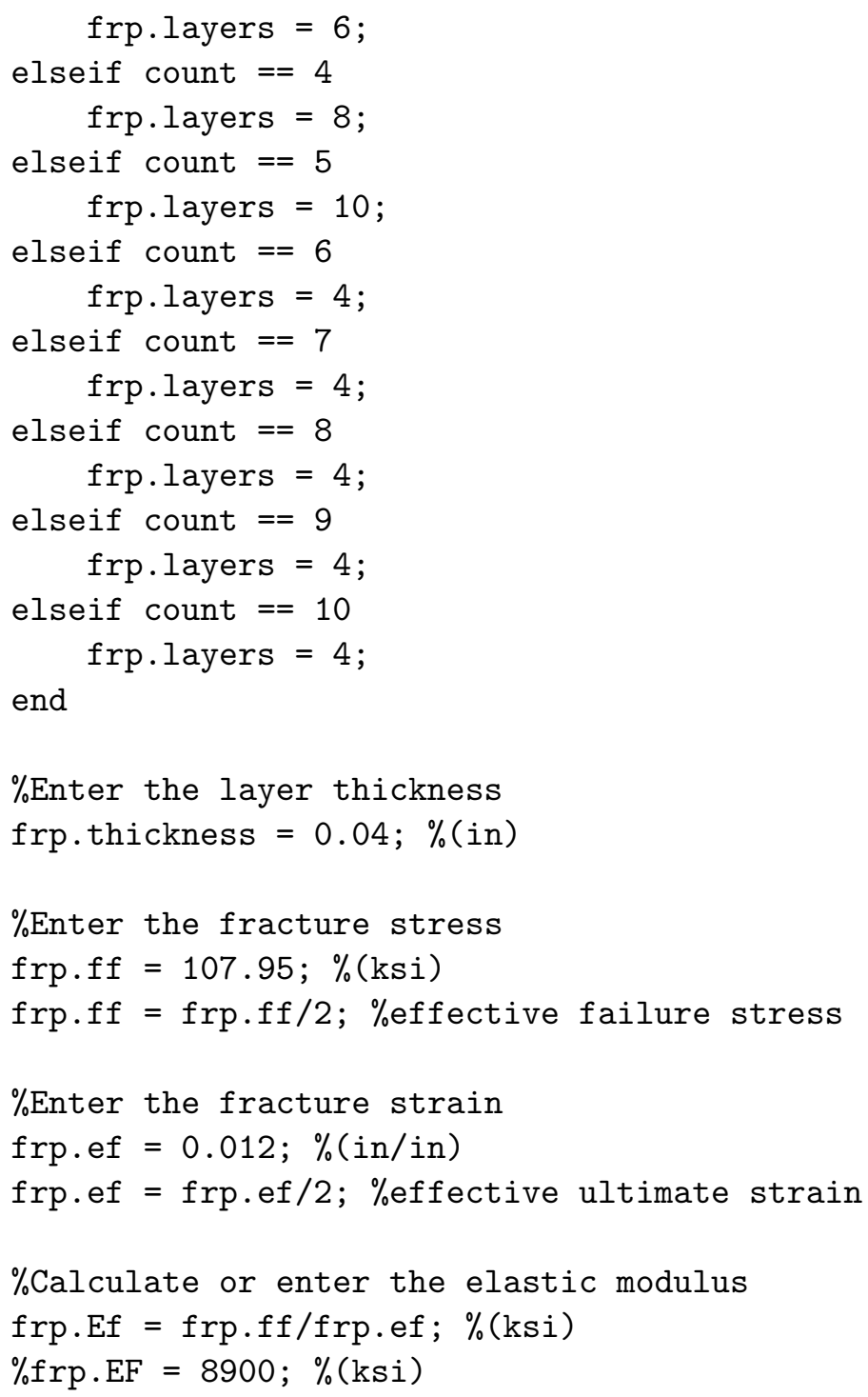

\section{B.6 Material Model Selection Script}

This script is where the material models for the MC and PM Interaction Analyses are selected.

$\%$ Select the material models used function [model, count] = enterModel (count);

format short

$\%$ FRP Confined Concrete Models 


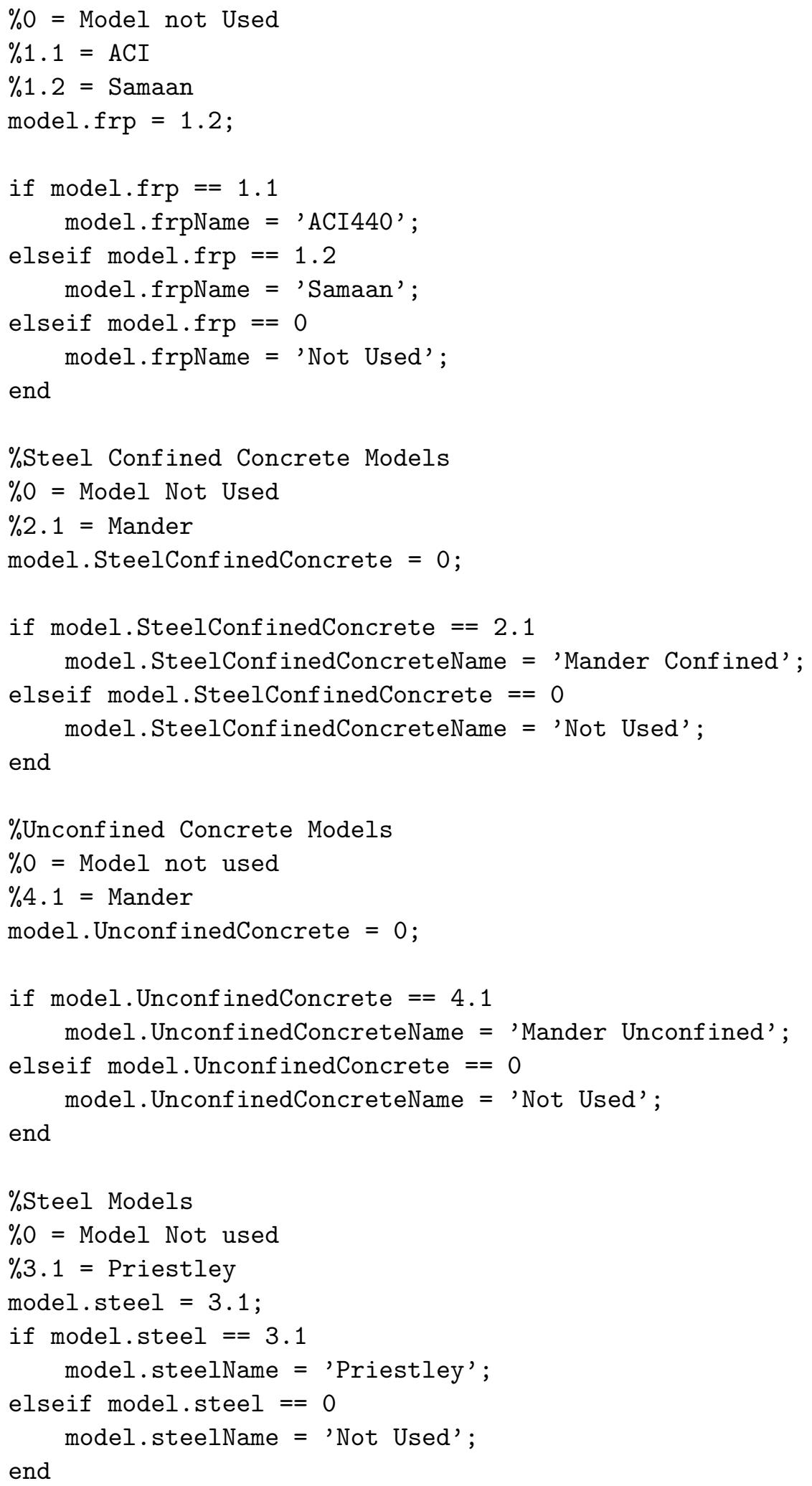




\section{B.7 Longitudinal Rebar Input Script}

This script is where the longitudinal reinforcement detail(s) for the MC and PM

Interaction Analyses are entered.

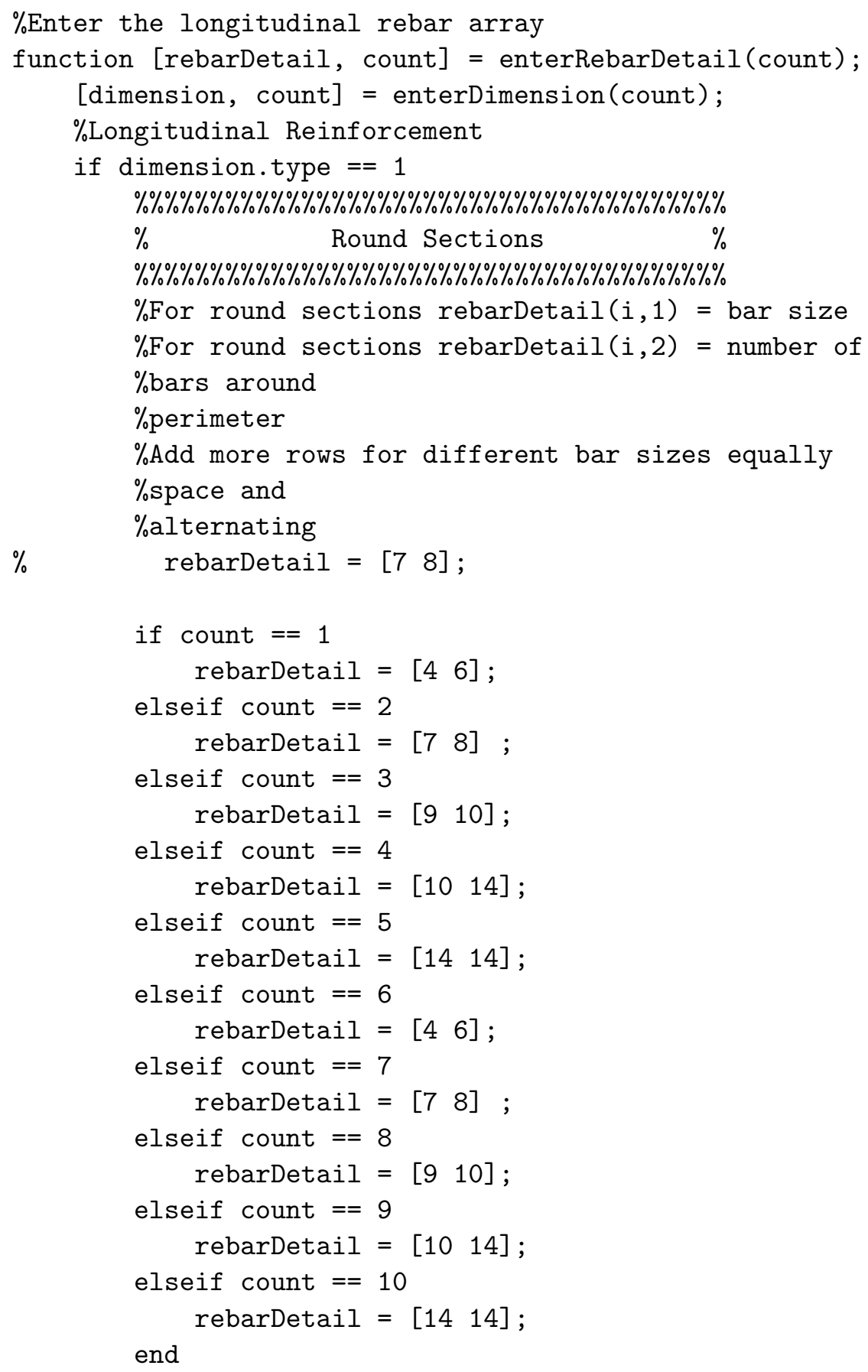




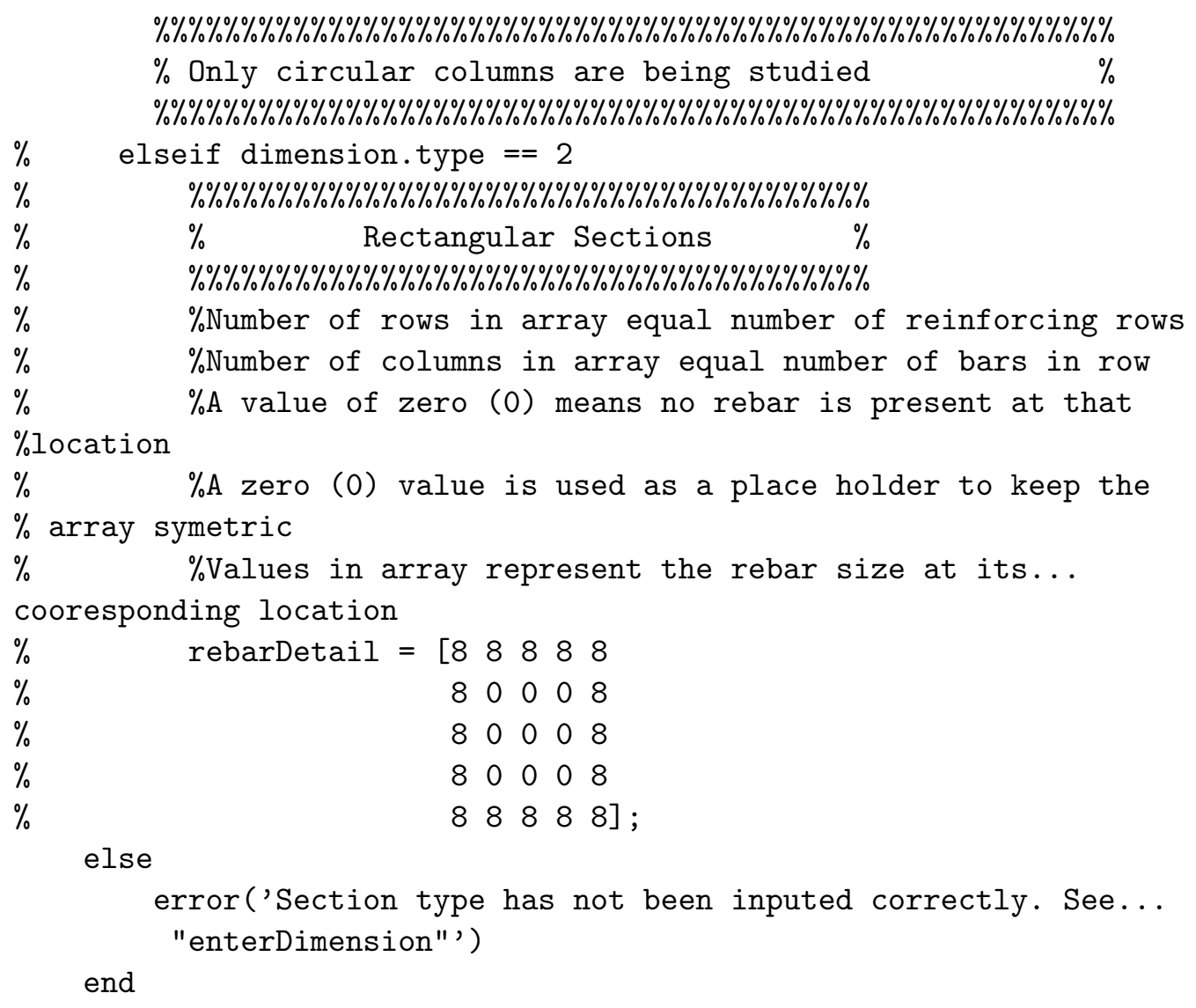

\section{B.8 Reinforcing Steel Material Properties Input Script}

This script is where the reinforcing steel material properties for the MC and PM Interaction Analyses are entered.

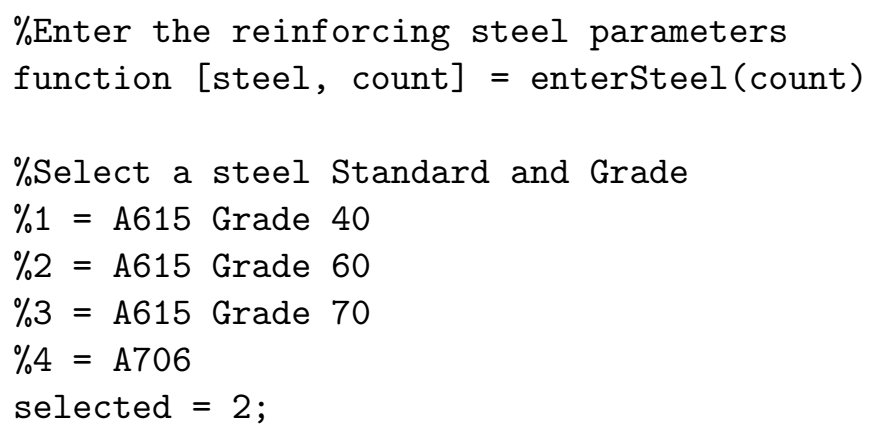




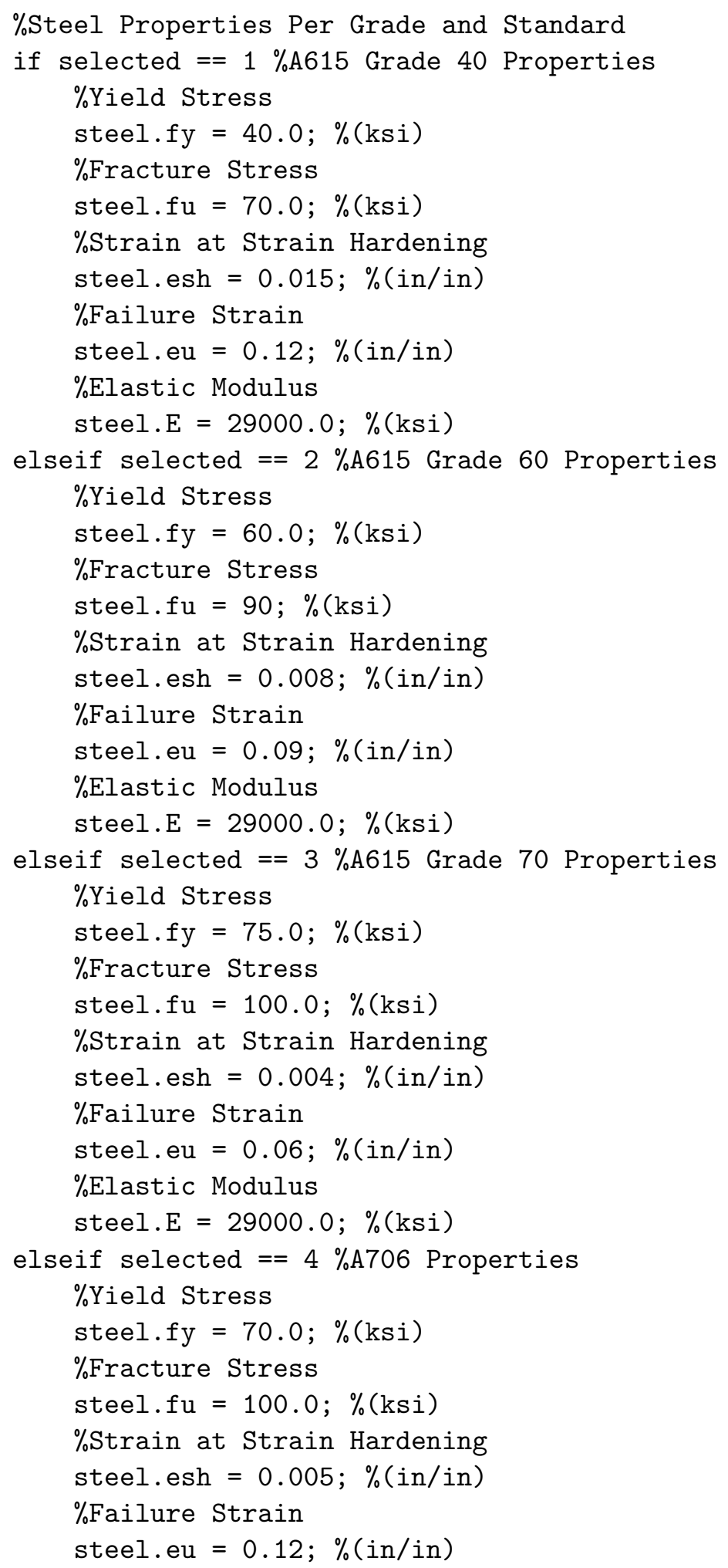




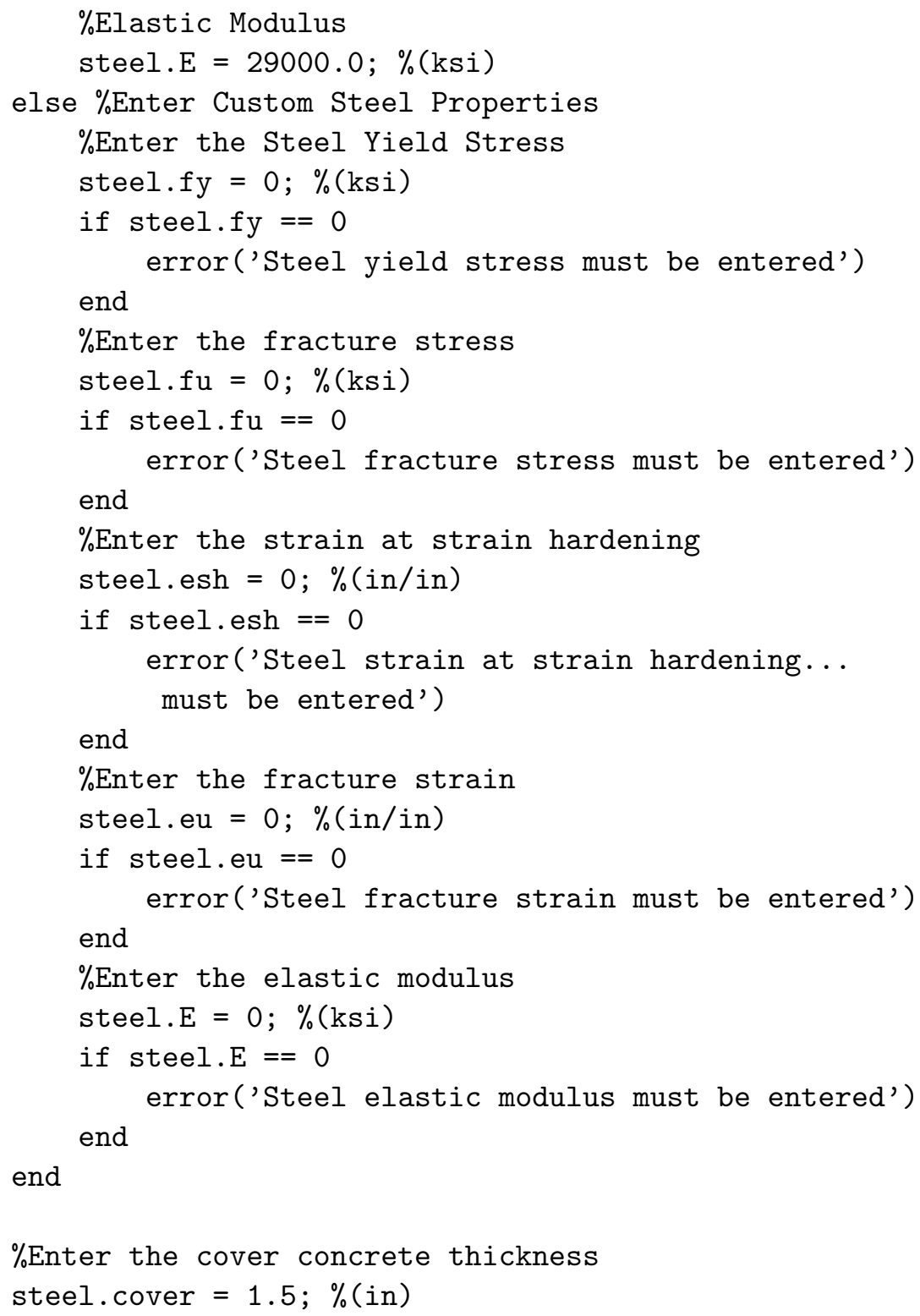

\section{B.9 Transverse Reinforcement Detail Input Script}

This script is where the transverse reinforcement detail for the MC and PM Interaction Analyses are entered.

$\%$ Enter the transverse rebar array function [transverseRebarDetail,... 


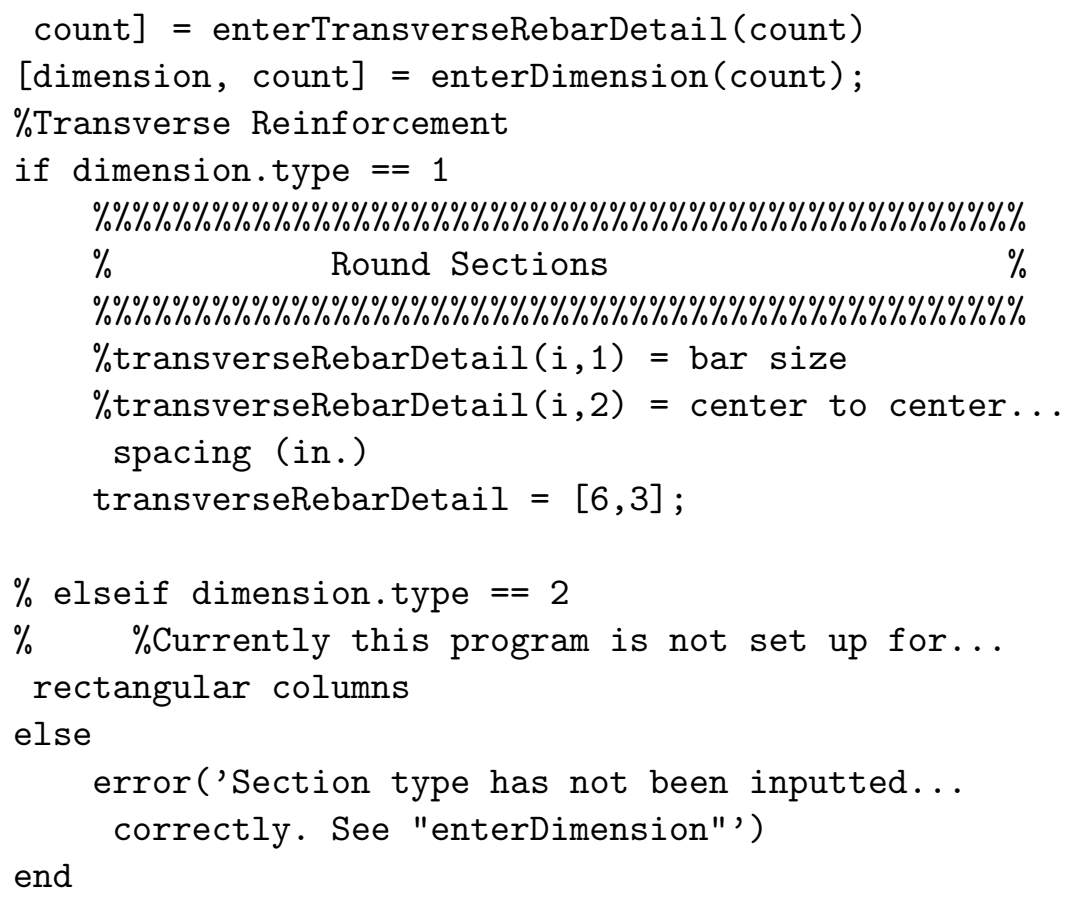

\section{B.10 Layer Centroid Strain for Moment-Curvature Analyses}

This function calculates the centroid strains of cross section layers for the Moment-

Curvature Analyses.

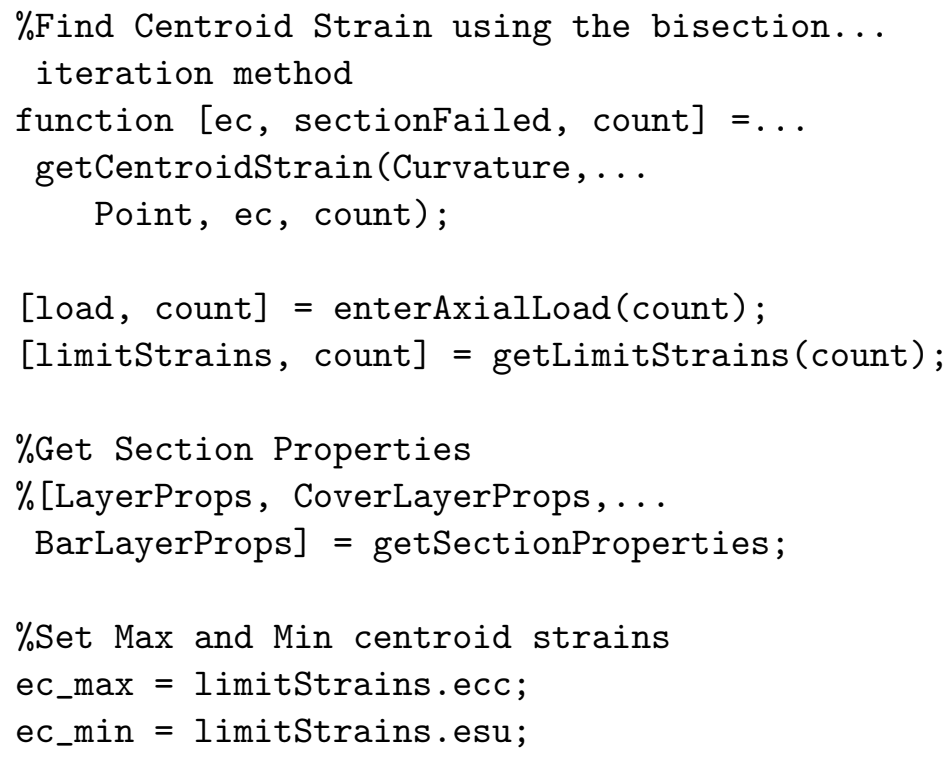




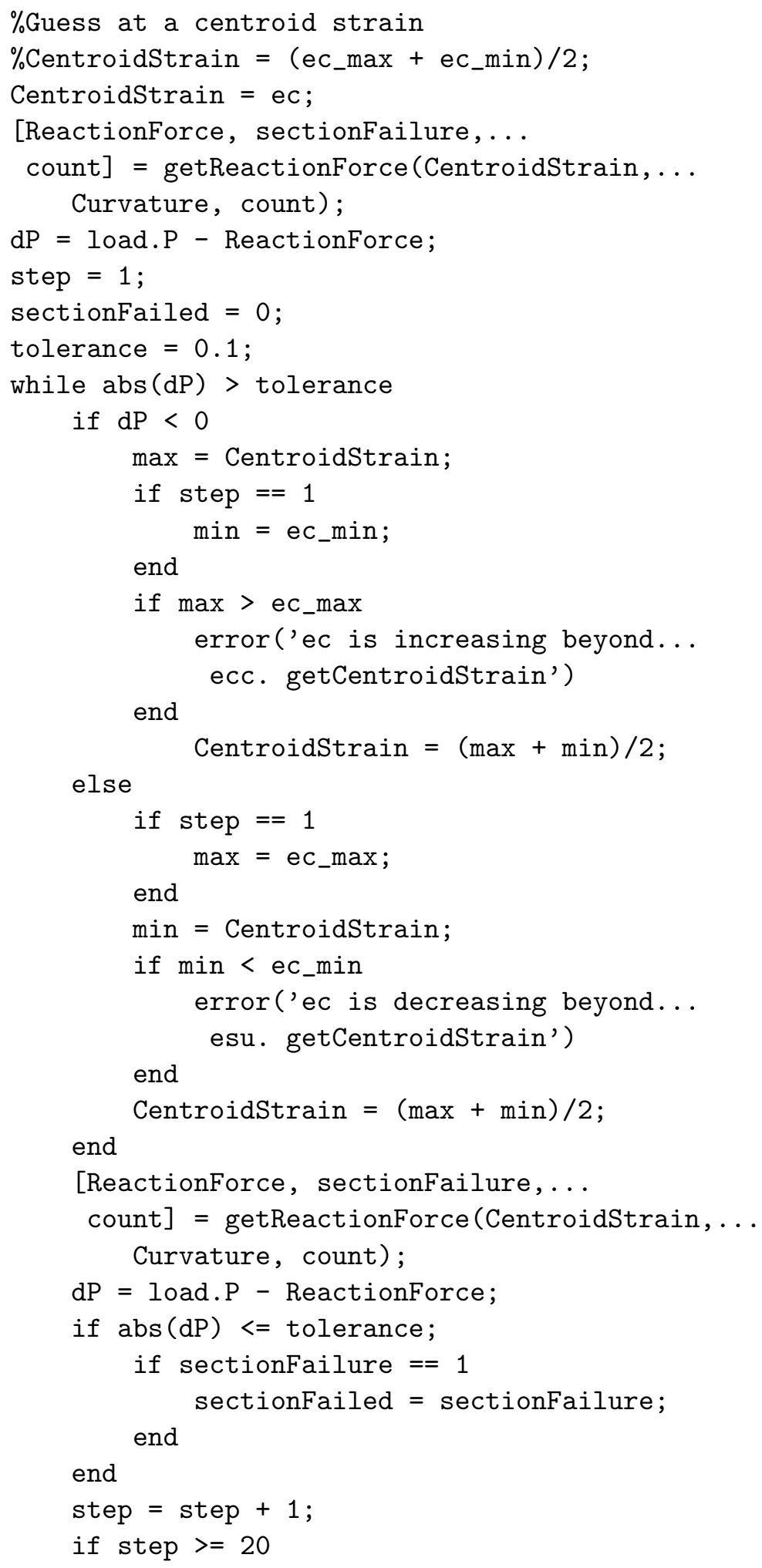




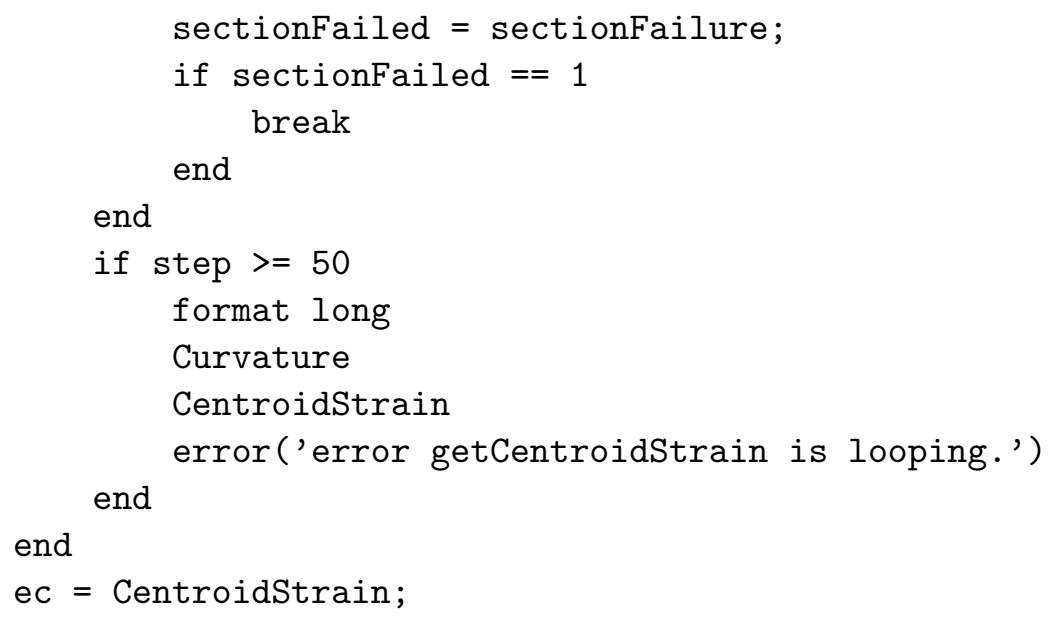

\section{B.11 Layer Centrior Strain for PM Interaction Analyses}

This function calculates the centroid strains of cross section layers for the PM Interaction Analyses.

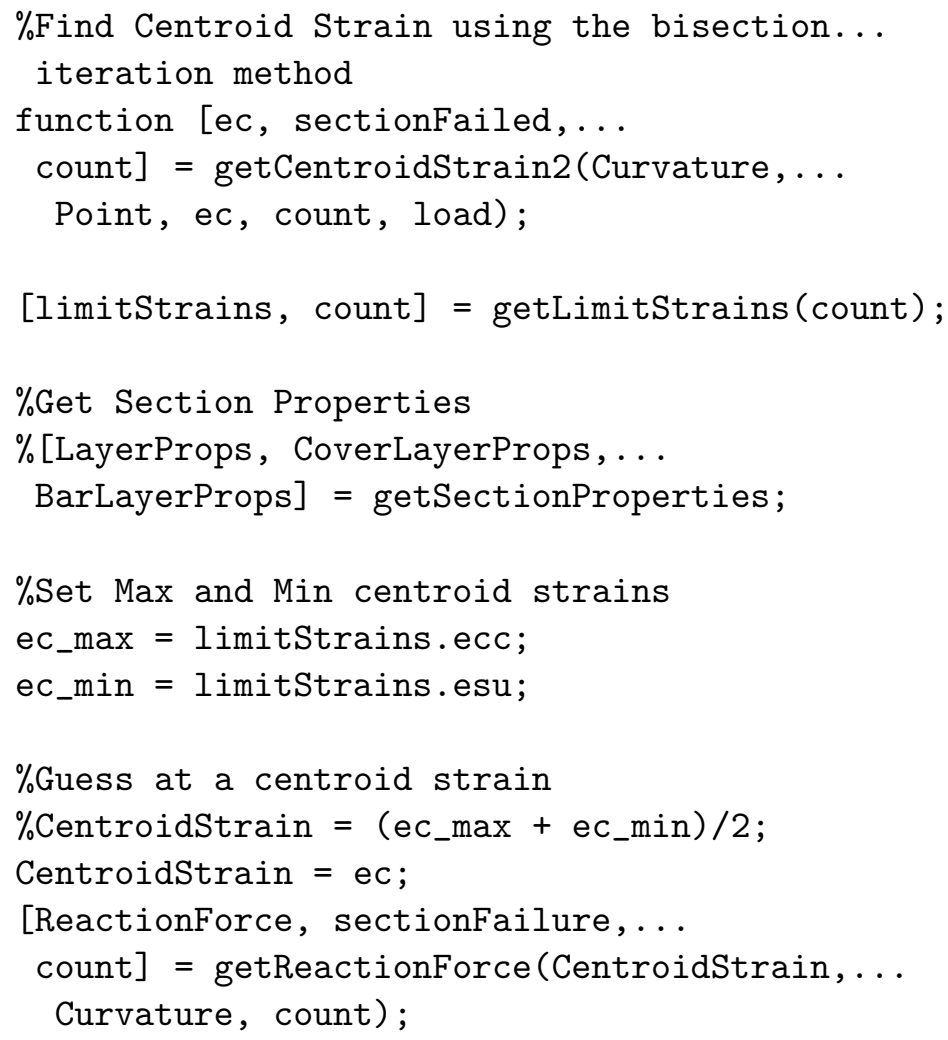




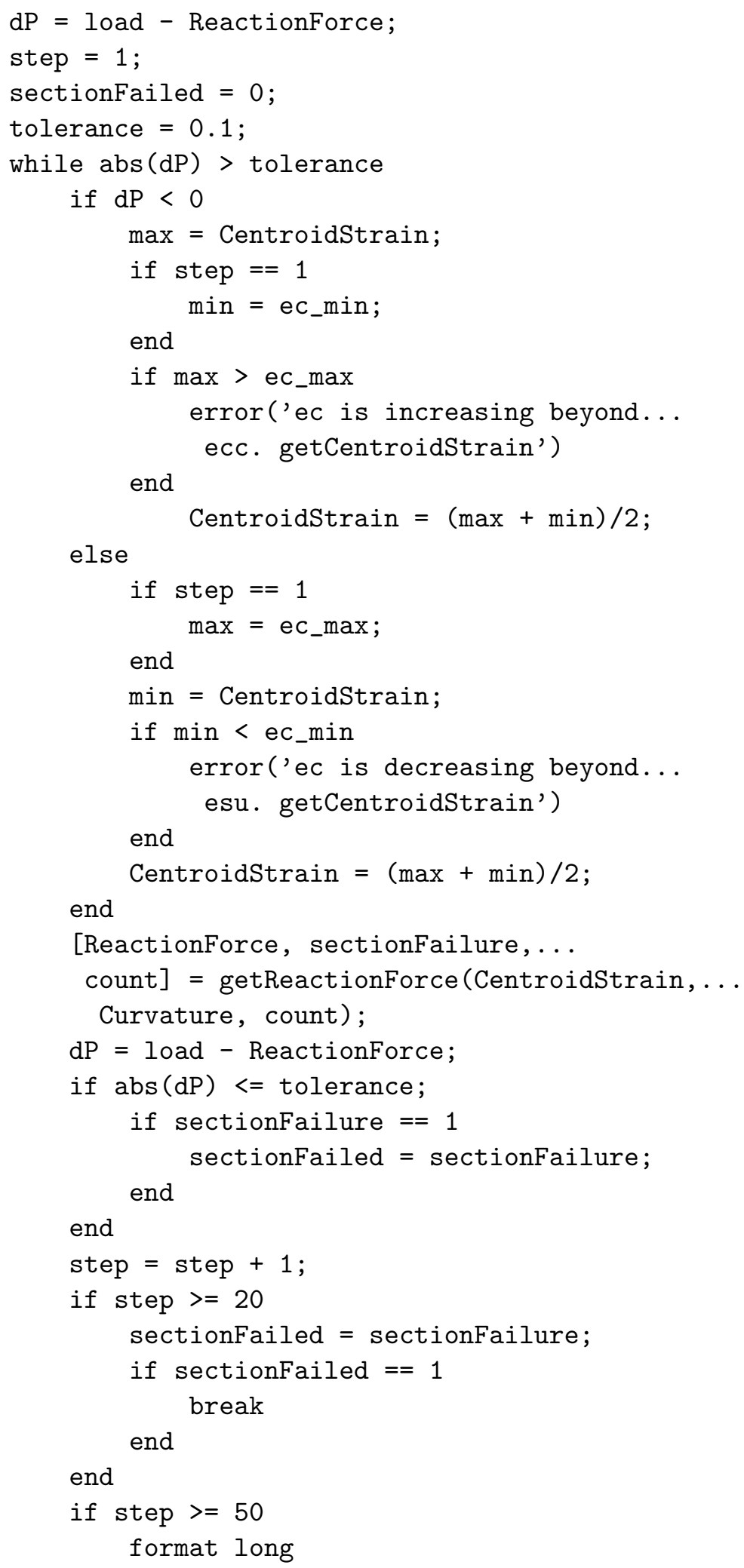




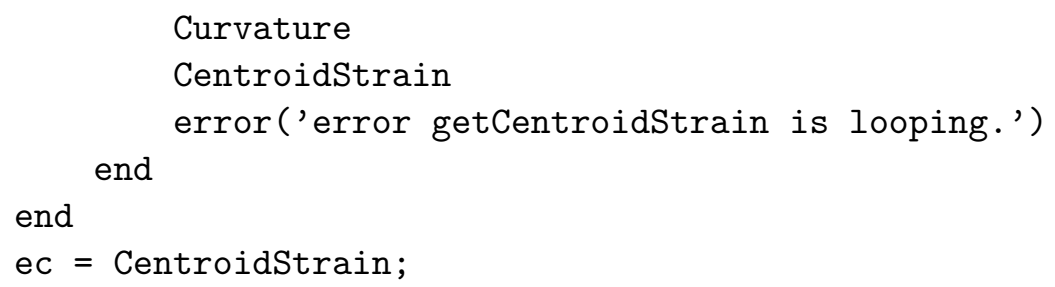

\section{B.12 Confined Concrete Stress}

This function calculates the stress in confined concrete for a given strain.

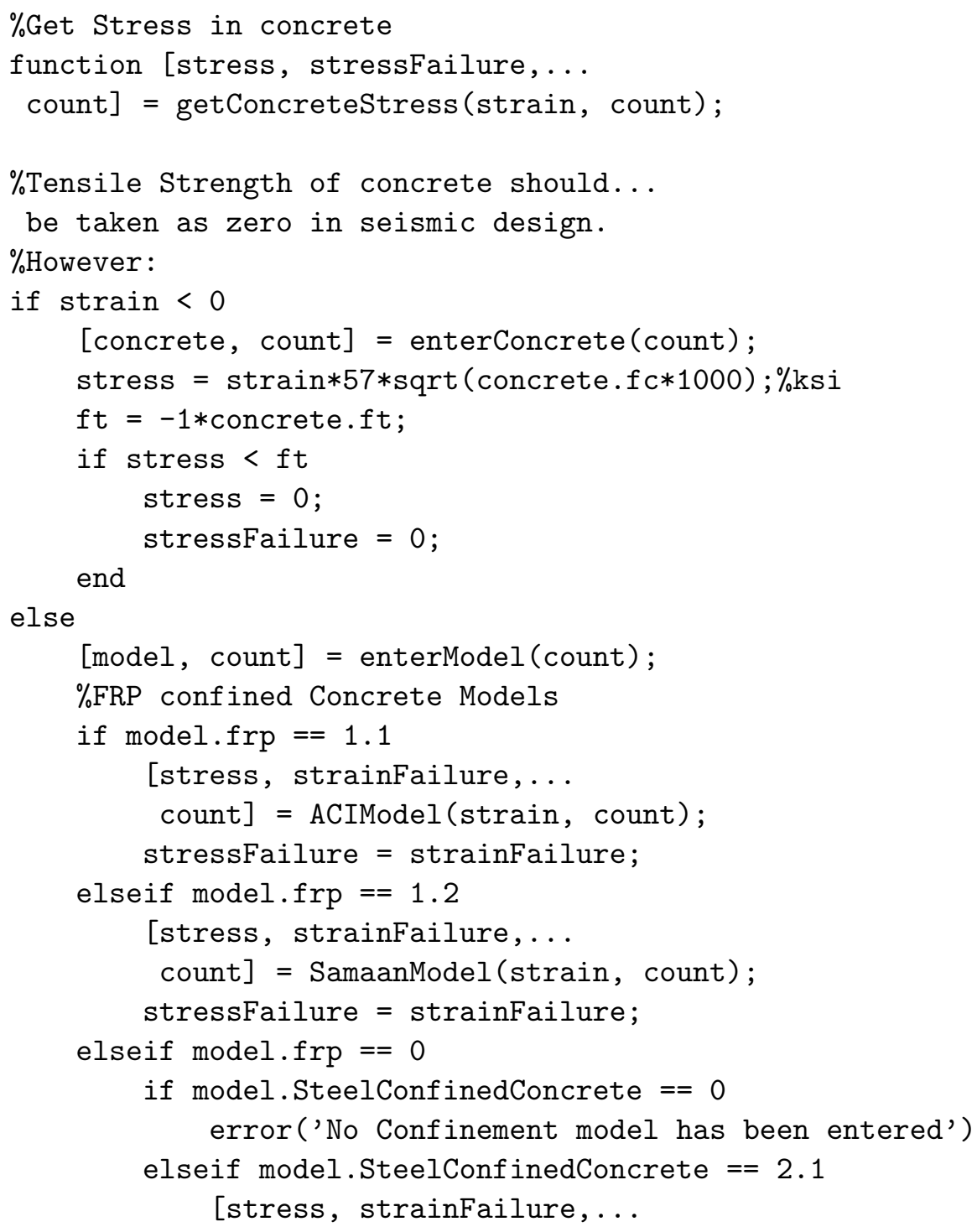




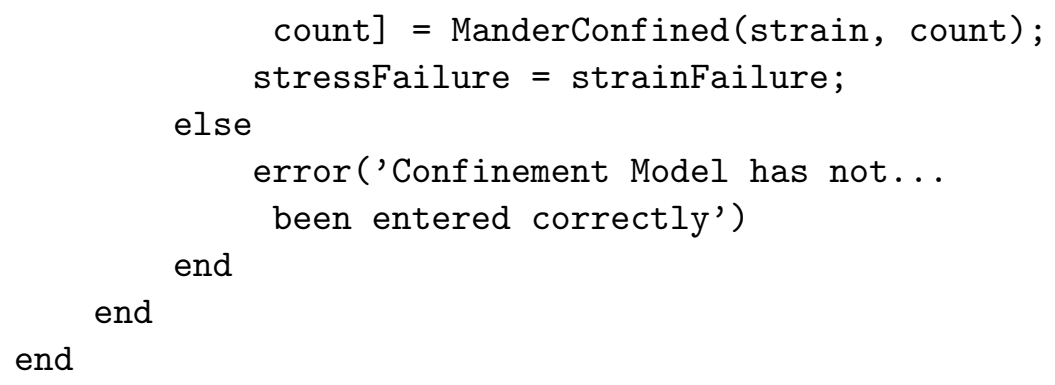

\section{B.13 Unconfined Concrete Stress}

This function calculates the stress in unconfined concrete for a given strain.

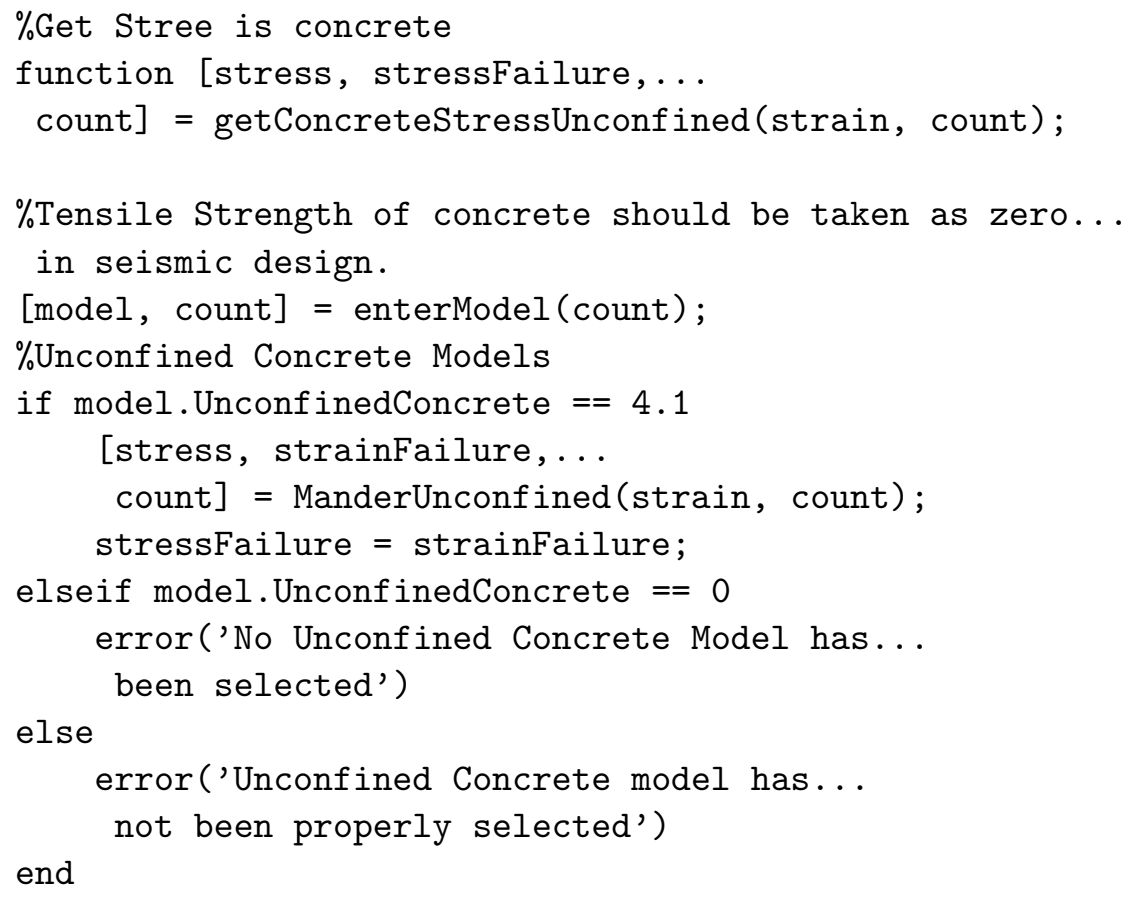

\section{B.14 Ultimate Failure Strains}

This function calculates the ultimate compressive and tensile stains the cross section can withstand.

\%Get Ultimate Compressive Strain and Ultimate... 


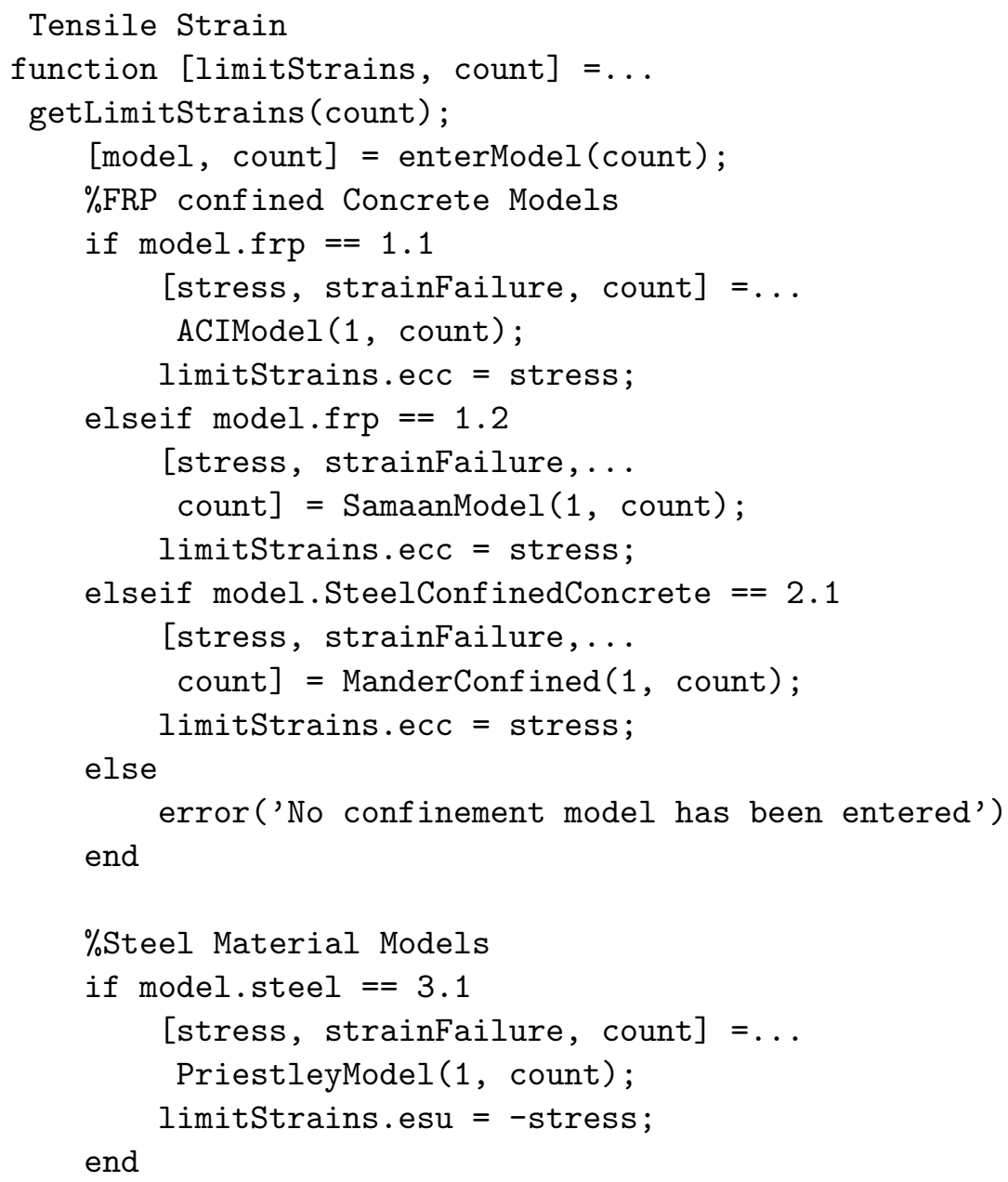

\section{B.15 Longitudinal Reinforcing Steel Area}

This function calculates the total section area of the longitudinal reinforcing steel in the cross section.

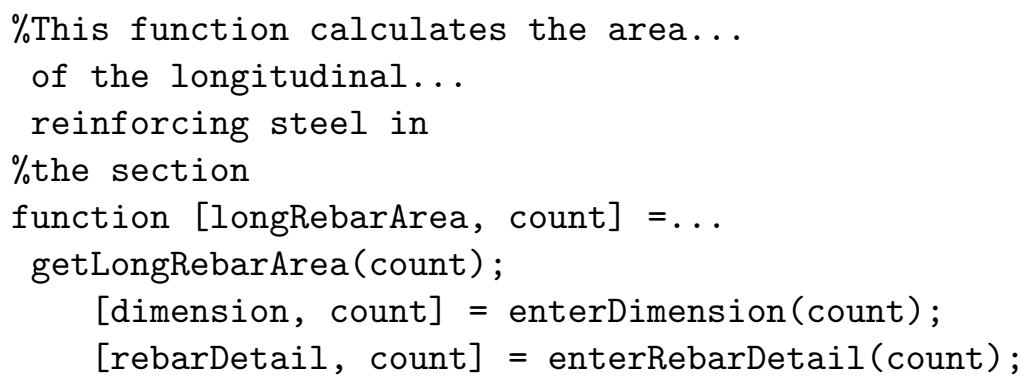




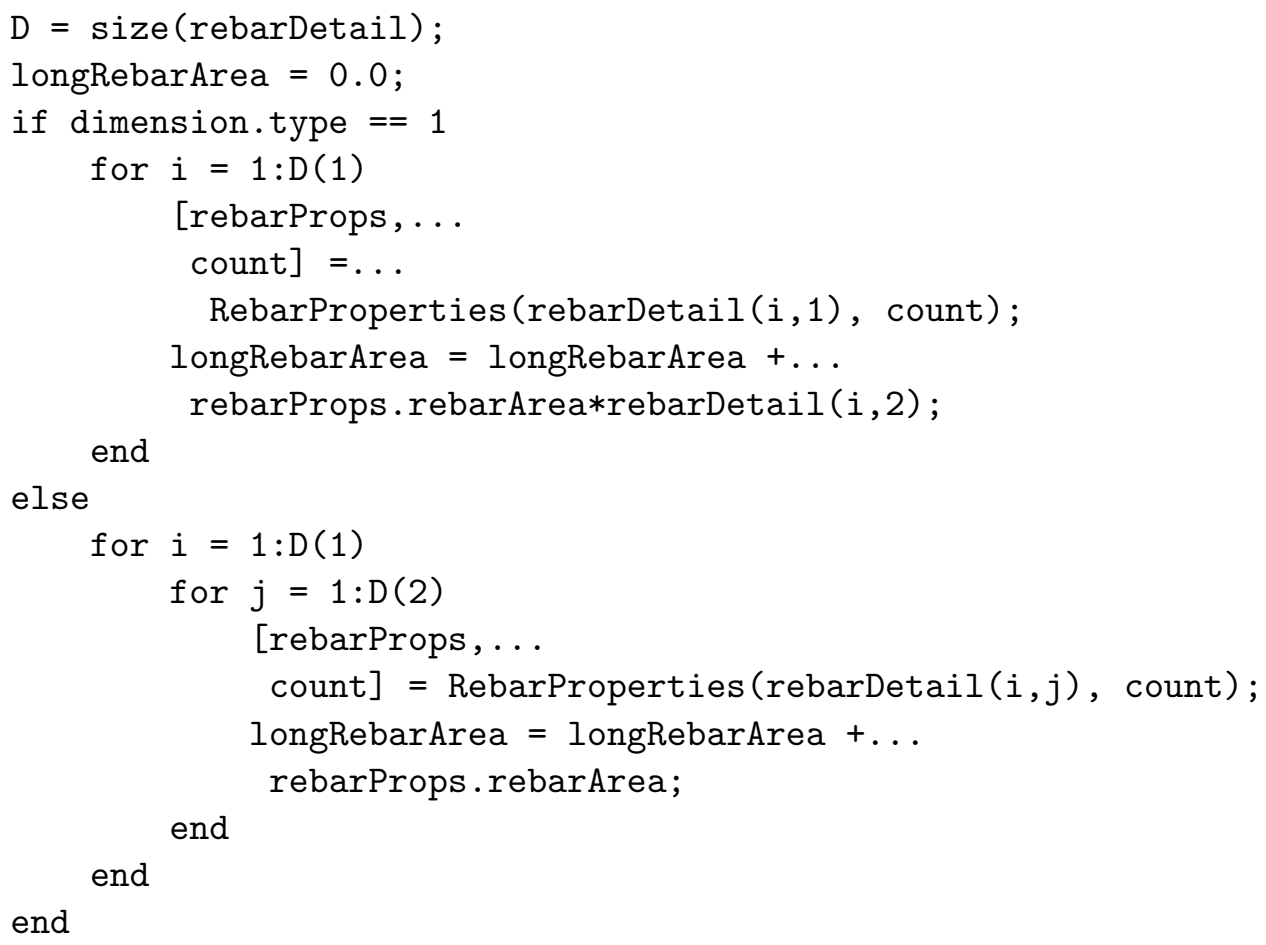

\section{B.16 Longitudinal Reinforcing Steel Ratio}

This function calculates the longitudinal reinforcing steel ratio of the cross section.

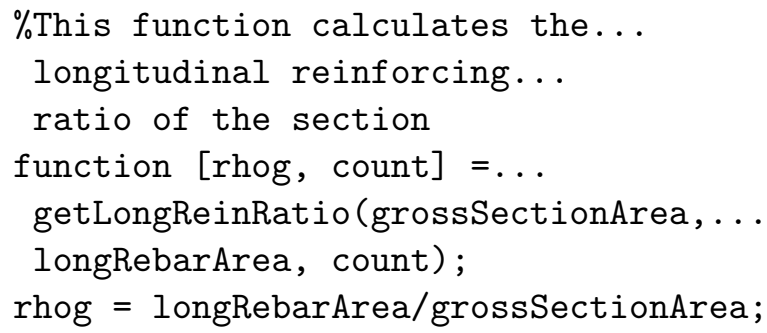

\section{B.17 Maximum Moment}

This function calculates the maximum moment the cross section can withstand.

function [maxMoment] = getMaxMoment (momentCurvature)

$[\mathrm{A}, \mathrm{B}]=\operatorname{size}($ momentCurvature);

for $i=1: A$ 


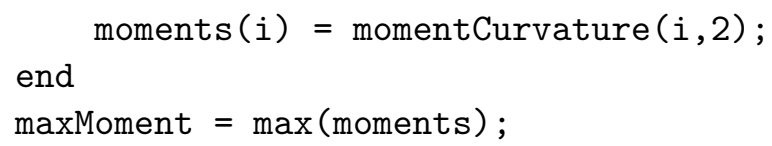

\section{B.18 Reaction Moment}

This function calculates the reaction moment induced by an applied curvature on the cross section.

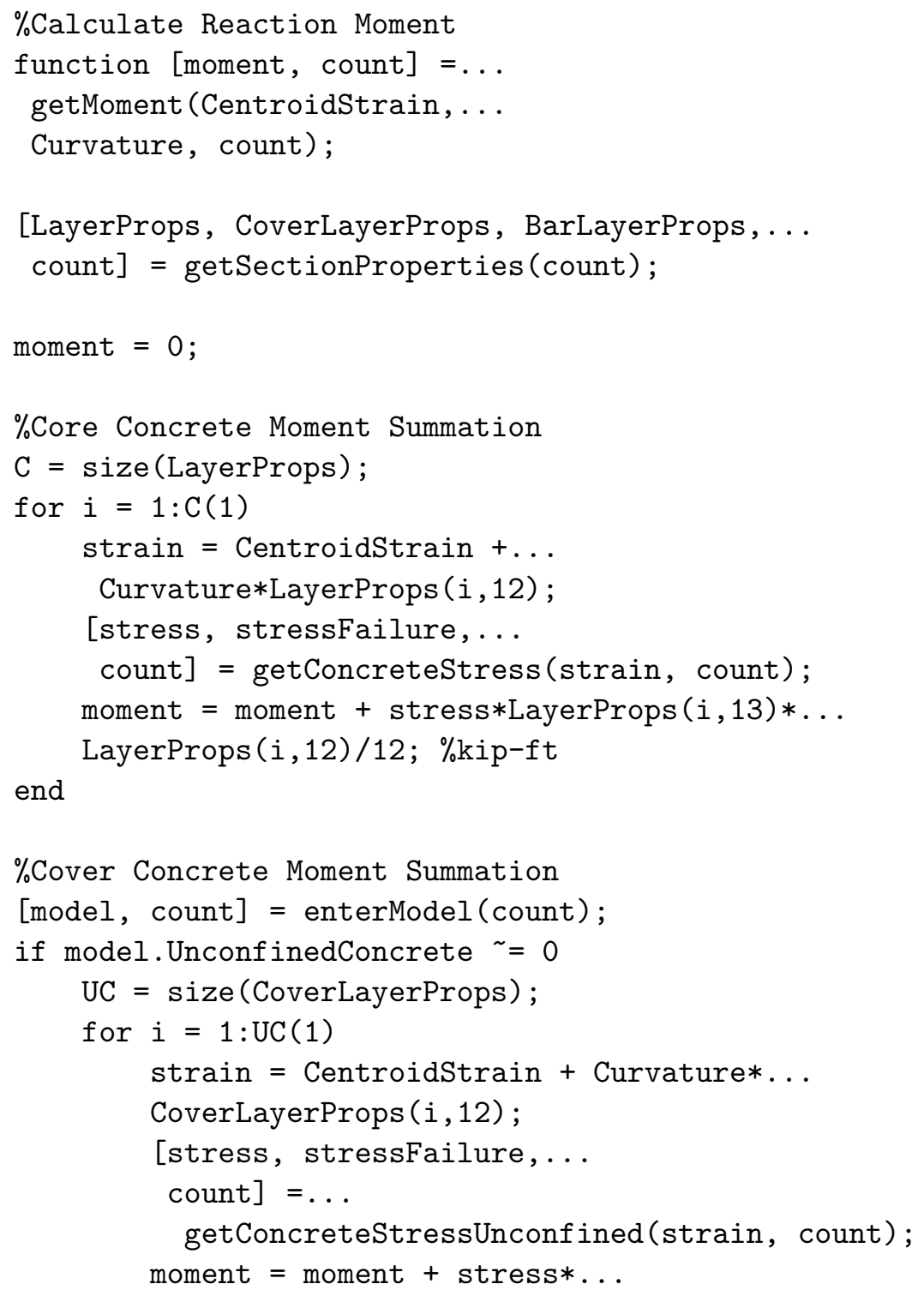


CoverLayerProps (i, 13)*CoverLayerProps (i , . .

12) $/ 12 ; \%$ kip-ft

end

end

$\%$ Steel Reaction Force Summation

$\mathrm{S}=\operatorname{size}$ (BarLayerProps);

for $i=1: S(1)$

strain = CentroidStrain + Curvature*BarLayerProps (i,13);

[stress, stressFailure,...

count $]=$ getSteelStress (strain, count);

moment $=$ moment + stress $*$ BarLayerProps $(i, 14) * \ldots$

BarLayerProps (i,13)/12; \%kip-ft

end

\section{B.19 Moment-Curvature Analysis}

This function conducts the Moment-Curvature Analysis.

$\%$ Run Moment Curvature Analysis

function [momentCurvature, count] $=\ldots$

getMomentCurvature (count);

[limitStrains, count] = getLimitStrains (count);

[LayerProps, CoverLayerProps, BarLayerProps,...

count $]=$ getSectionProperties (count);

\%Calculate the Ultimate Curvature at blalanced loading

[UltimateCurvature, count] $=\ldots$

getUltimateCurvature (count);

\%Compile Moment Curvature Pairs for loading

numPoints $=30$;

stepSize = UltimateCurvature/numPoints;

Curvature $=0$;

Point $=1$;

Failed $=0$;

ec $=$ (limitStrains.ecc + limitStrains.esu) $/ 2$;

while Failed $==0$

$\%$ Calculate the centroid strain for the given... curvature and loading 


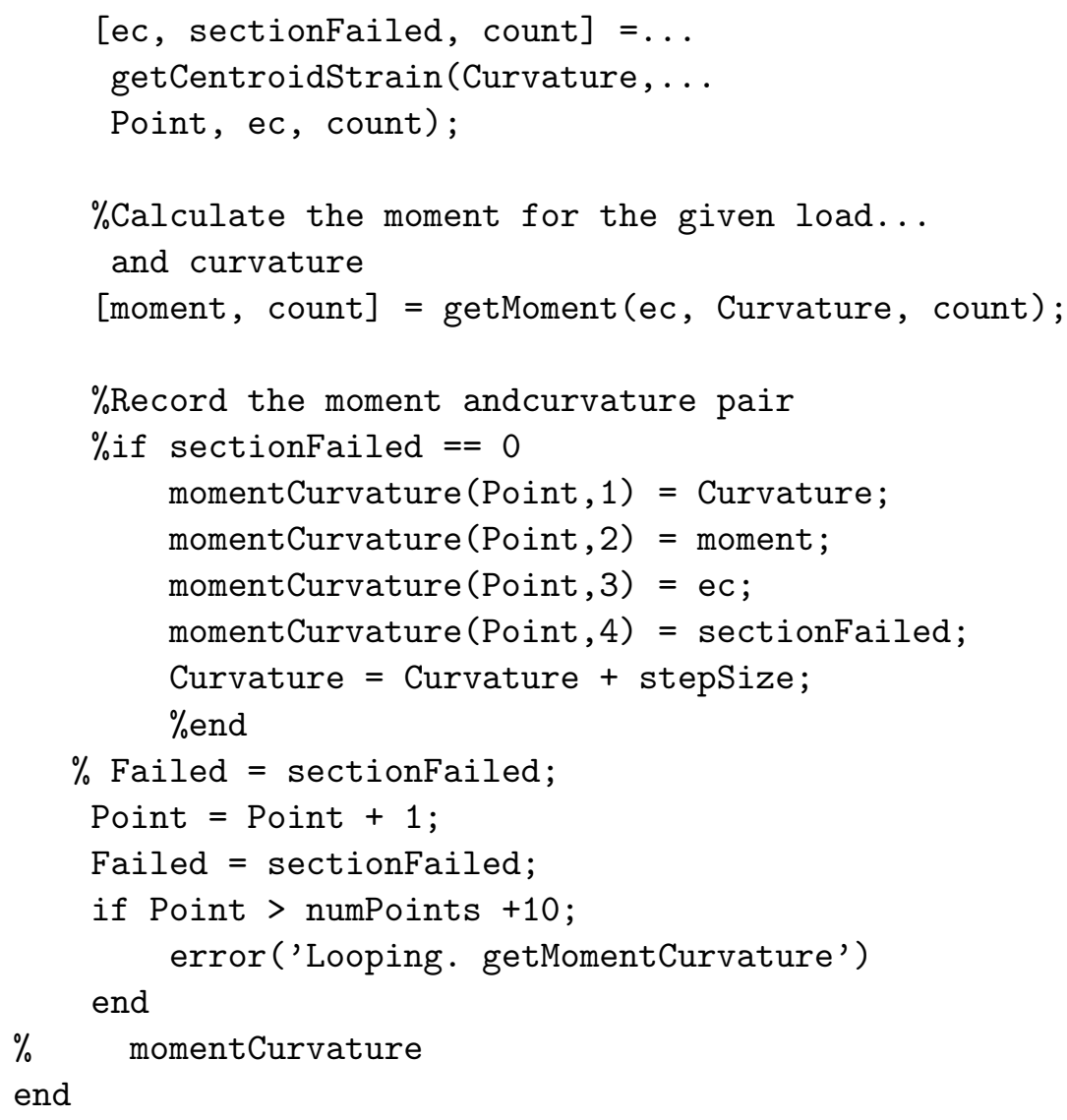

\section{B.20 MC Calculations for PM Interaction Analysis}

This function calculates limit state Moment-Curvature points for the PM Interaction Analyses.

\%Run Moment Curvature Analysis

function [momentCurvature,...

count $]=$ getMomentCurvature2 (count, load);

[limitStrains, count] = getLimitStrains (count);

[LayerProps, CoverLayerProps, BarLayerProps,...

count $]=$ getSectionProperties (count);

$\%$ Calculate the Ultimate Curvature at...

blalanced loading

[UltimateCurvature, count] $=\ldots$ 


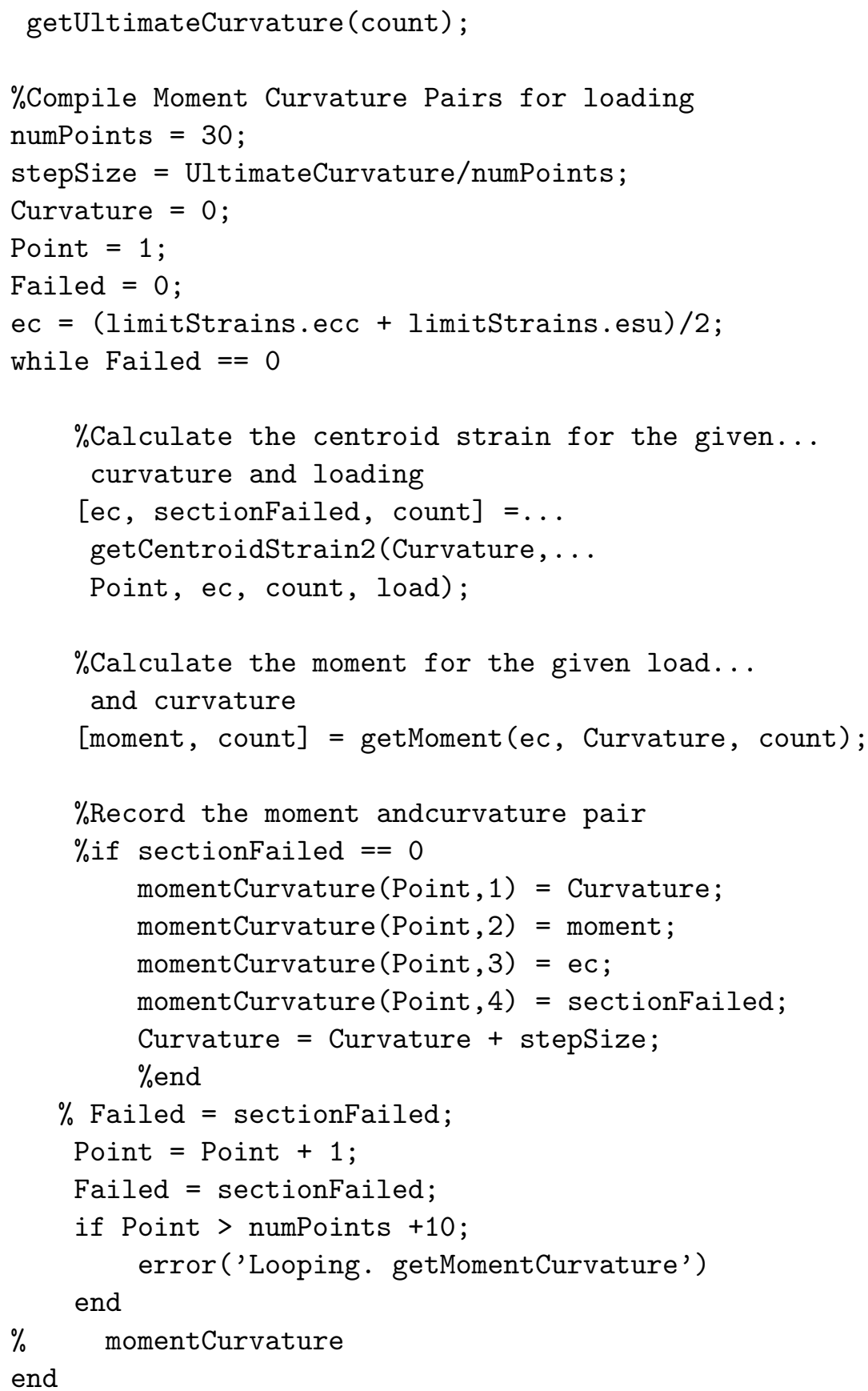




\section{B.21 Reaction Force}

This function calculates the reaction force in a cross section induced by an applied curvature.

$\%$ Calculate Reactant Axial Force

function [ReactionForce, sectionFailure,... count $]=$ getReactionForce (CentroidStrain, $\ldots$

Curvature, count);

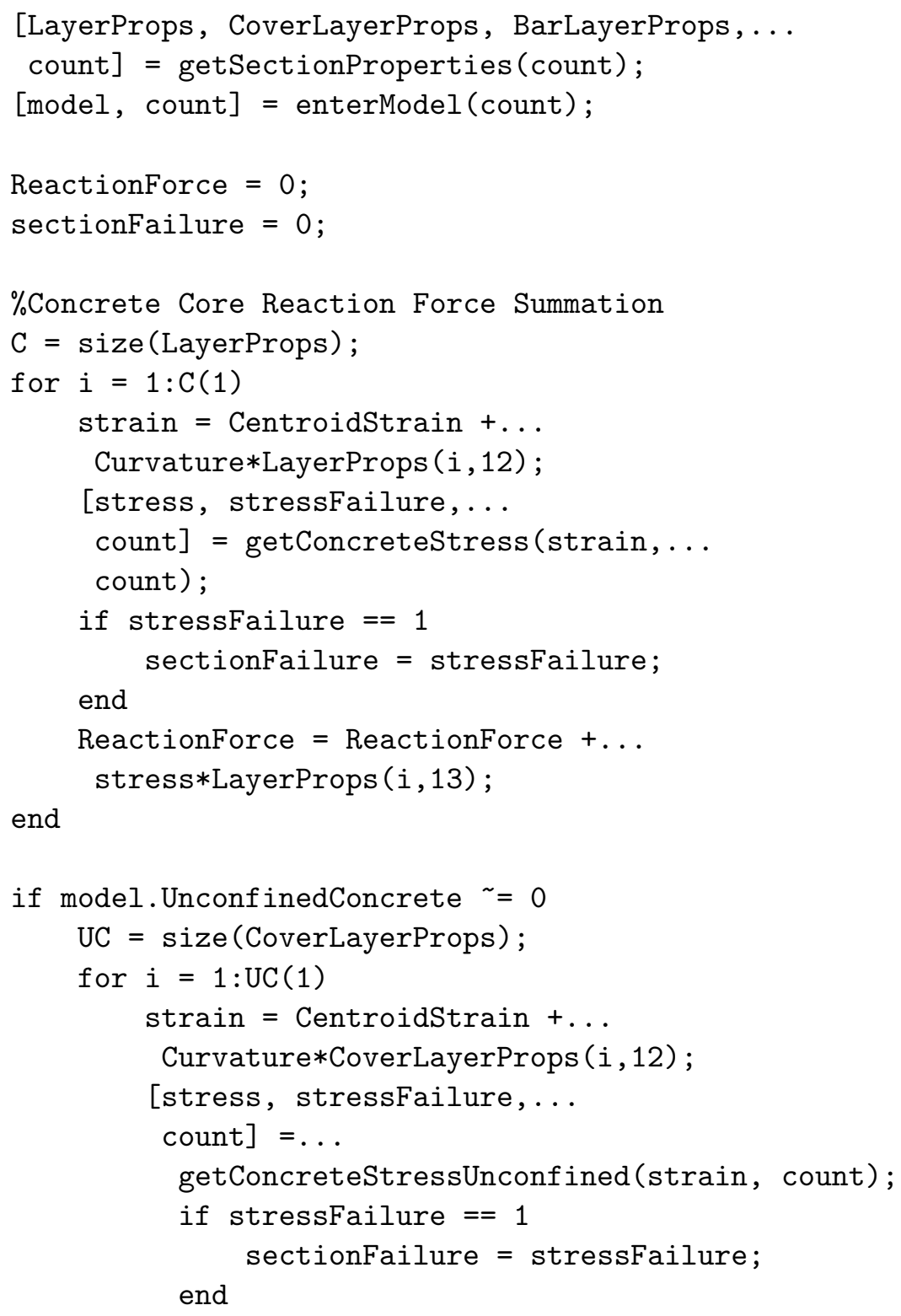




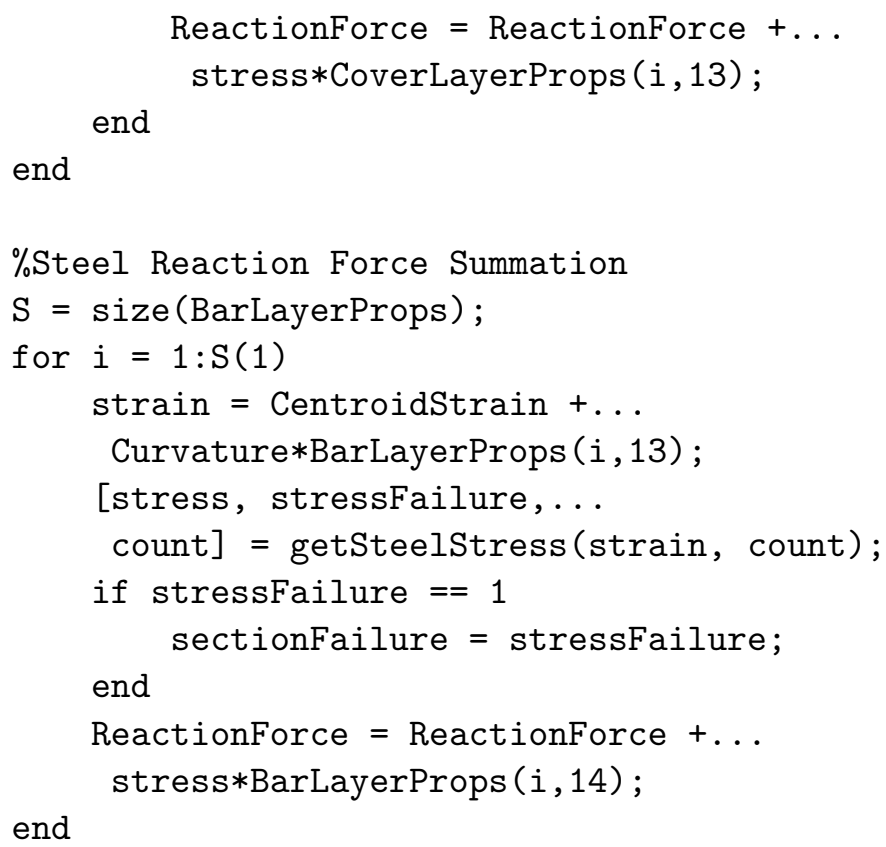

\section{B.22 Gross Section Area}

This function calculates the gross area of the cross section.

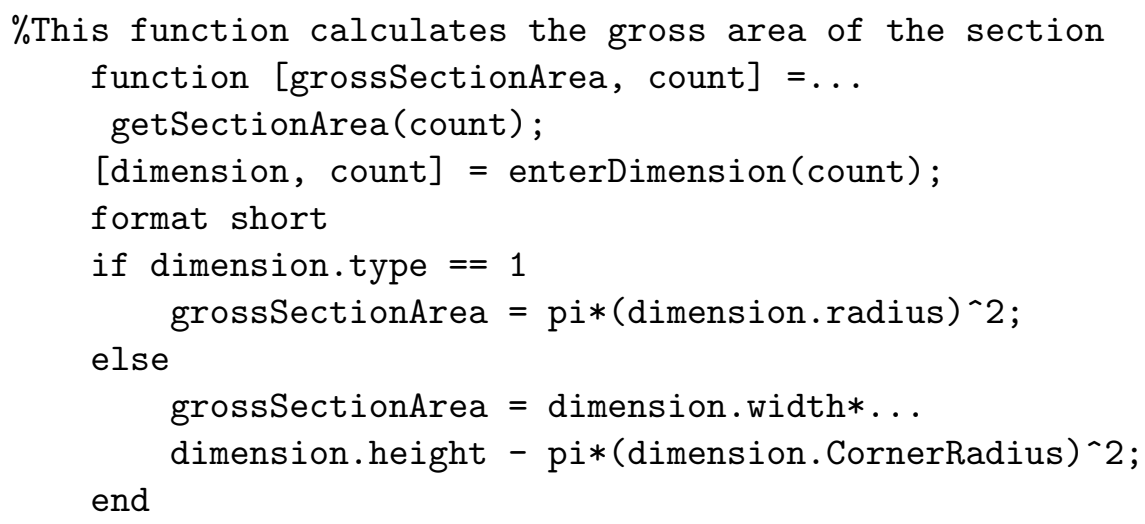

\section{B.23 Cross Section Properties}

This function maps and tags the cross section dimensions and material properties. $\%$ Maps and plots the cross section 


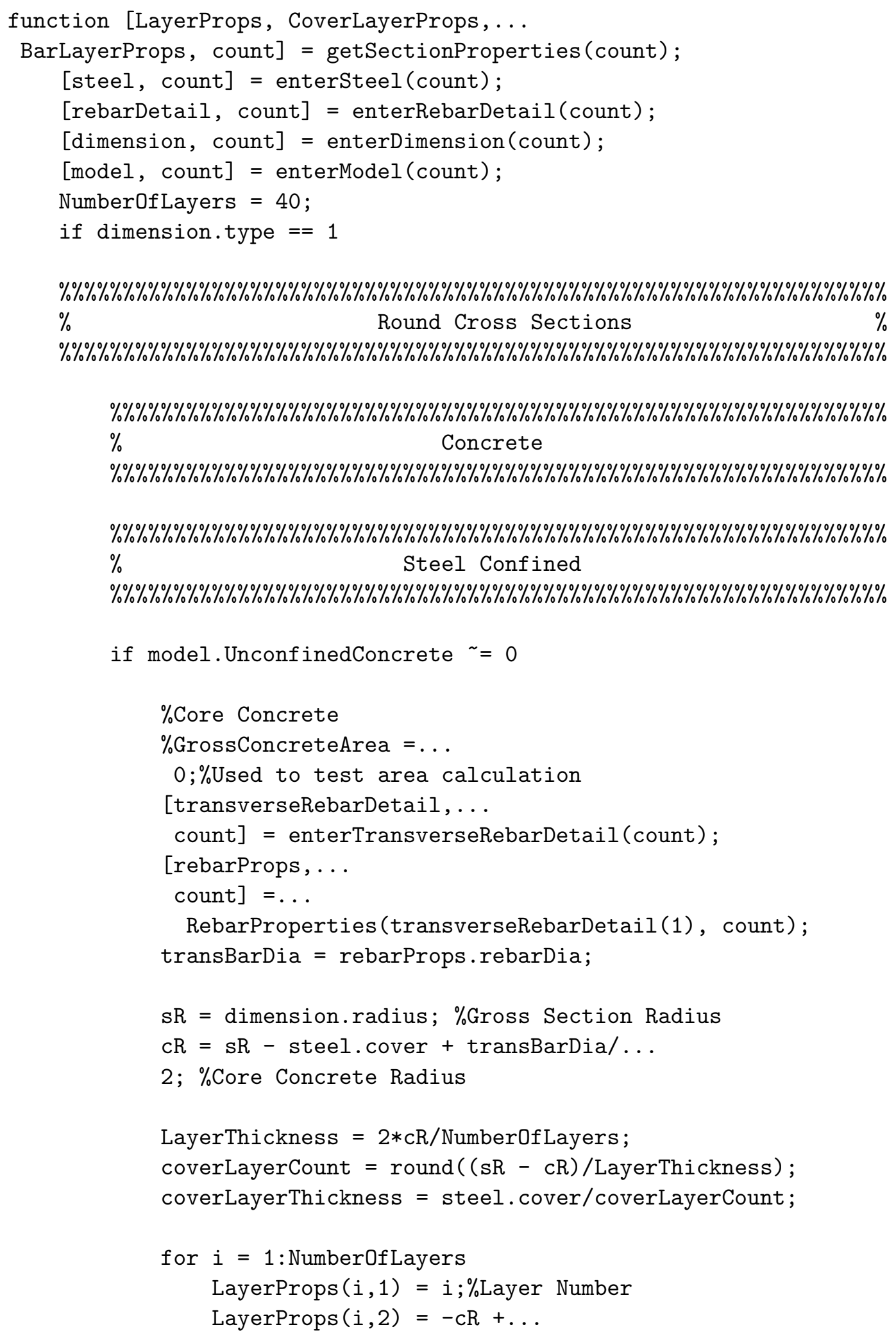


LayerThickness*( $i$ - 1);\%Bottom Right Y

$\%$ LayerProps $(i, 3)=c R * \cos (\operatorname{asin}($ LayerProps $(i, 2) / \ldots$

$\%(\mathrm{C}))$; \%Trapizoid approx has more error...

but is conservative

LayerProps $(i, 3)=c R * \cos \ldots$

(asin ( LayerProps $(i, 2)+\ldots$

LayerThickness/2)/cR)) ; \%ottom Right X

LayerProps $(i, 4)=$ LayerProps $(i, 2)+\ldots$

LayerThickness;\%Top Right Y

$\%$ LayerProps $(i, 5)=c R * \cos ($ asin (LayerProps $(i, 4) / \ldots$

$\%$ CR)) ; \% Trapizoid approx has more error...

but is conservative

LayerProps $(i, 5)=$ LayerProps $(i, 3) ; \%$ Top Right $X$

LayerProps $(i, 6)=$ LayerProps $(i, 4) ; \%$ Top Left $Y$

LayerProps $(i, 7)=$-LayerProps $(i, 5) ; \%$ Top Left $X$

LayerProps $(i, 8)=$ LayerProps $(i, 2) ; \%$ Bottom Left $Y$

LayerProps $(i, 9)=-$ LayerProps $(i, \ldots$

3) ;\%Bottom Left $X$

LayerProps $(i, 10)=$ LayerProps $(i, \ldots$

2);\%Bottom Right $Y$

LayerProps $(i, 11)=$ LayerProps $(i, \ldots$

3) ;\%Bottom Right $X$

LayerProps $(i, 12)=$ LayerProps $(i, 2)+\ldots$

LayerThickness $/ 2 ; \%$ Vertical Centerline

LayerProps $(i, 13)=($ LayerProps $(i, 4)-\ldots$

LayerProps $(i, 2)) * \ldots$

(LayerProps (i,3) - LayerProps (i,9));\%Area

LayerProps $(i, 14)=$ LayerThickness;\%Layer Thickness

$\%$ GrossConcreteArea $=$ GrossConcreteArea $+\ldots$

LayerProps (i,13);\%Used to test area calculation

end

$\%$ Cover Concrete

$\%$ Bottom

for $i=1$ : coverLayerCount

CoverLayerProps $(i, 1)=i ; \%$ Layer Number

CoverLayerProps $(i, 2)=-s R+\ldots$

coverLayerThickness*( $i$ - 1); \%ottom Right Y

CoverLayerProps $(i, 3)=\operatorname{sR} * \cos (\operatorname{asin} .$.

( CoverLayerProps $(i, 2)+\ldots$

coverLayerThickness/2)/sR)); \%Bottom Right X

CoverLayerProps $(i, 4)=$ CoverLayerProps $(i, 2)+\ldots$ coverLayerThickness;\%Top Right Y 


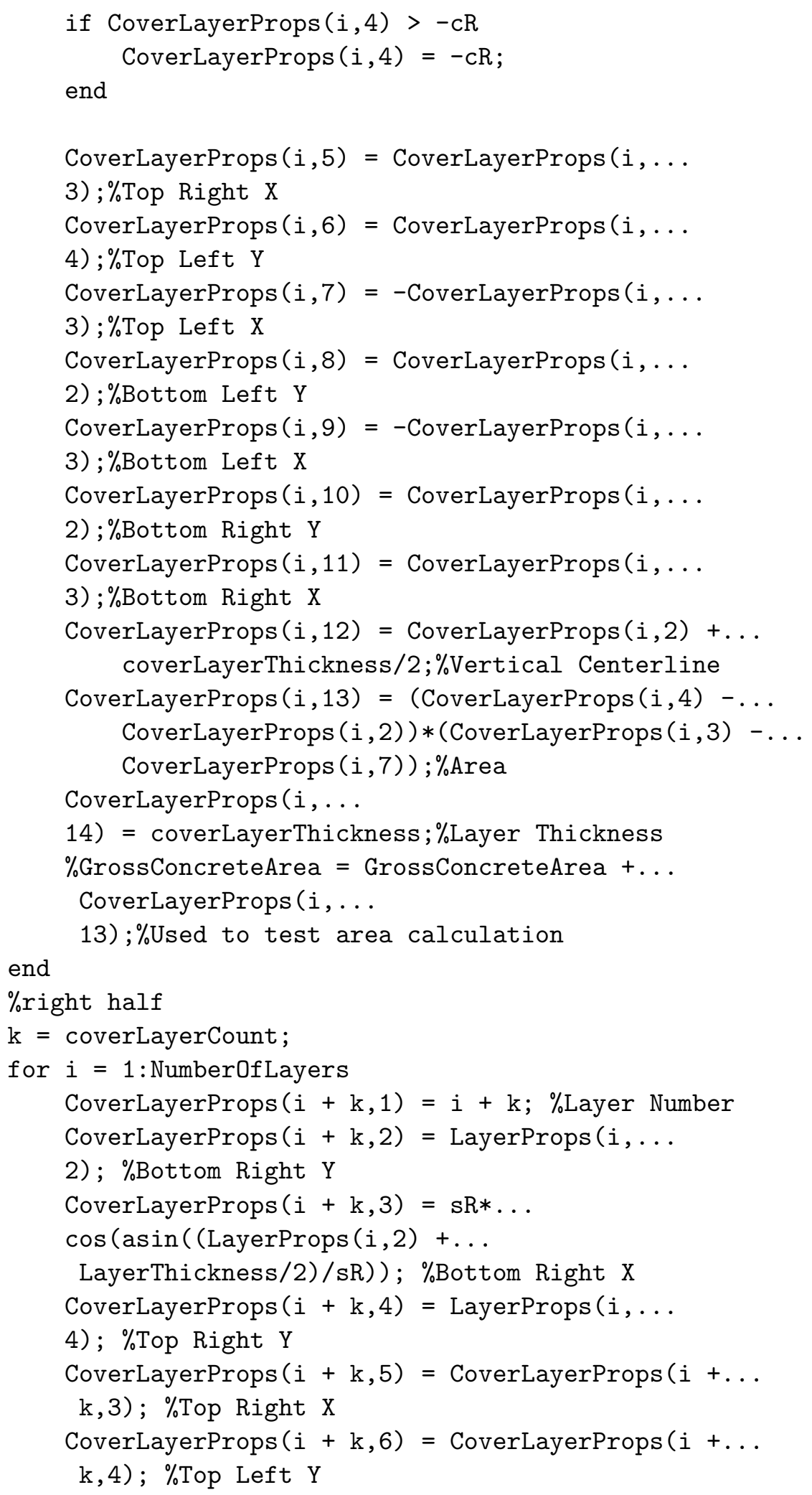


CoverLayerProps $(i+k, 7)=\operatorname{LayerProps}(i, \ldots$

5) ; \%Top Left $X$

CoverLayerProps $(i+k, 8)=$ CoverLayerProps $(i+\ldots$

$\mathrm{k}, 2)$; \%ottom Left $\mathrm{Y}$

CoverLayerProps $(i+k, 9)=$ CoverLayerProps $(i+\ldots$

$\mathrm{k}, 7)$; \%ottom Left $\mathrm{X}$

CoverLayerProps $(i+k, 10)=$ CoverLayerProps $(i+\ldots$

$\mathrm{k}, 2)$; \%ottom Right $\mathrm{Y}$

CoverLayerProps $(i+k, 11)=$ CoverLayerProps $(i+\ldots$

$\mathrm{k}, 3)$; \%ottom Right $\mathrm{X}$

CoverLayerProps $(i+k, \ldots$

12) $=$ CoverLayerProps $(i+k, 2)+\ldots$

LayerThickness/2; \%Vertical Centerline

CoverLayerProps $(i+k, 13)=($ CoverLayerProps $(i+\ldots$

$\mathrm{k}, 3)$ - CoverLayerProps $(i+\mathrm{k}, 7)) * \ldots$

(CoverLayerProps $(i+k, 4)-\ldots$

CoverLayerProps $(i+\mathrm{k}, 2))$; \% Area

CoverLayerProps $(i+k, 14)=$ LayerThickness; \%Layer...

Thickness

$\%$ GrossConcreteArea $=$ GrossConcreteArea $+\ldots$

CoverLayerProps $(i+\ldots$

$\mathrm{k}, 13)$; $\%$ Used to test area calculation

end

$\%$ Top

$\mathrm{k}=\mathrm{k}+$ NumberOfLayers;

for $i=1$ : coverLayerCount

CoverLayerProps $(i+k, 1)=i+k ; \%$ Layer Number

CoverLayerProps $(i+k, 2)=c R+\ldots$

coverLayerThickness* $(\mathrm{i}-\ldots$

1) ;\%Bottom Right $Y$

CoverLayerProps $(i+k, 3)=s R * \ldots$

$\cos$ (asin ( CoverLayerProps $(i+k, 2)+\ldots$

coverLayerThickness/2)/sR));\%Bottom Right X

CoverLayerProps $(i+k, 4)=$ CoverLayerProps $(i+\ldots$ $\mathrm{k}, 2)+$ coverLayerThickness;\%Top Right $\mathrm{Y}$

if CoverLayerProps $(i+\mathrm{k}, 4)>\mathrm{sR}$

CoverLayerProps $(i+k, 4)=s R$;

end

CoverLayerProps $(i+k, 5)=$ CoverLayerProps $(i+\ldots$ $\mathrm{k}, 3)$; Top Right $\mathrm{X}$

CoverLayerProps $(i+k, 6)=\operatorname{CoverLayerProps}(i+\ldots$ $\mathrm{k}, 4) ; \%$ Top Left $\mathrm{Y}$ 
CoverLayerProps $(i+k, 7)=-$ CoverLayerProps $(i+\ldots$ $k, 3) ; \%$ Top Left $X$

CoverLayerProps $(i+k, 8)=$ CoverLayerProps $(i+\ldots$ $k, 2) ; \%$ Bottom Left $Y$

CoverLayerProps $(i+k, 9)=$ CoverLayerProps $(i+\ldots$ $\mathrm{k}, 7) ; \%$ Bottom Left $\mathrm{X}$

CoverLayerProps $(i+k, 10)=$ CoverLayerProps $(i+\ldots$ $\mathrm{k}, 2) ; \%$ Bottom Right $\mathrm{Y}$

CoverLayerProps $(i+k, 11)=$ CoverLayerProps $(i+\ldots$ $\mathrm{k}, 3) ; \%$ Bottom Right $\mathrm{X}$

CoverLayerProps $(i+k, 12)=$ CoverLayerProps $(i+\ldots$ $k, 2)+$ coverLayerThickness $/ 2 ; \%$ Vertical Centerline CoverLayerProps $(i+k, 13)=$ (CoverLayerProps $(i+\ldots$ $k, 4)$ - CoverLayerProps $(i+\ldots$

$\mathrm{k}, 2)) *($ CoverLayerProps $(i+\mathrm{k}, 3)-\ldots$ CoverLayerProps $(i+k, 7)) ; \%$ Area

CoverLayerProps $(i+\ldots$

$\mathrm{k}, 14)=$ coverLayerThickness $; \%$ Layer Thickness

$\%$ GrossConcreteArea $=$ GrossConcreteArea $+\ldots$

CoverLayerProps $(i+\ldots$

$k, 13) ; \%$ Used to test area calculation

end

$\%$ left half

$\mathrm{k}=\mathrm{k}+$ coverLayerCount;

for $i=1$ :NumberOfLayers

CoverLayerProps $(i+k, 1)=i+k ; \%$ Layer Number

CoverLayerProps $(i+k, 2)=$ CoverLayerProps $(1-i+\ldots$ coverLayerCount + NumberOfLayers, 2);\%Bottom Right Y CoverLayerProps $(i+k, 3)=-$ CoverLayerProps $(1-i+\ldots$ coverLayerCount + NumberOfLayers, 9);\%Bottom Right X CoverLayerProps $(i+k, 4)=$ CoverLayerProps $(1-i+\ldots$ coverLayerCount + NumberOfLayers, 4$) ; \%$ Top Right $Y$ CoverLayerProps $(i+k, 5)=-$ CoverLayerProps $(1-i+\ldots$ coverLayerCount + NumberOfLayers, 7$) ; \%$ Top Right X CoverLayerProps $(i+k, 6)=$ CoverLayerProps $(1-i+\ldots$ coverLayerCount + NumberOfLayers, 6$) ; \%$ Top Left Y

CoverLayerProps $(i+k, 7)=-$ CoverLayerProps $(1-i+\ldots$ coverLayerCount + NumberOfLayers, 5$) ; \%$ Top Left X CoverLayerProps $(i+k, 8)=$ CoverLayerProps $(1-i+\ldots$ coverLayerCount + NumberOfLayers, 8$) ; \%$ Bottom Left $\mathrm{Y}$ CoverLayerProps $(i+k, 9)=-$ CoverLayerProps $(1-i+\ldots$ coverLayerCount + NumberOfLayers,11);\%Bottom Left X CoverLayerProps $(i+k, 10)=$ CoverLayerProps $(1-i+\ldots$ coverLayerCount + NumberOfLayers,10);\%Bottom Right Y 
CoverLayerProps $(i+k, 11)=-$ CoverLayerProps $(1-i+\ldots$ coverLayerCount + NumberOfLayers, 9$) ; \%$ Bottom Right X CoverLayerProps $(i+k, 12)=$ CoverLayerProps $(1-i+\ldots$ coverLayerCount + NumberOfLayers, ...

12);\%Vertical Centerline

CoverLayerProps $(i+k, 13)=$ CoverLayerProps $(1-i+\ldots$ coverLayerCount + NumberOfLayers, 13);\%Area

CoverLayerProps $(i+k, \ldots$

14) = LayerThickness;\%Layer Thickness

$\%$ GrossConcreteArea $=$ GrossConcreteArea $+\ldots$

CoverLayerProps ( $\mathrm{i}+\mathrm{k}, \ldots$

13) ; \% Used to test area calculation

end

CoverLayerProps

coverLayerCount

pause

else

$\% \% \% \% \% \% \% \% \% \% \% \% \% \% \% \% \% \% \% \% \% \% \% \% \% \% \% \% \% \% \% \% \% \% \% \% \% \% \% \% \% \% \% \% \% \% \% \% \% \% \% \% \% \%$

$\% \quad$ FRP Confined

$\% \% \% \% \% \% \% \% \% \% \% \% \% \% \% \% \% \% \% \% \% \% \% \% \% \% \% \% \% \% \% \% \% \% \% \% \% \% \% \% \% \% \% \% \% \% \% \% \% \% \% \% \% \% \% \% \% \%$

CoverLayerProps $=0$;

LayerThickness $=2 *$ dimension.radius/NumberOfLayers;

$\%$ GrossConcreteArea $=0 ; \%$ Used to test area calculation

for $i=1$ :NumberOfLayers

LayerProps $(i, 1)=i ; \%$ Layer Number

LayerProps $(i, 2)=-$ dimension.radius $+\ldots$

LayerThickness*(i - 1);\%Bottom Right Y

$\%$ LayerProps $(i, 3)=$ dimension.radius*...

cos (asin (LayerProps $(i, 2) / \ldots$

\%dimension.radius)); \%Trapizoid approx has...

more error but is conservative

LayerProps $(i, 3)=$ dimension.radius $* \cos \ldots$

(asin ( LayerProps $(i, 2)+\ldots$

LayerThickness/2)/...

dimension.radius)); \%Bottom Right $\mathrm{X}$

LayerProps $(i, 4)=$ LayerProps $(i, 2)+\ldots$

LayerThickness;\%Top Right Y

$\%$ LayerProps $(i, 5)=$ dimension.radius $* \cos \ldots$

(asin (LayerProps $(i, 4) / \ldots$

$\%$ dimension.radius)) ; \% Trapizoid approx has more... error but is conservative

LayerProps $(i, 5)=$ LayerProps (i,3);\%Top Right X 
LayerProps $(i, 6)=$ LayerProps $(i, 4) ; \%$ Top Left $Y$

LayerProps $(i, 7)=$-LayerProps $(i, 5) ; \%$ Top Left $X$

LayerProps $(i, 8)=$ LayerProps $(i, 2) ; \%$ Bottom Left $Y$

LayerProps $(i, 9)=-$ LayerProps $(i, 3) ; \%$ Bottom Left $X$

LayerProps $(i, 10)=$ LayerProps $(i, 2) ; \%$ Bottom Right $Y$

LayerProps $(i, 11)=$ LayerProps $(i, 3) ; \%$ Bottom Right $X$

LayerProps $(i, 12)=$ LayerProps $(i, 2)+\ldots$

LayerThickness $/ 2 ; \%$ Vertical Centerline

LayerProps $(i, 13)=$ (LayerProps $(i, 4)-$ LayerProps $(i, 2)) * \ldots$

(LayerProps (i,3) - LayerProps (i,9));\%Area

LayerProps $(i, 14)=$ LayerThickness $\%$ Layer Thickness

$\%$ GrossConcreteArea $=$ GrossConcreteArea $+\ldots$

LayerProps $(i, 13) ; \%$ Used to test area calculation

end

end

\% \% \% \% \% \% \% \% \% \% \% \% \% \% \% \% \% \% \% \% \% \% \% \% \% \% \% \% \% \% \% \% \% \% \% \% \% \% \% \% \% \% \% \% \% \% \% \% \% \% \% \% \% \% \% \% \% \% \% \% \% \% \% \% \% \%

$\% \quad$ Rebar

\% \% \% \% \% \% \% \% \% \% \% \% \% \% \% \% \% \% \% \% \% \% \% \% \% \% \% \% \% \% \% \% \% \% \% \% \% \% \% \% \% \% \% \% \% \% \% \% \% \% \% \% \% \% \% \% \% \% \% \% \% \% \% \% \% \% \% \% \% \% \% \% \%

$[\mathrm{a}, \mathrm{b}]=\operatorname{size}($ rebarDetail $)$;

perimeterBarCount $=0$;

TotalRebarArea $=0 ; \%$ Used only to test the areas

for $i=1: a$

perimeterBarCount $=$ perimeterBarCount $+\operatorname{rebarDetail}(i, 2)$;

end

AngularSpacing $=2 *$ pi/perimeterBarCount;

$\mathrm{k}=0$;

$\mathrm{z}=0$;

for $i=1$ :perimeterBarCount $\%$ First bar is at 0 degrees...

and is counted up ccw

$\mathrm{k}=\mathrm{i}-\mathrm{a} * \mathrm{z}$

if $\mathrm{k}==\mathrm{a}$

$z=z+1$

end

[rebarProps,...

count $]=$ Rebarproperties (rebarDetail $(\mathrm{k}, 1)$, count);

rebarDias $(i)=$ rebarProps.rebarDia;

barCentersX $(i)=$ (dimension.radius $-\ldots$

steel.cover - rebarDias $(i) / 2) * \ldots$

$\cos ((i-1) *$ AngularSpacing);

$\operatorname{barCentersY}(i)=$ (dimension.radius $-\ldots$

steel.cover - rebarDias $(i) / 2) * \ldots$

$\sin ((i-1) *$ AngularSpacing);

$\mathrm{m}=\operatorname{round}($ Number0fLayers $/ 3)$; 


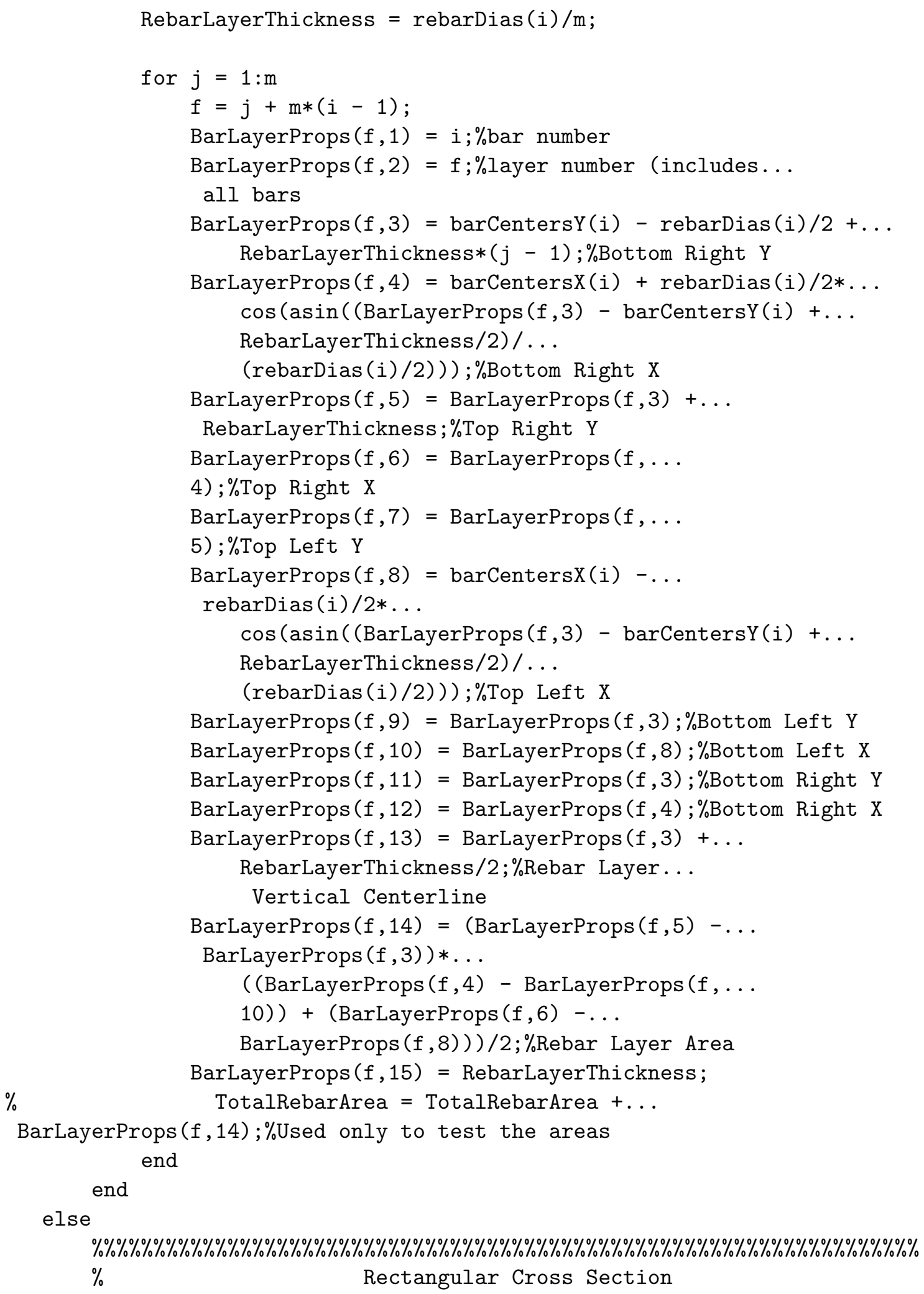


\%\%\%\%\%\%\%\%\%\%\%\%\%\%\%\%\%\%\%\%\%\%\%\%\%\%\%\%\%\%\%\%\%\%\%\%\%\%\%\%\%\%\%\%\%\%\%\%\%\%\%\%\%\%\%\%\%\%\%\%\%\%\%\%\%\%\%\%\%\%\%\%

\% \% \% \% \% \% \% \% \% \% \% \% \% \% \% \% \% \% \% \% \% \% \% \% \% \% \% \% \% \% \% \% \% \% \% \% \% \% \% \% \% \% \% \% \% \% \% \% \% \% \% \% \% \% \% \% \% \% \% \% \% \% \% \% \% \% \% \% \% \% \% \% \% \%

$\%$

Concrete

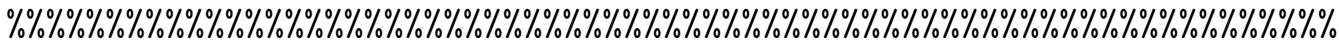

LayerThickness = dimension.height/NumberOfLayers;

$\mathrm{y}=0$;

$\%$

GrossConcreteArea $=0 ; \%$ used only to test the areas

for $i=1:$ NumberOfLayers

LayerProps $(i, 1)=i ; \%$ Layer Number

LayerProps $(i, 2)=-$ dimension.height $/ 2+\ldots$

LayerThickness*(i - 1);\%Bottom Right Y

if LayerProps $(i, 2)<-$ dimension.height $/ 2+\ldots$

dimension.CornerRadius + LayerThickness $/ 2$

LayerProps $(i, 3)=$ dimension. width $/ 2-\ldots$

dimension. CornerRadius $+\ldots$.

dimension.CornerRadius*...

$\cos$ (asin ( (-dimension. CornerRadius $+\ldots$

LayerThickness*(i - 1) + LayerThickness/2)/...

dimension.CornerRadius)) ; \%Bottom Right X

elseif LayerProps $(i, 2)<$ dimension.height/2 - ..

dimension.CornerRadius - LayerThickness $/ 2$

LayerProps $(i, 3)=$ dimension.width $/ 2 ; \%$ Bottom Right $\mathrm{X}$ else

LayerProps $(i, 3)=$ dimension.width $/ 2-\ldots$

dimension.CornerRadius $+\ldots$

dimension.CornerRadius*...

$\cos ($ asin ( ( LayerThickness* (y) $+\ldots$

LayerThickness/2)/...

dimension.CornerRadius))) ; \%ottom Right X

$\mathrm{y}=\mathrm{y}+1$

end

LayerProps $(i, 4)=$ LayerProps $(i, 2)+\ldots$

LayerThickness;\%Top Right Y

LayerProps $(i, 5)=$ LayerProps $(i, 3) ; \%$ Top Right $X$

LayerProps $(i, 6)=$ LayerProps $(i, 4) ; \%$ Top Left $Y$

LayerProps $(i, 7)=-$ LayerProps $(i, 3) ; \%$ Top Left $X$

LayerProps $(i, 8)=$ LayerProps $(i, 2) ; \%$ Bottom Left $Y$

LayerProps $(i, 9)=-$ LayerProps $(i, 3) ; \%$ Bottom Left $X$

LayerProps $(i, 10)=$ LayerProps $(i, 2) ; \%$ Bottom Right $Y$

LayerProps $(i, 11)=$ LayerProps $(i, 3) ; \%$ Bottom Right $X$

LayerProps $(i, 12)=$ LayerProps $(i, 2)+\ldots$

LayerThickness/2;\%Layer Vertical Centerline 


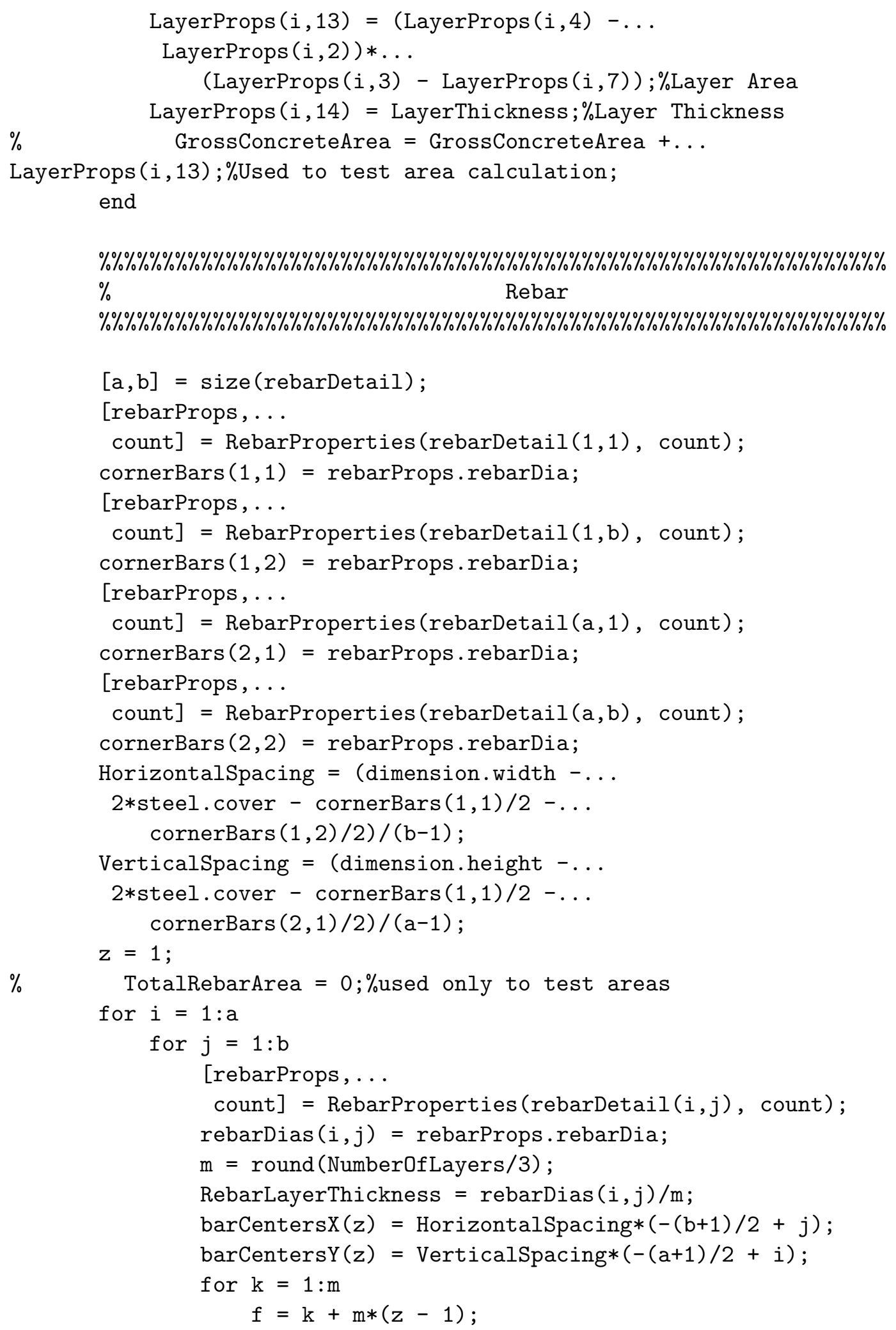




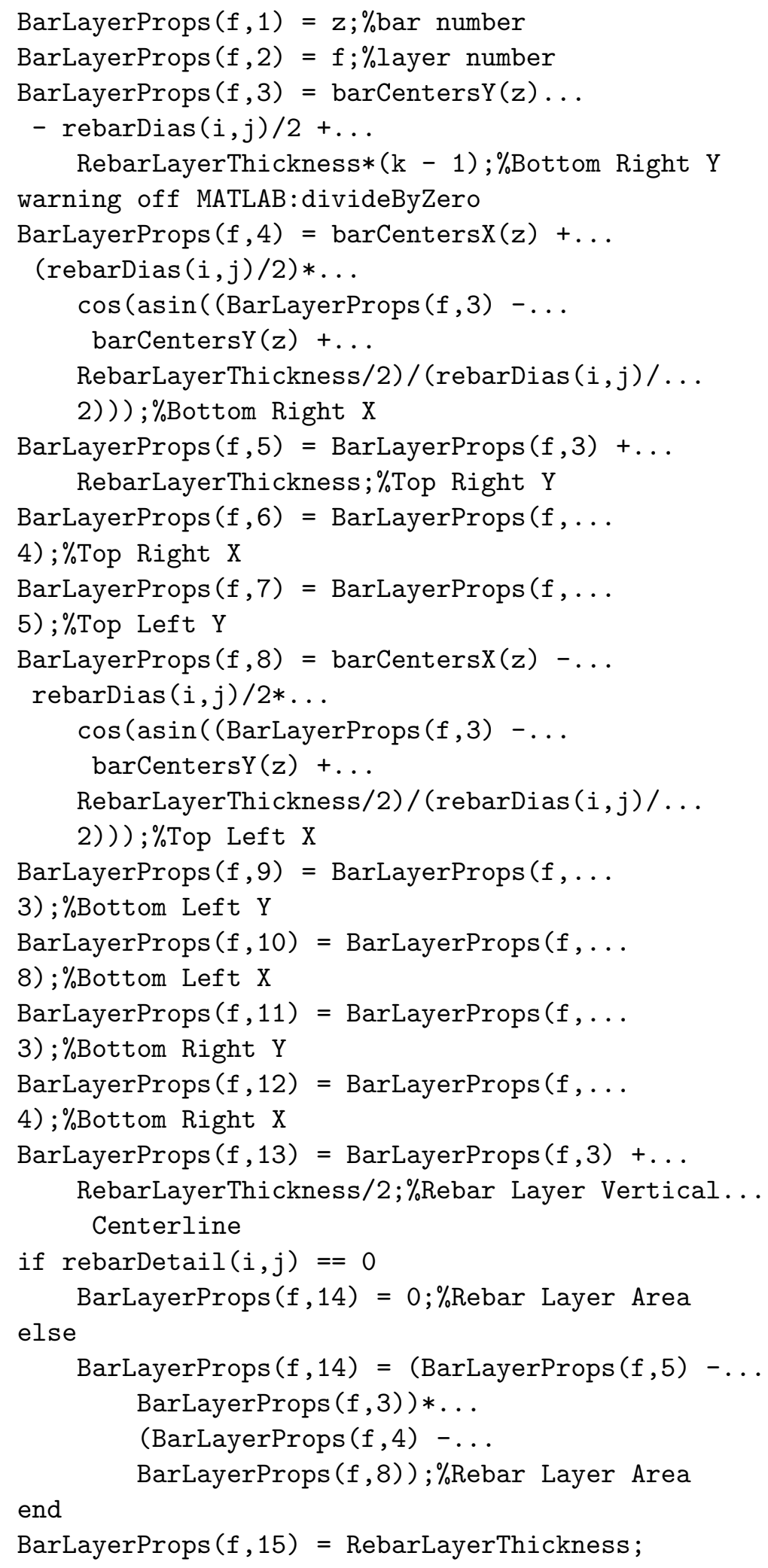


BarLayerProps (f,14);\%used only to test the areas end $z=z+1$ end end end

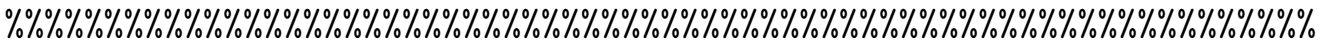
$\% \quad$ Subtract steel from concrete area \%\%\%\% \% \% \% \% \% \% \% \% \% \% \% \% \% \% \% \% \% \% \% \% \% \% \% \% \% \% \% \% \% \% \% \% \% \% \% \% \% \% \% \% \% \% \% \% \% \% \% \% \% \% \% \% \% \% \% \% \% \% \% \% \% \% \% \% \%

$[\mathrm{a}, \mathrm{b}]=\operatorname{size}($ LayerProps);

$[\mathrm{c}, \mathrm{d}]=\operatorname{size}($ BarLayerProps);

$\% \quad$ NetConcreteArea $=0 ; \%$ used only to test the areas for $i=1: a$

for $j=1: c$

if $\operatorname{BarLayerProps}(j, 13)>=$ LayerProps $(i, 12)-\ldots$

LayerThickness $/ 2$

if BarLayerProps $(j, 13)<$ LayerProps $(i, 12)+\ldots$

LayerThickness $/ 2$

LayerProps $(i, 13)=$ LayerProps $(i, 13)-\ldots$ BarLayerProps $(j, 14)$; end

end

end

$\% \quad$ NetConcreteArea $=$ NetConcreteArea $+\ldots$

LayerProps (i,13); \%used only to test areas

end

$\% \quad$ TotalRebarArea\%used only to test areas

$\% \quad$ GrossConcreteArea\%used only to test areas

$\% \quad$ NetConcreteArea\%used only to test areas

$\% \quad$ AreaError = (GrossConcreteArea - NetConcreteArea $)-\ldots$

TotalRebarArea\%used only to test areas

\section{B.24 Longitudinal Reinforcement Stress}

This function calculates the stress in longitudinal reinforcement for a given stain.

$\%$ Get Stress in longitudinal steel

function [stress, stressFailure,...

count $]=$ getSteelStress (strain, count); 


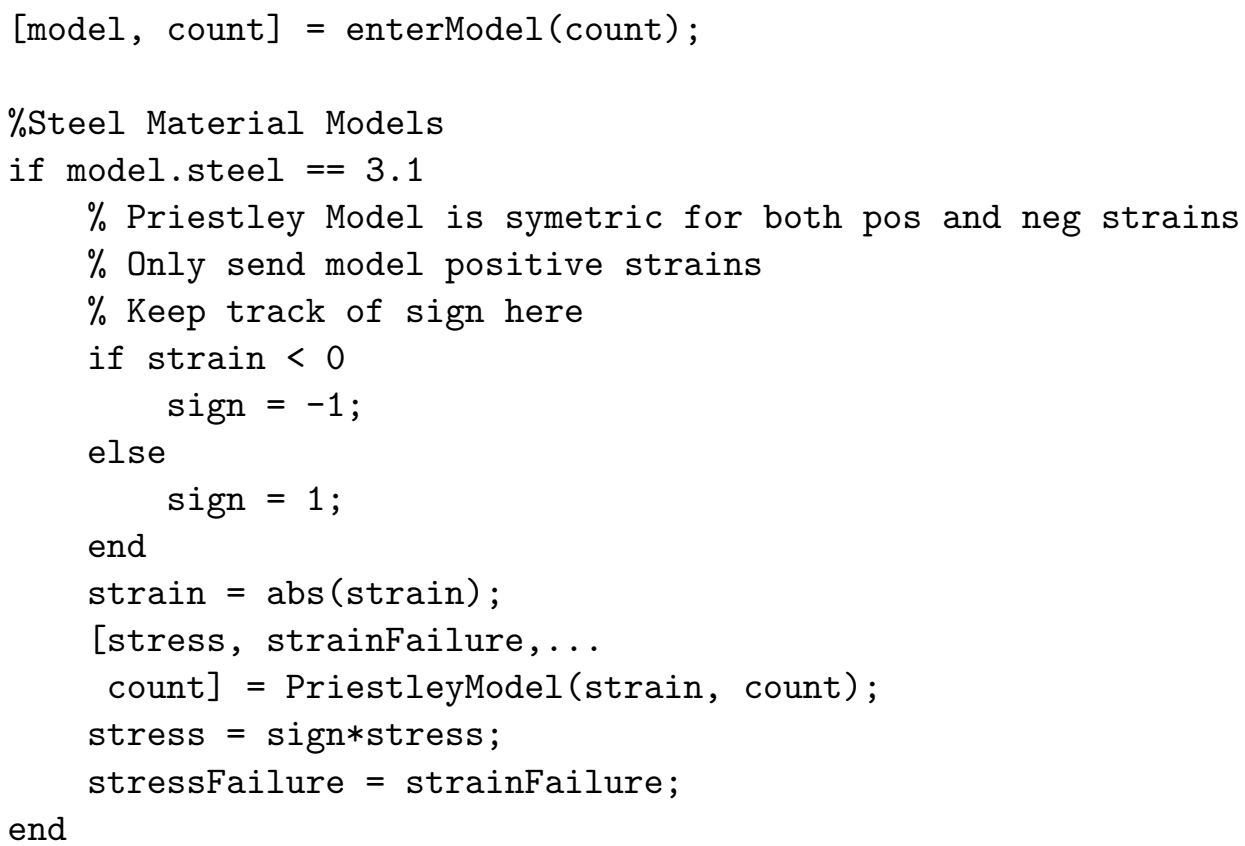

\section{B.25 Ultimate Curvature}

This function calculates the ultimate curvature of the cross section at the balanced condition.

\%Calculate Ultimate Curvature at balanced condition

function [UltimateCurvature,...

count $]=$ getUltimateCurvature (count);

[LayerProps, CoverLayerProps, BarLayerProps,...

count] = getSectionProperties (count);

[limitStrains, count] = getLimitStrains (count);

$\%$ Find location of extreme compression fiber

$\mathrm{C}=\operatorname{size}$ (LayerProps);

for $i=1: C(1)$

concCenterLines $(i, 1)=$ LayerProps $(i, 12)$;

end

$\operatorname{maxCompFiberLoc}=\max ($ concCenterLines $)+\ldots$

LayerProps $(1,14) / 2$;

$\%$ Find Location of extreme tension fiber 


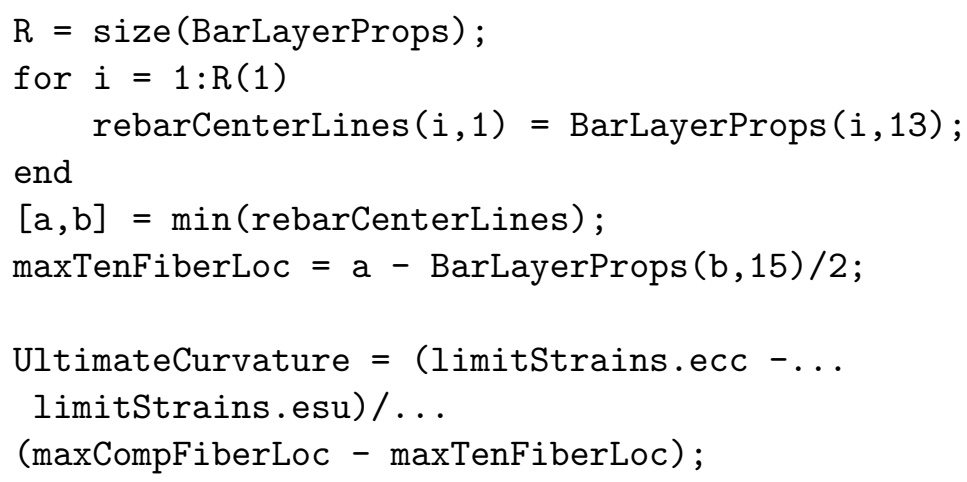

\section{B.26 PM Interaction Diagram}

This function calculates and plots points on PM Interaction diagrams.

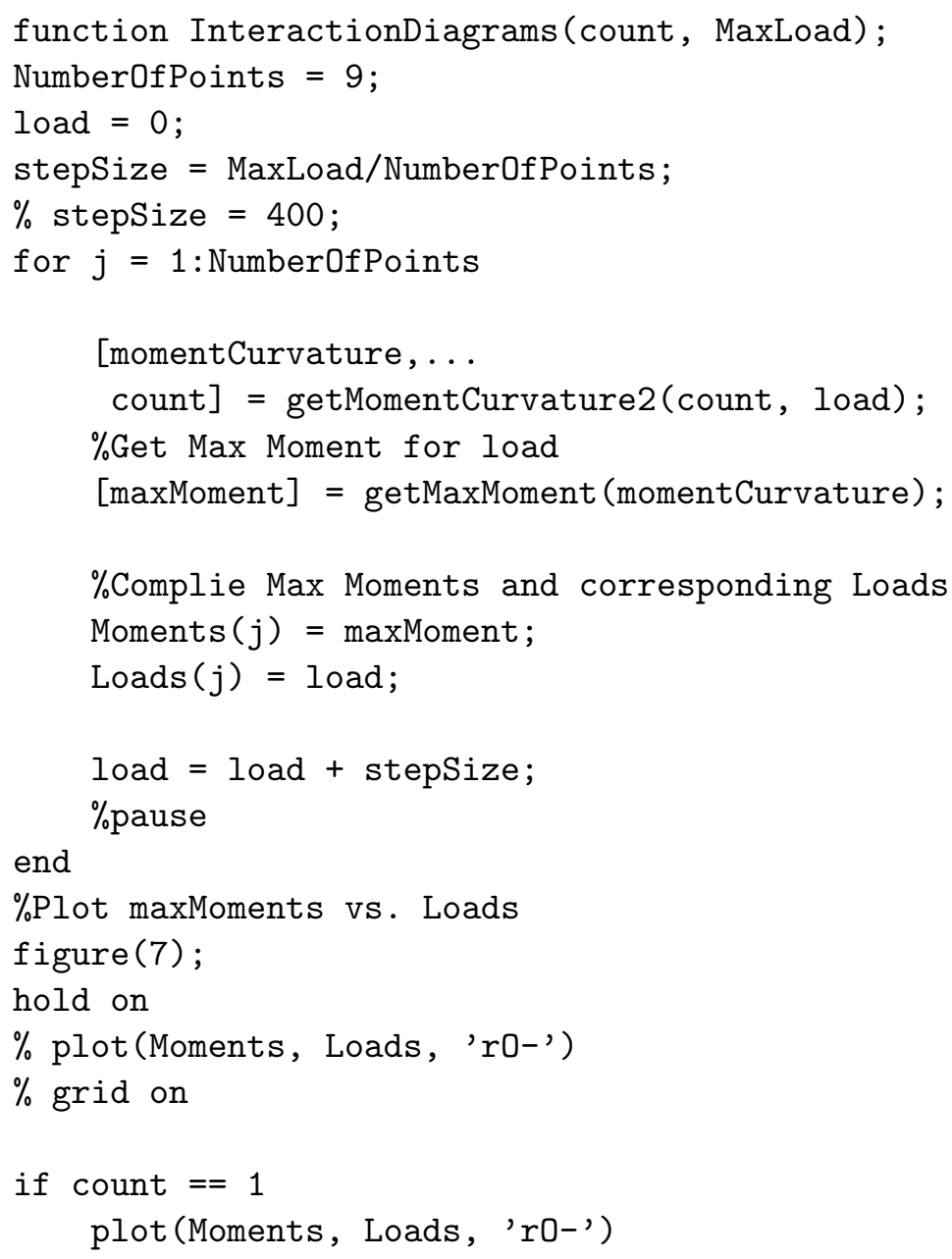




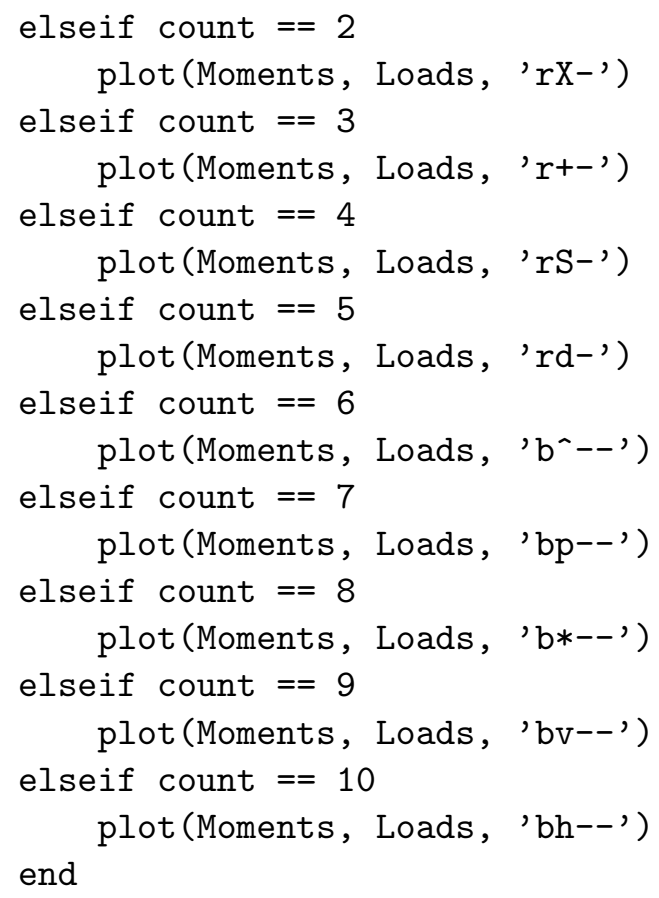

\section{B.27 Load Check}

This function ensures the applied axial load does not exceed the cross section's allowable load.

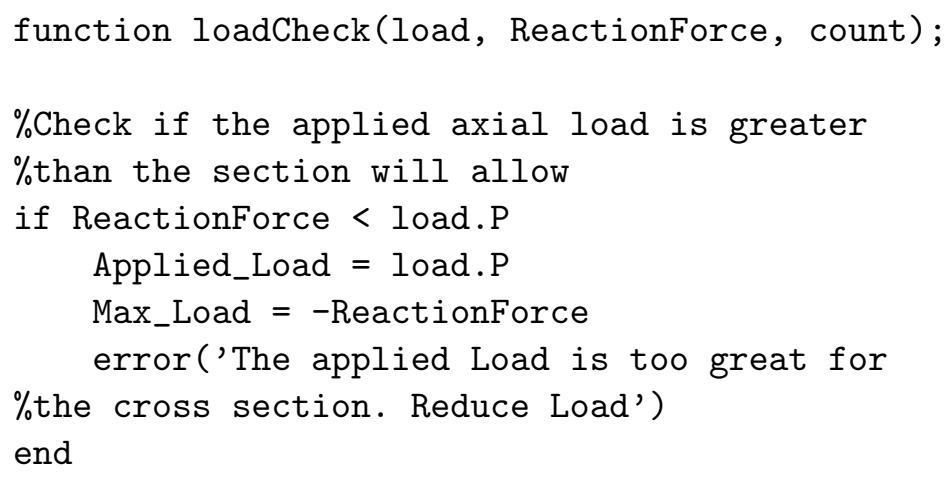




\section{B.28 Mander Confined Concrete Material Model}

This function calculates stress at a specified strain per the Mander Confined Con-

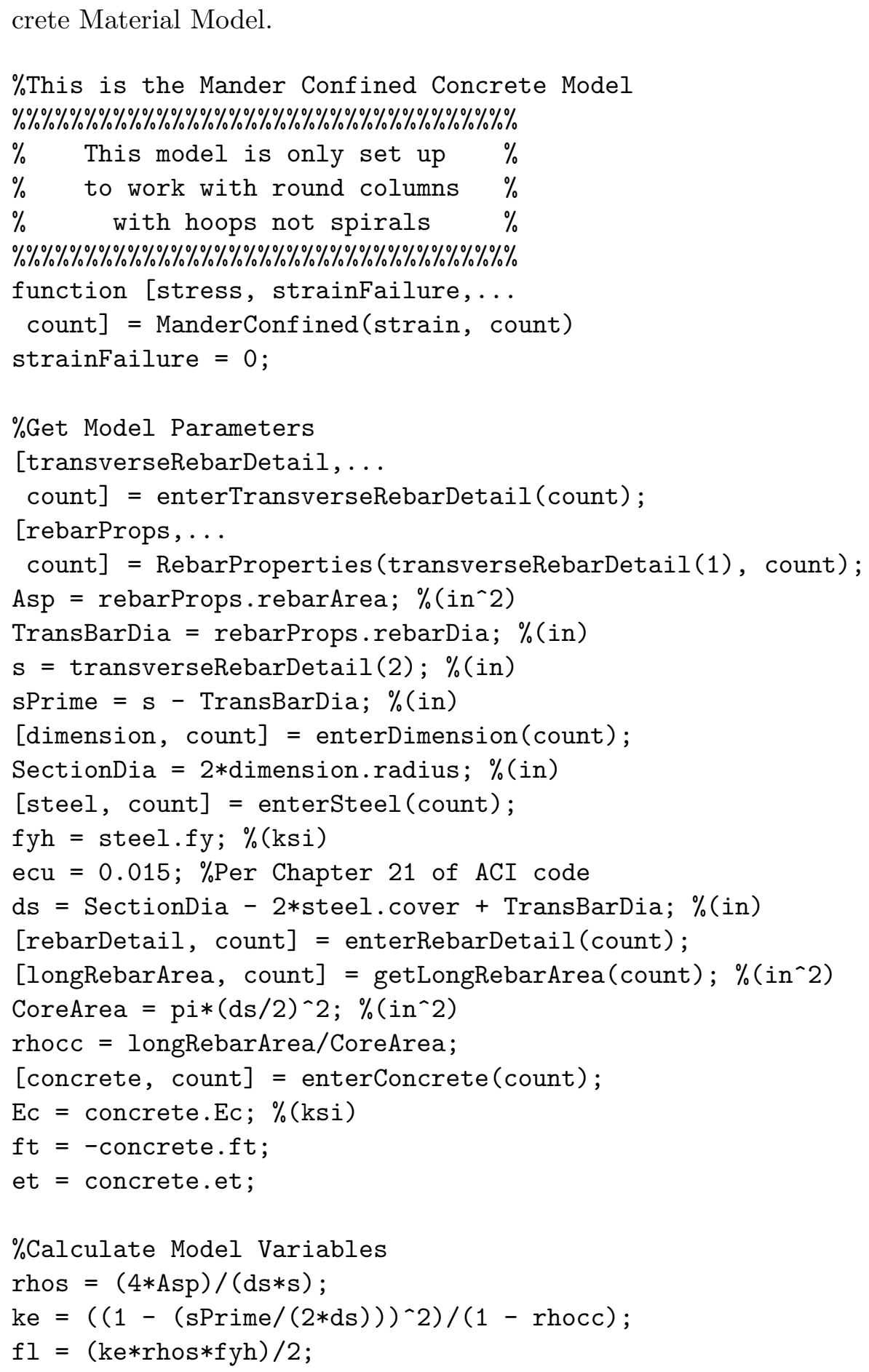




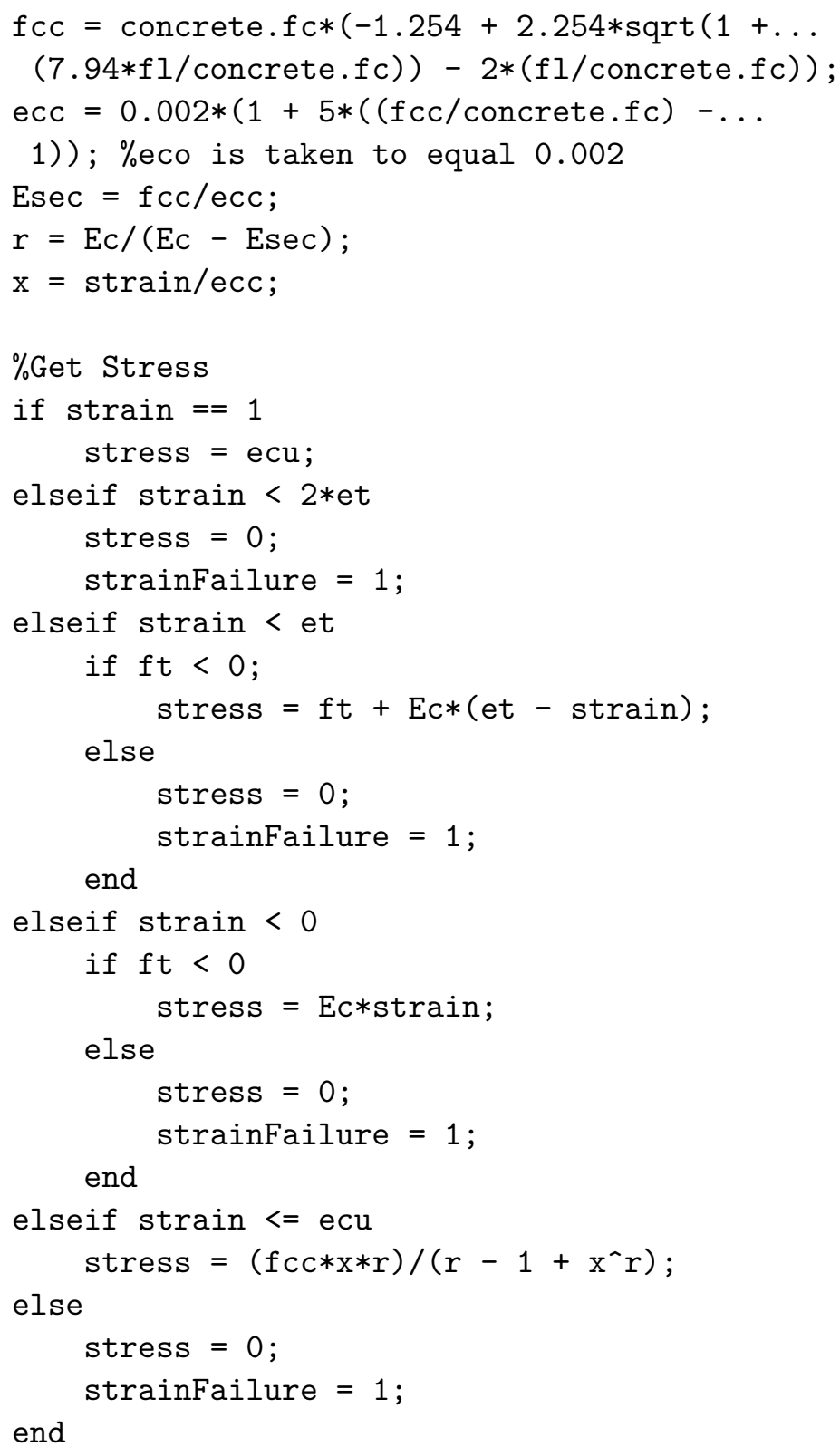

\section{B.29 Mander Unconfined Concrete Material Model}

This function calculates stress at a specified strain per the Mander Unconfined Concrete Material Model.

$\%$ This is the Mander unconfined concrete model 


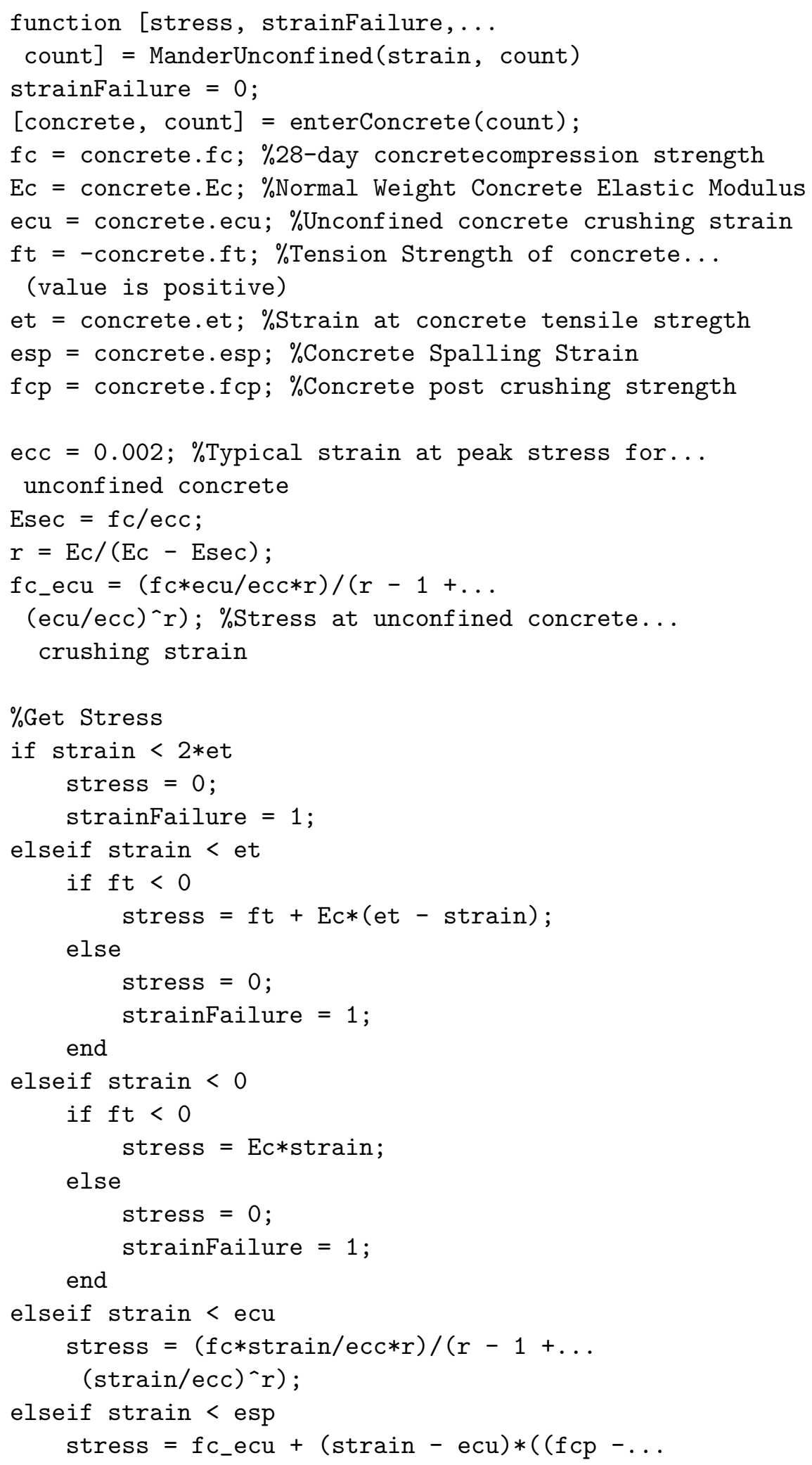




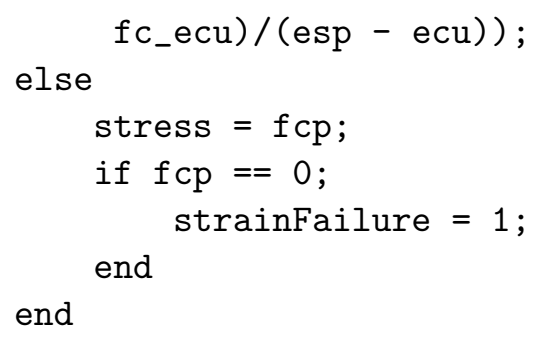

\section{B.30 Moment-Curvature Plot}

This function plots the Moment-Curvature diagrams.

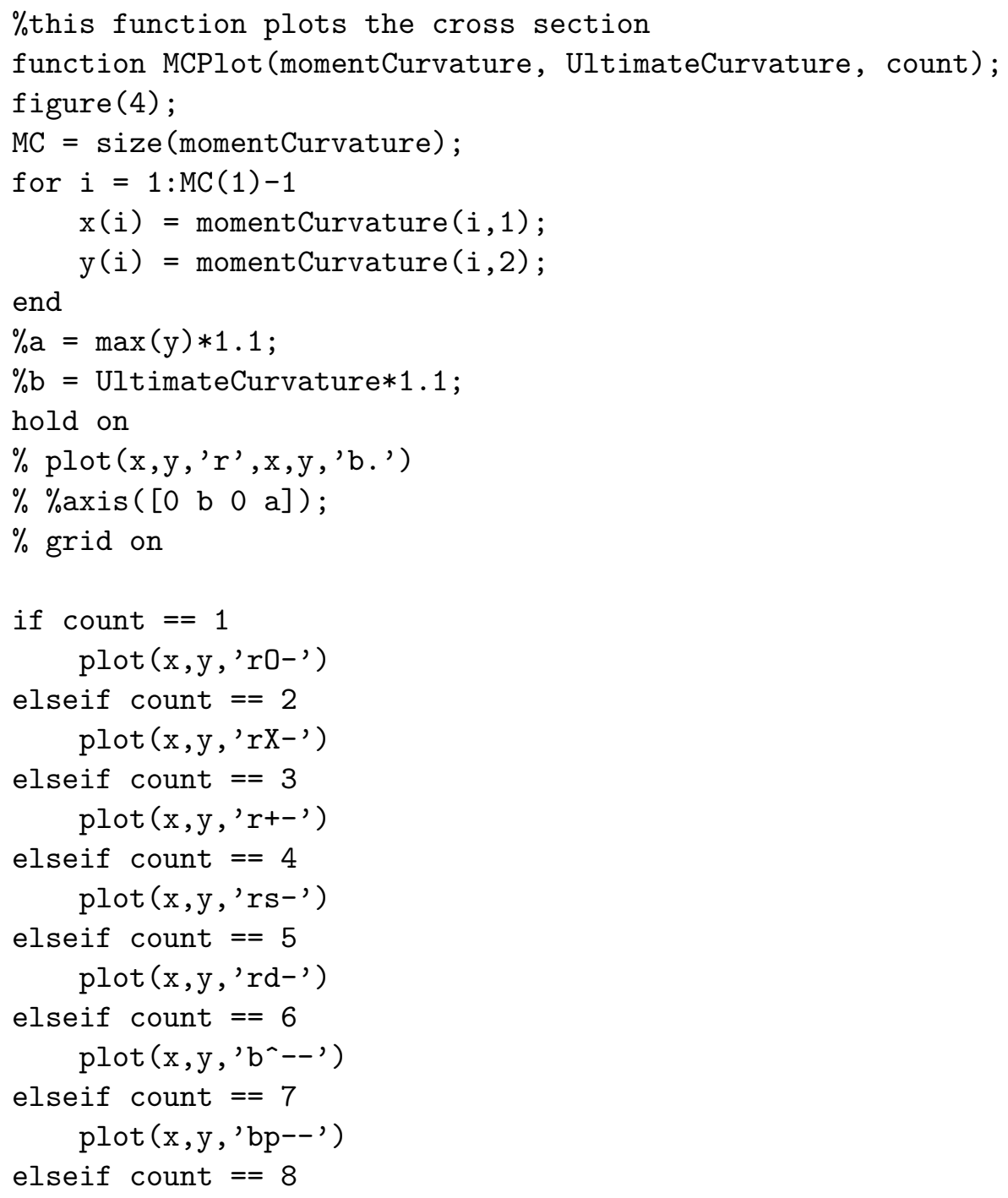




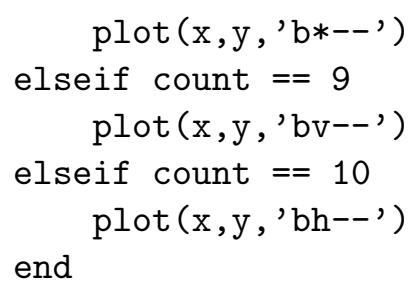

\section{B.31 Material Model Stress-Strain Plots}

This function plots the stress-strain curves for various material models.

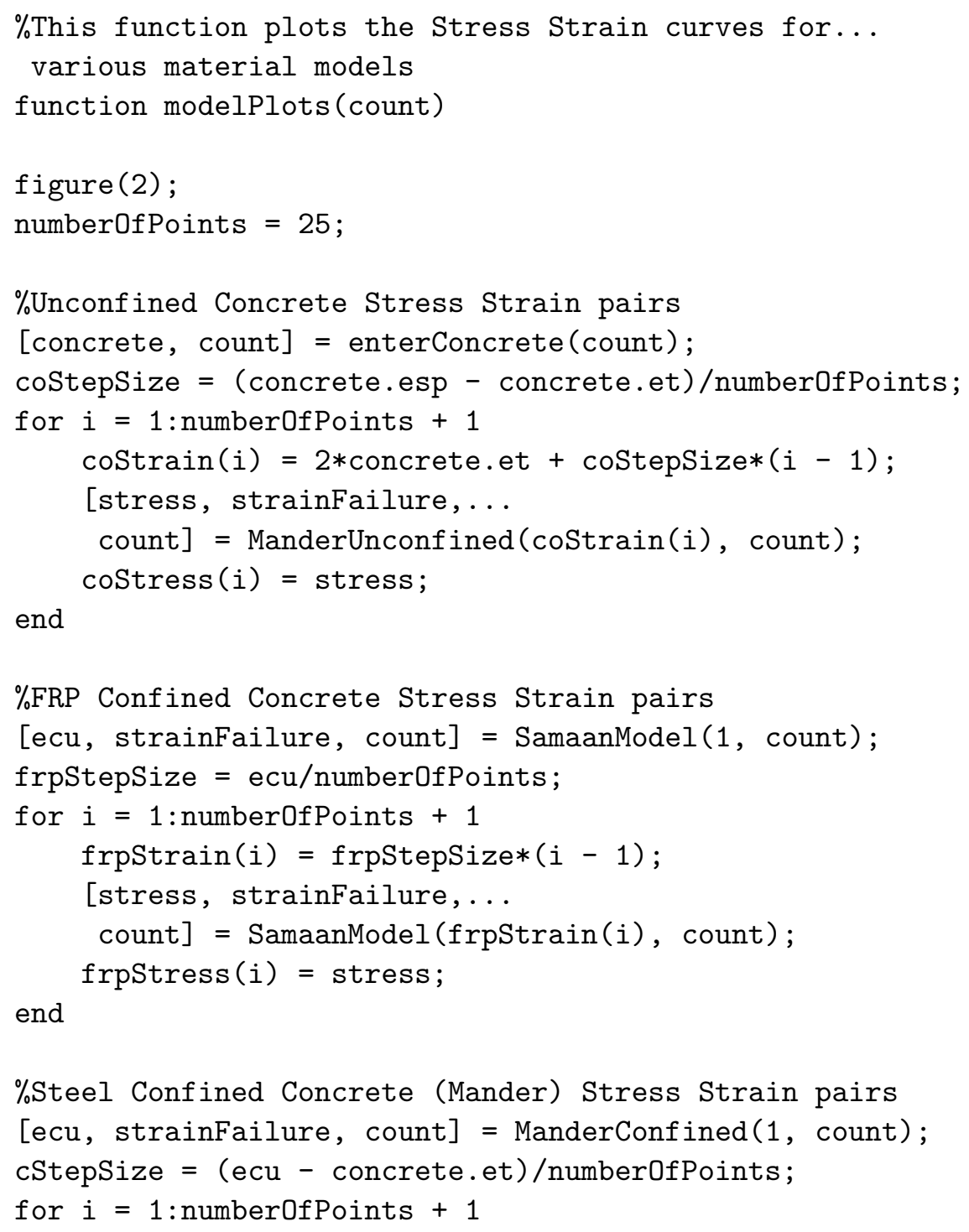




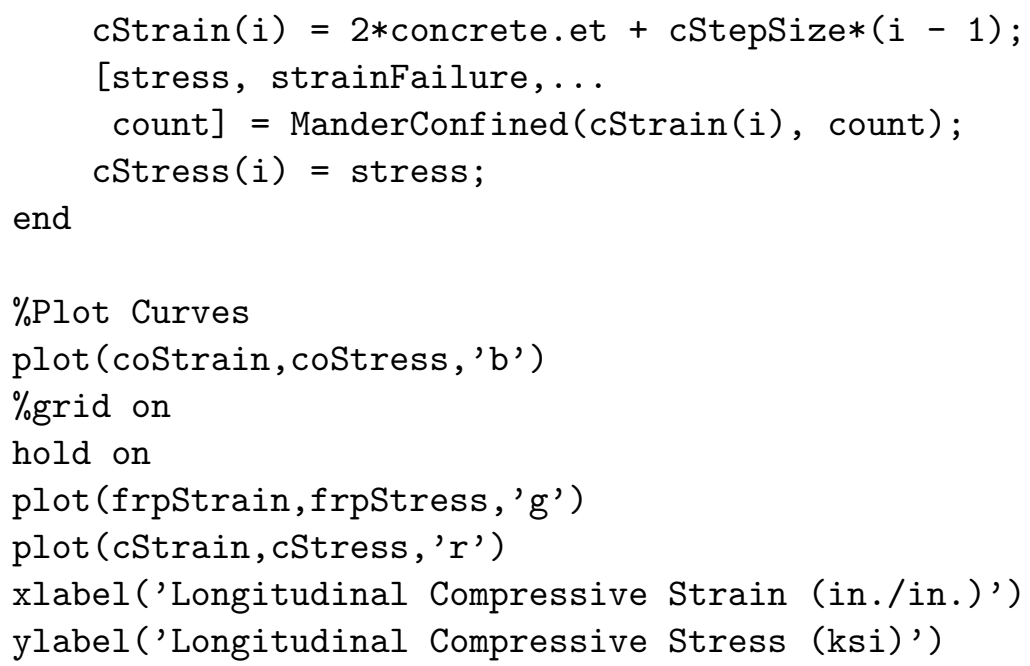

\section{B.32 Priestley Bi-Axial Steel Model}

This function calculates stress in at a specified stain per the Priestley Bi-Axial Steel Model.

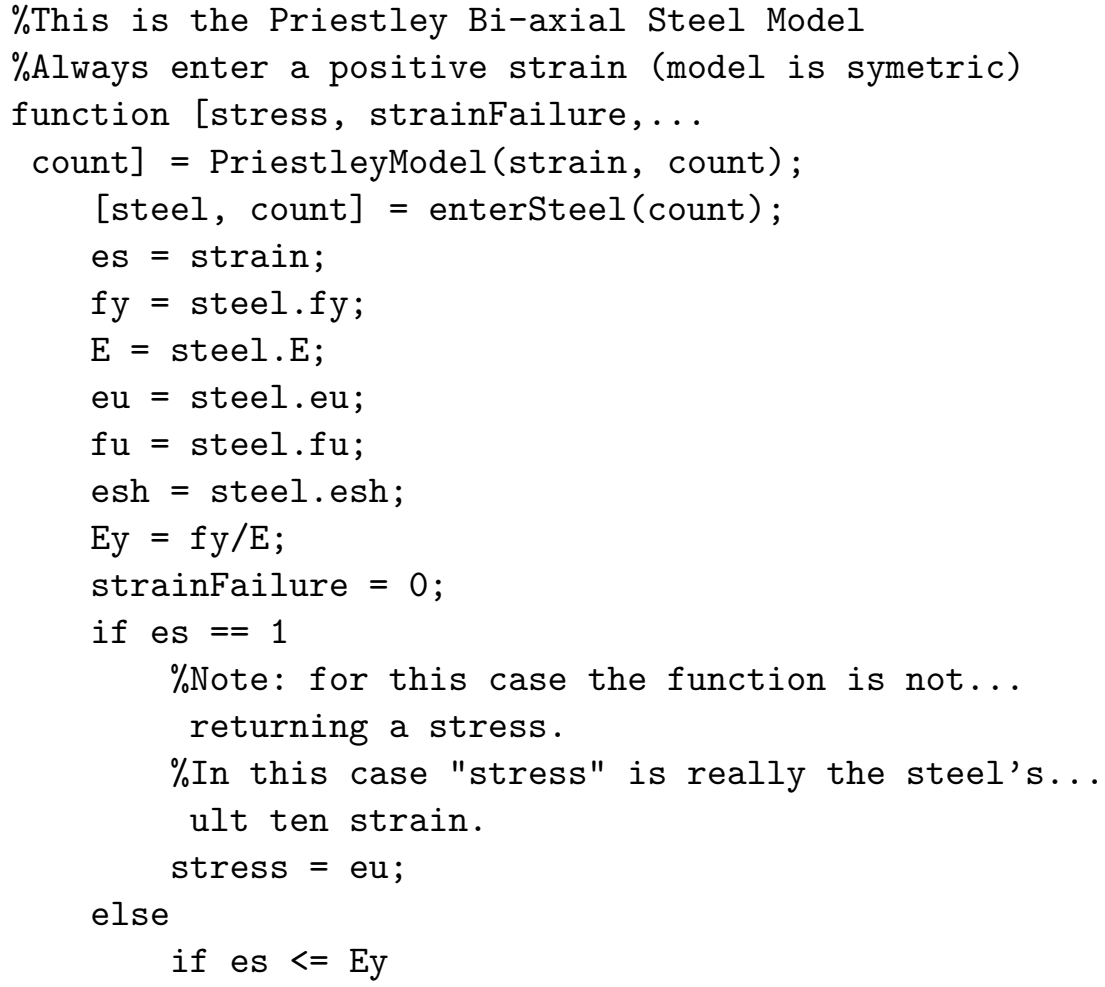




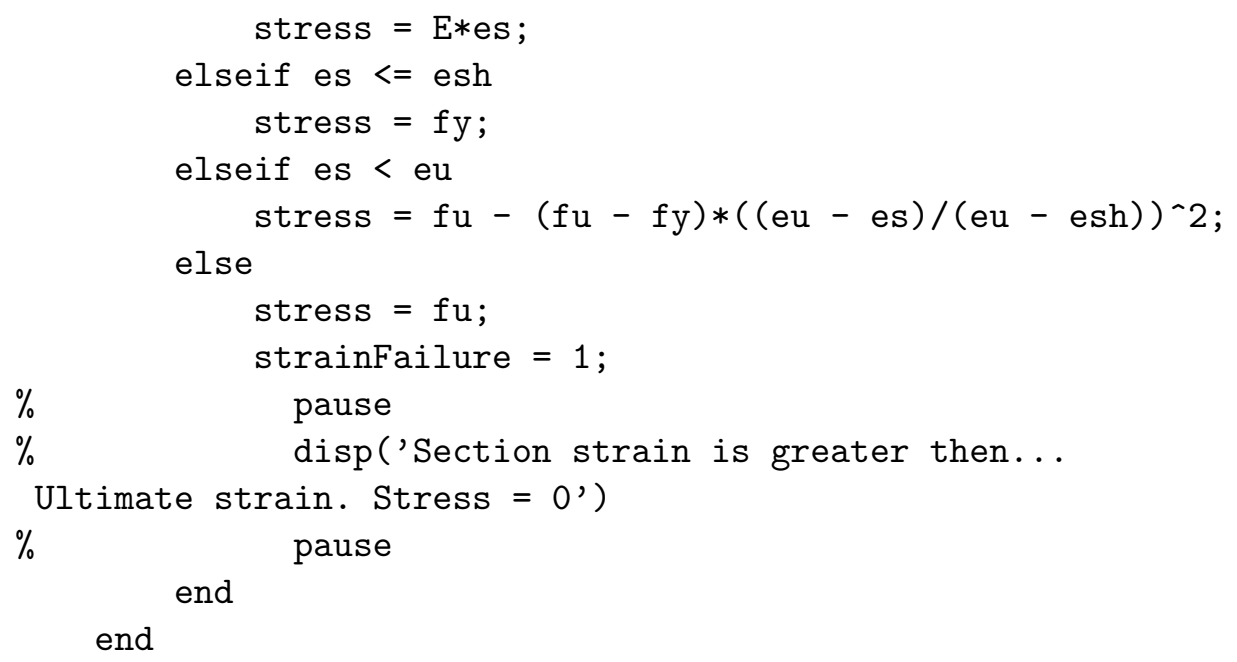

\section{B.33 Rebar Properties}

This function provides dimensional properties of rebar for a specified bar size.

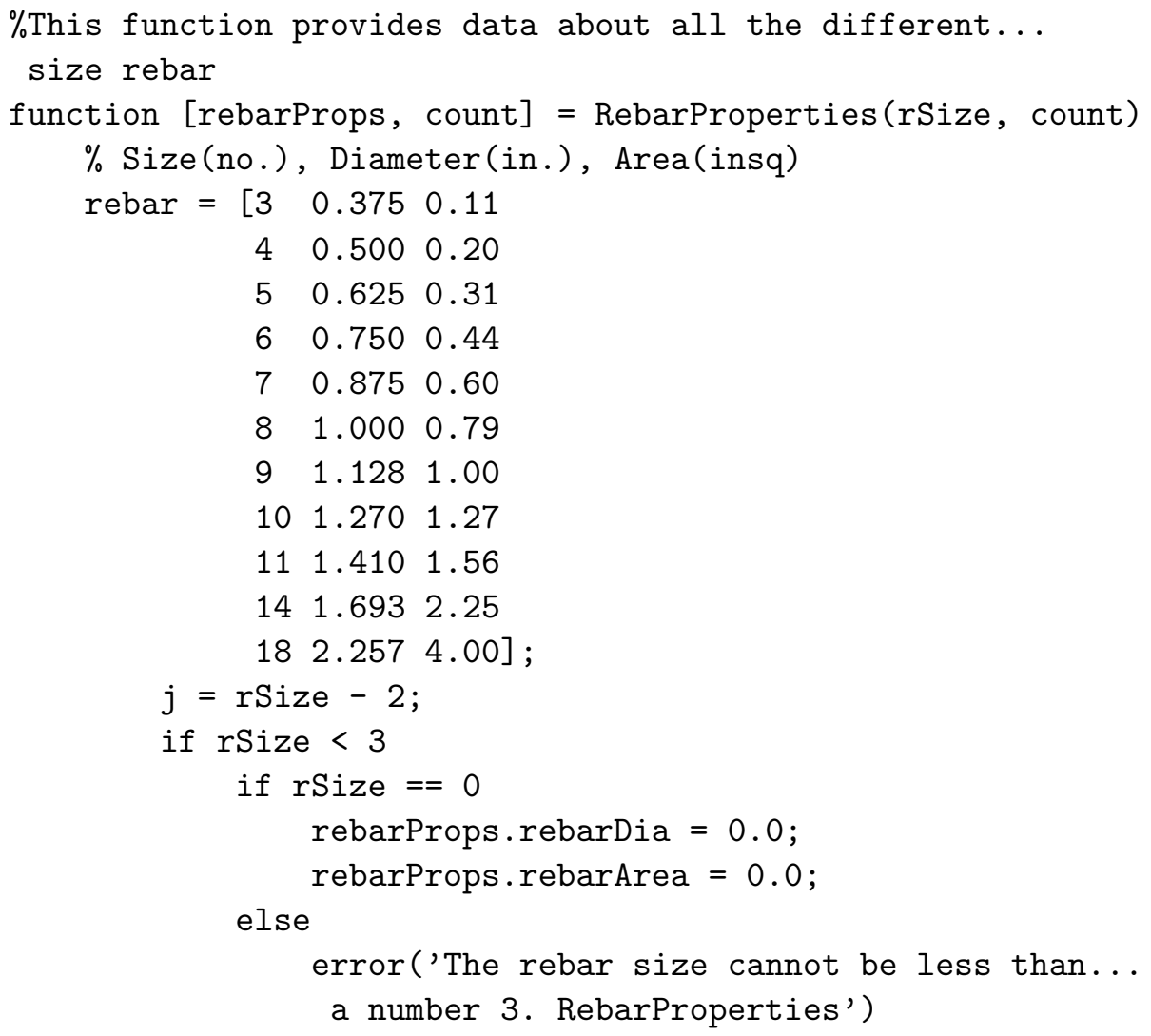


end

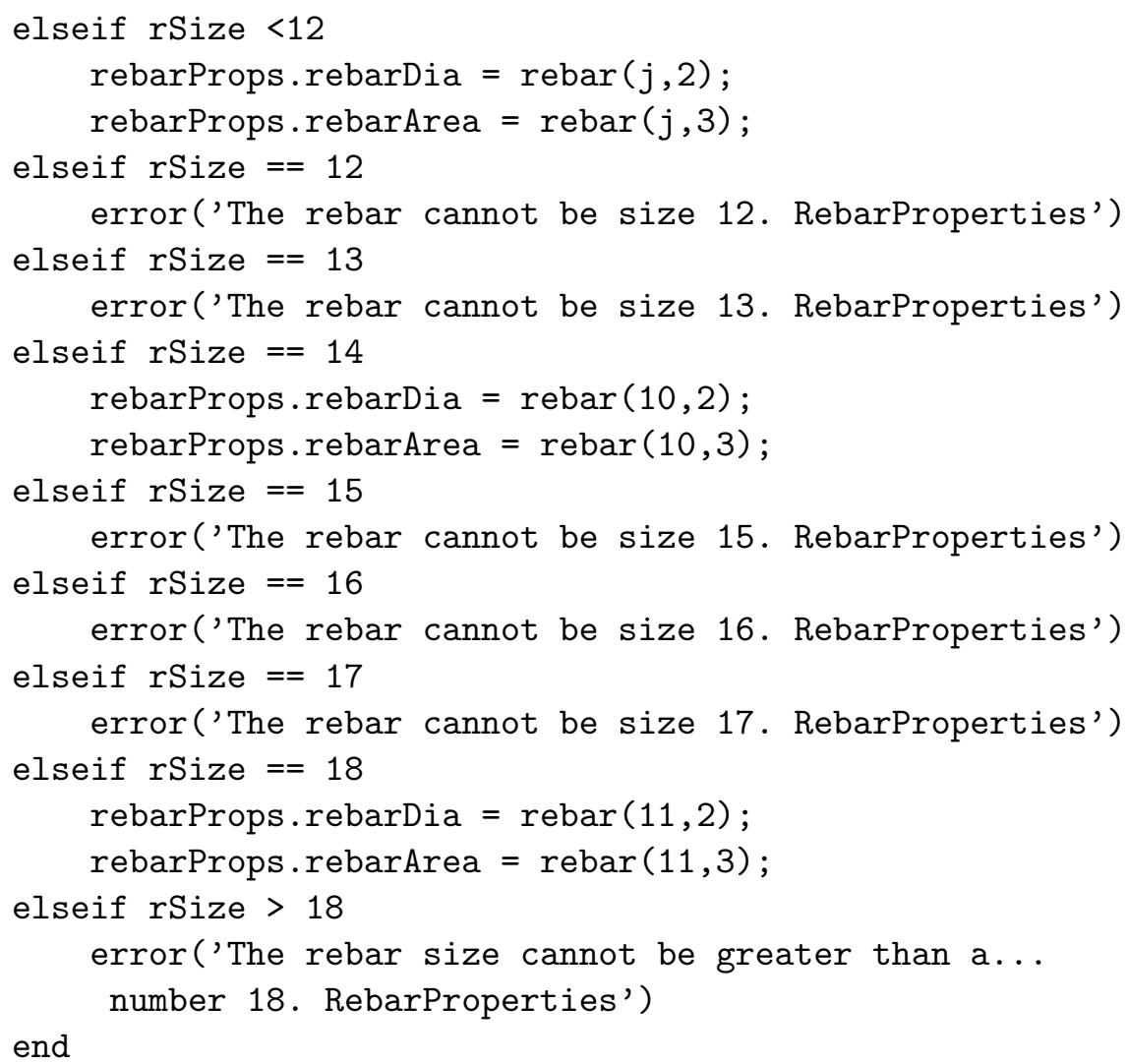

\section{B.34 Samaan Confined Concrete Material Model}

This function calculates stress in at a specified stain per the Samaan Confined Concrete Material Model.

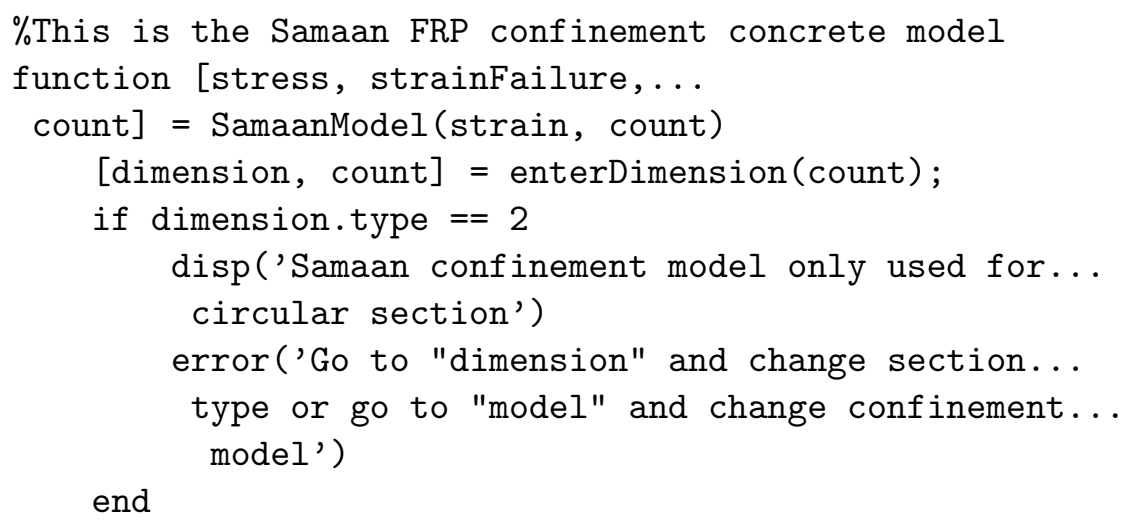




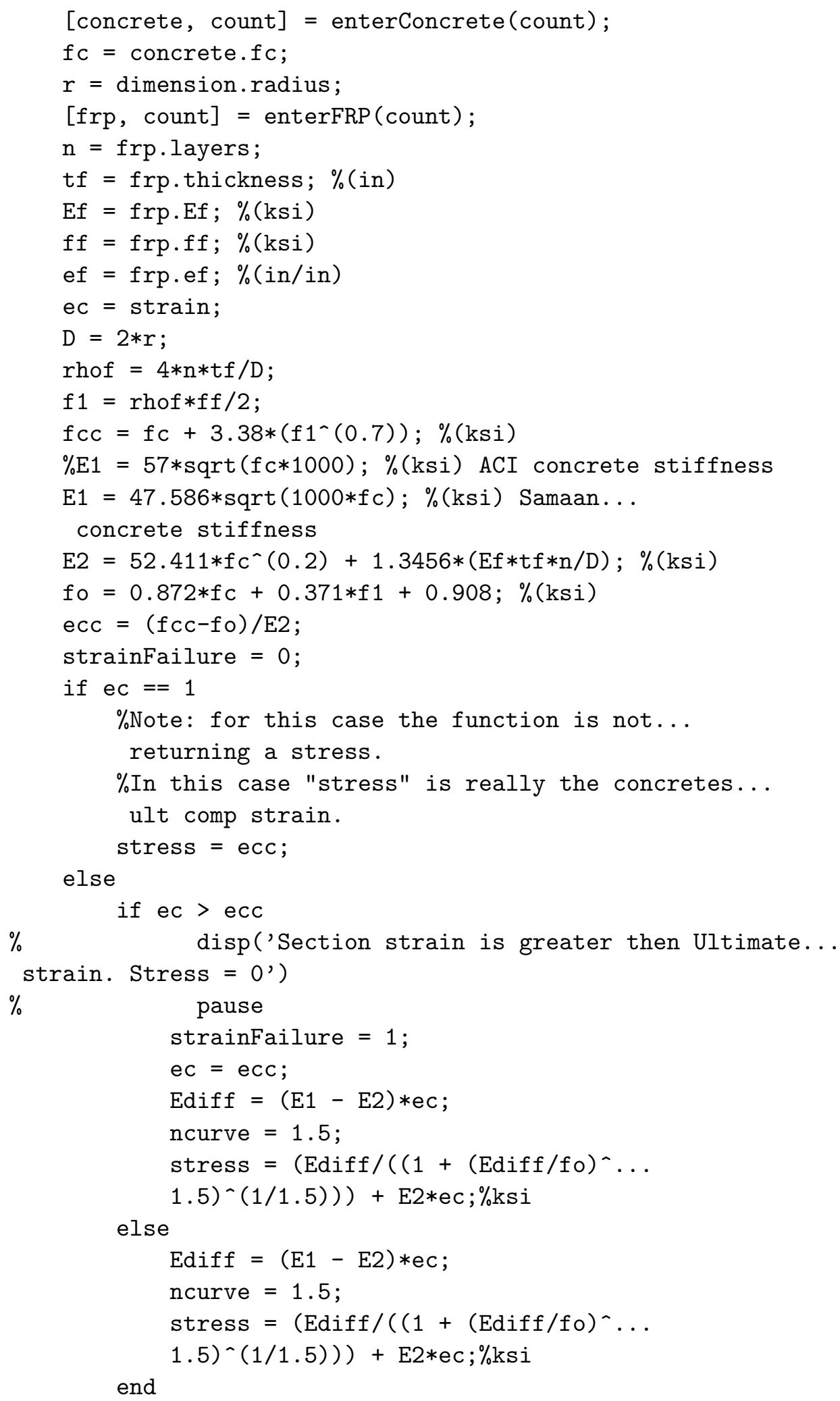


end

\section{B.35 Section Plot}

This function plots the discretized cross section.

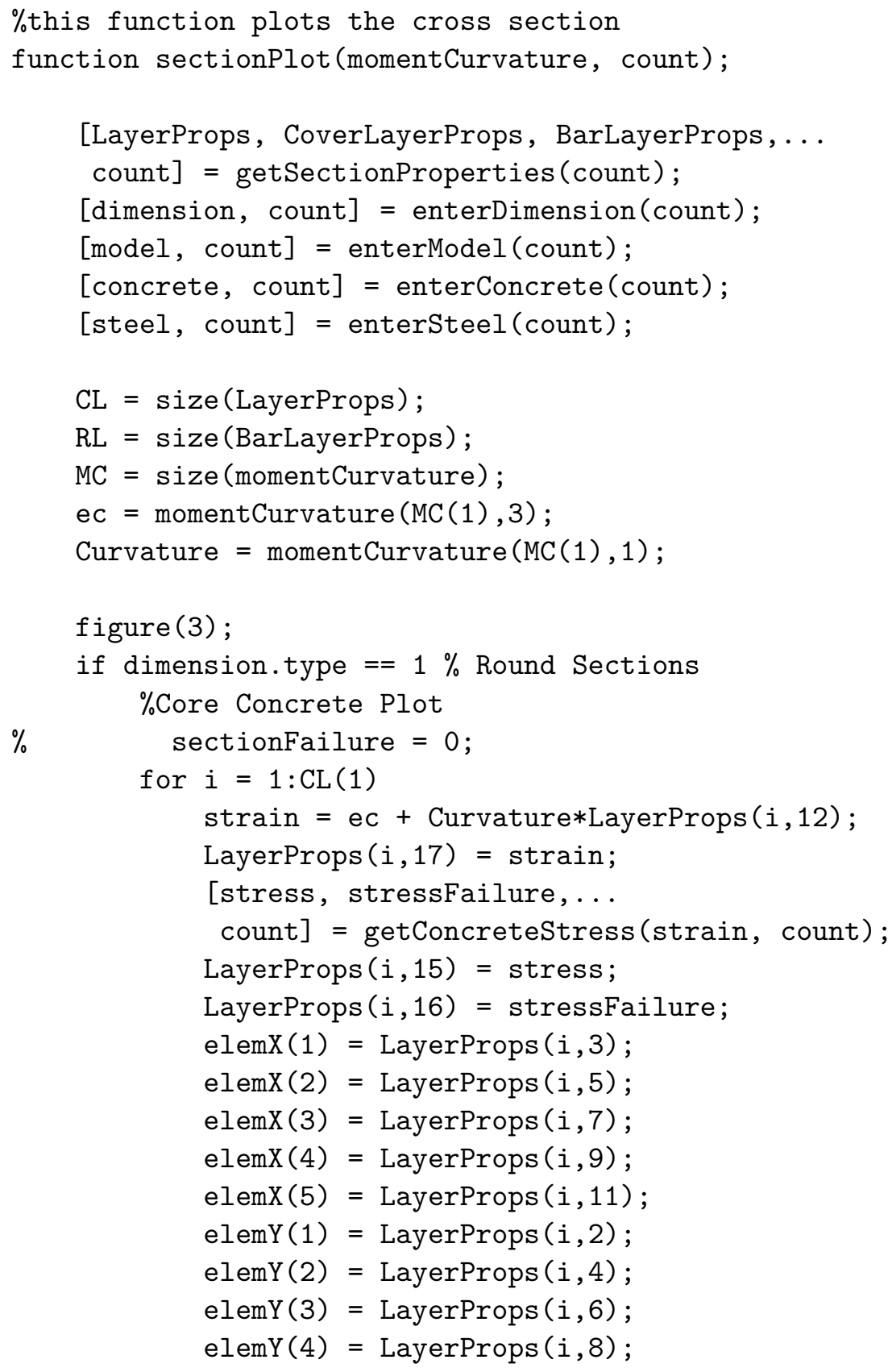




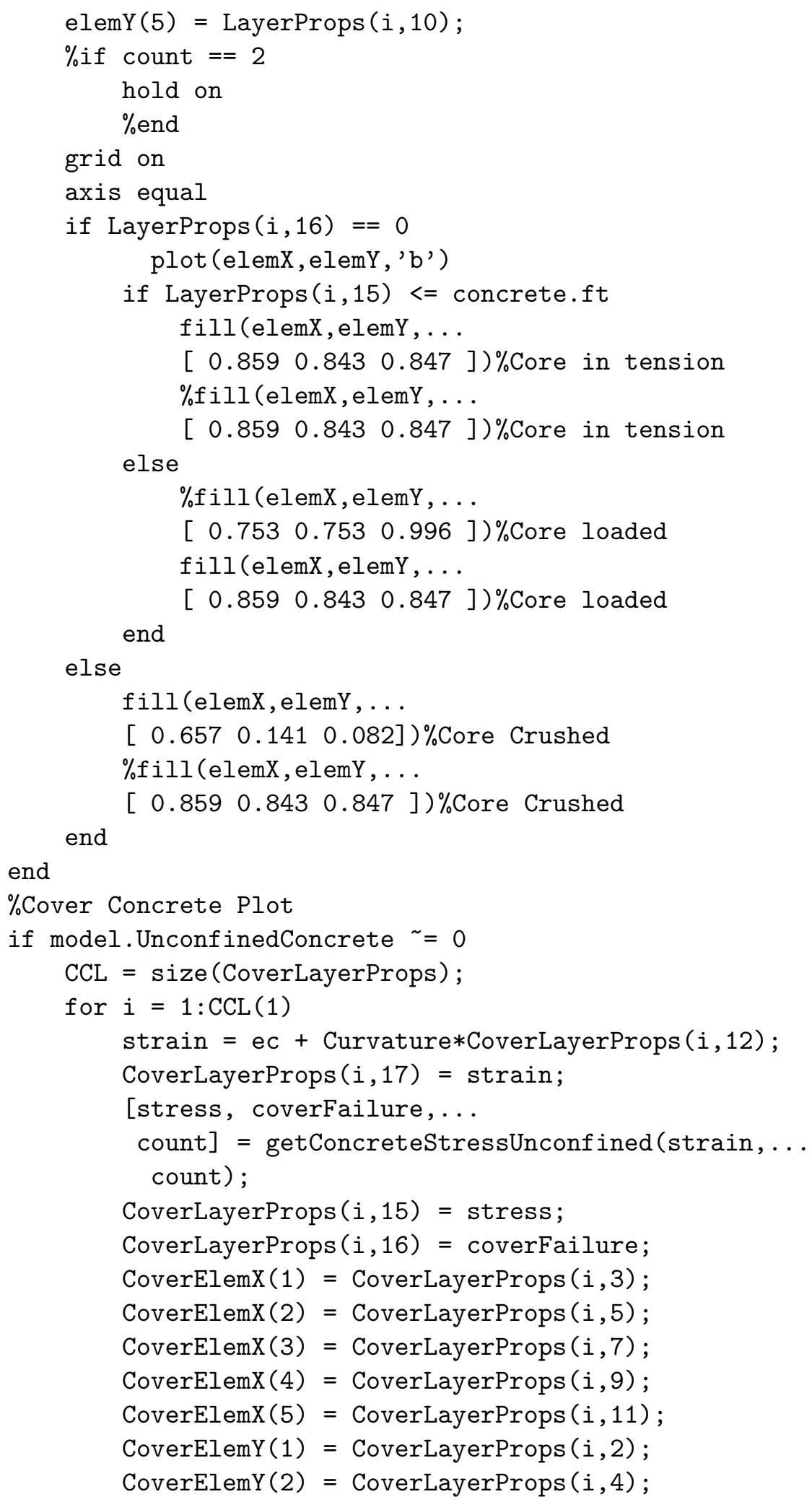




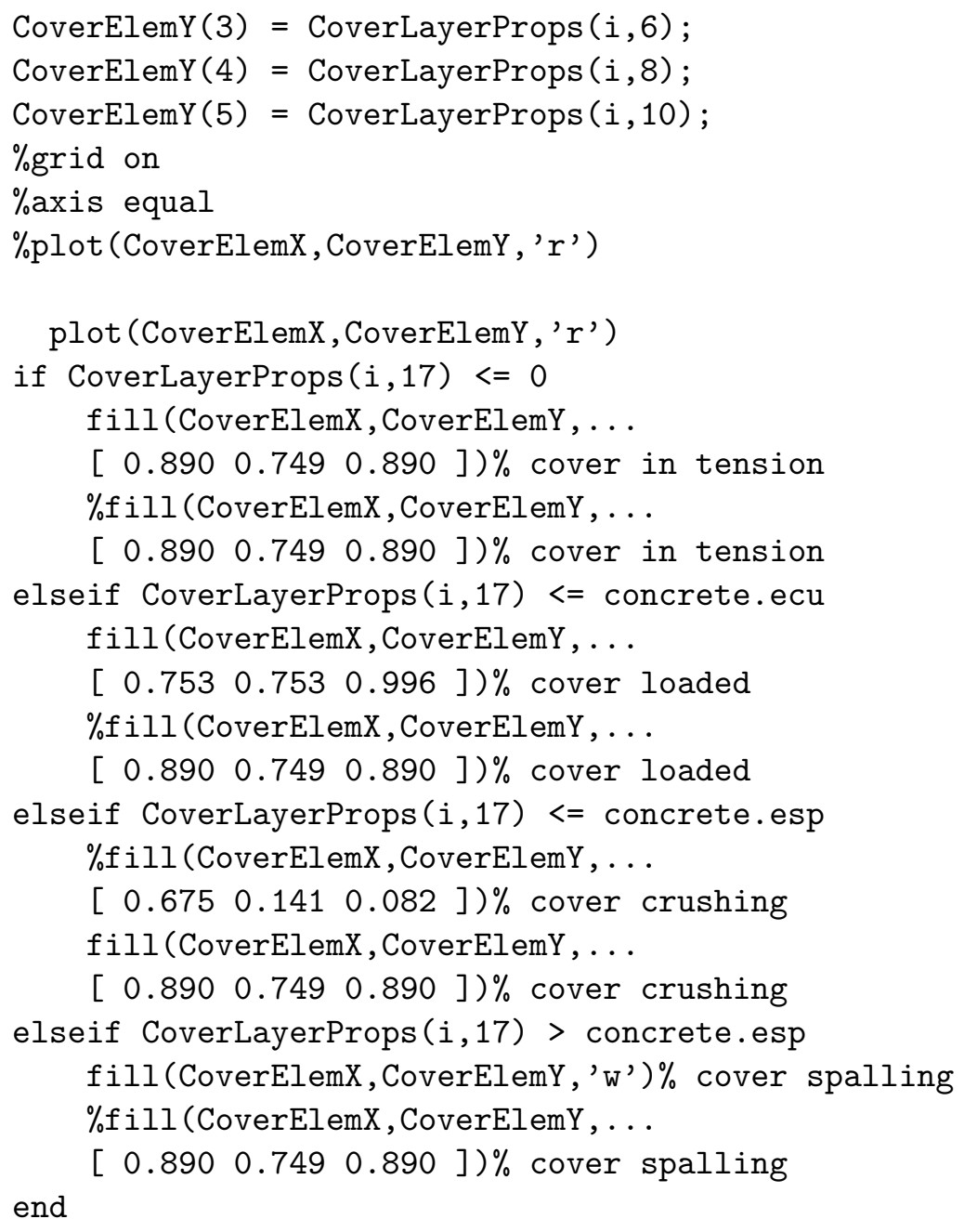




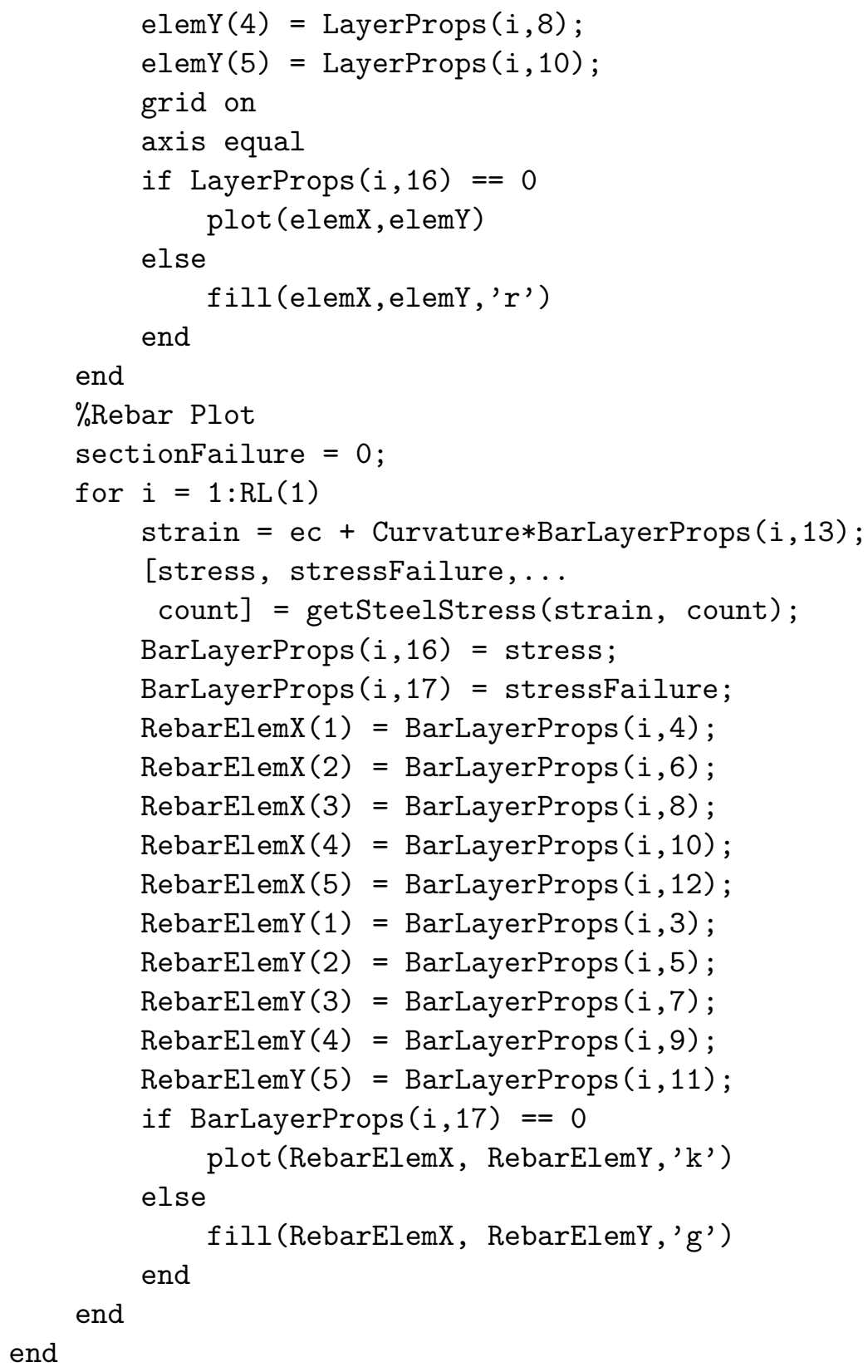

\section{B.36 FRP and Steel Material Stress-Strain Plots}

This function plots the stress-strain material curves for FRP and Steel.

\%This function plots the Stress Strain Curve for... FRP and Steel 


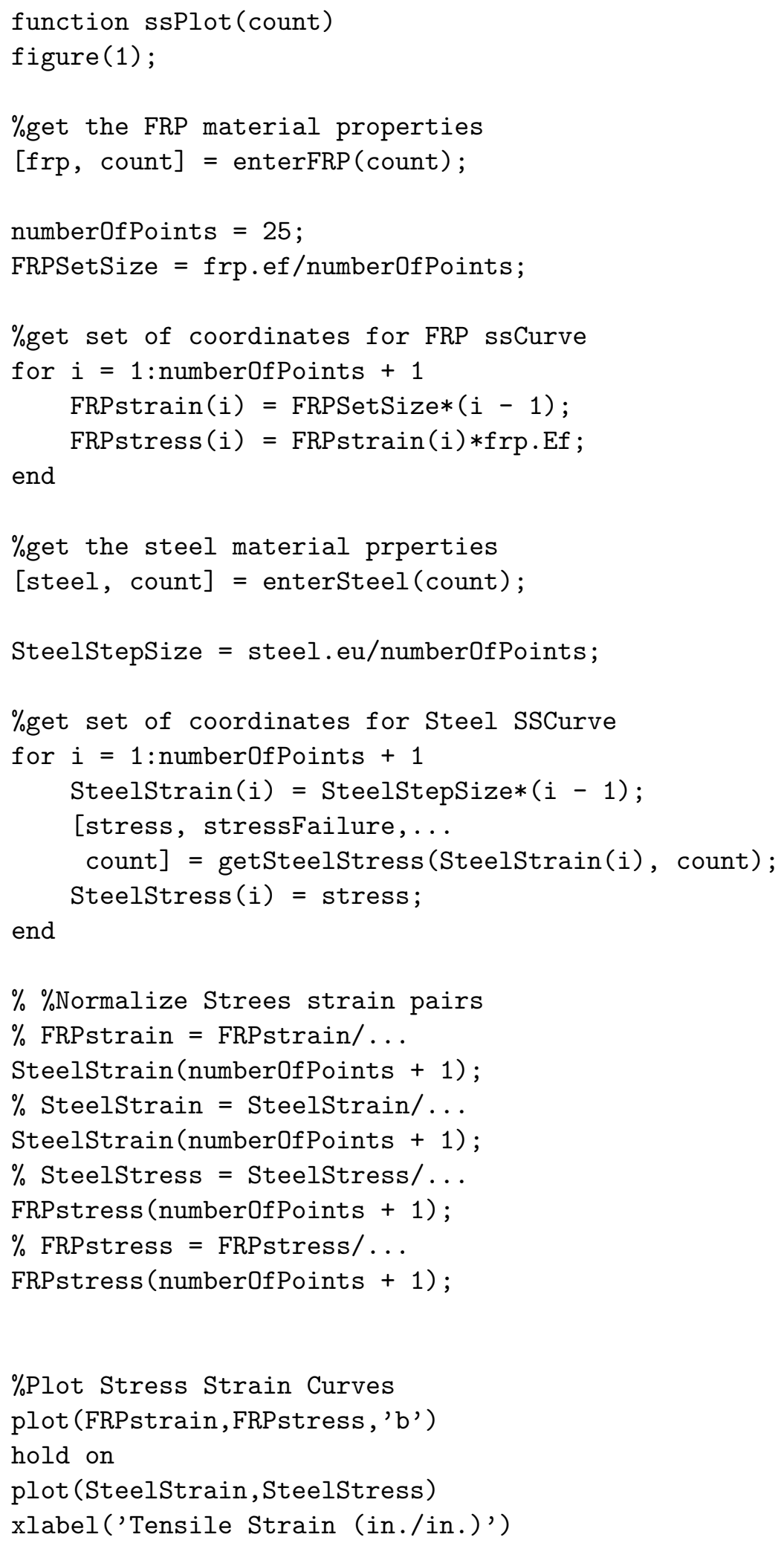


ylabel('Tensile Stress (ksi)')

\section{B.37 Cross Section Failure Strain Plot}

This function plots the strains throughout the cross section at failure.

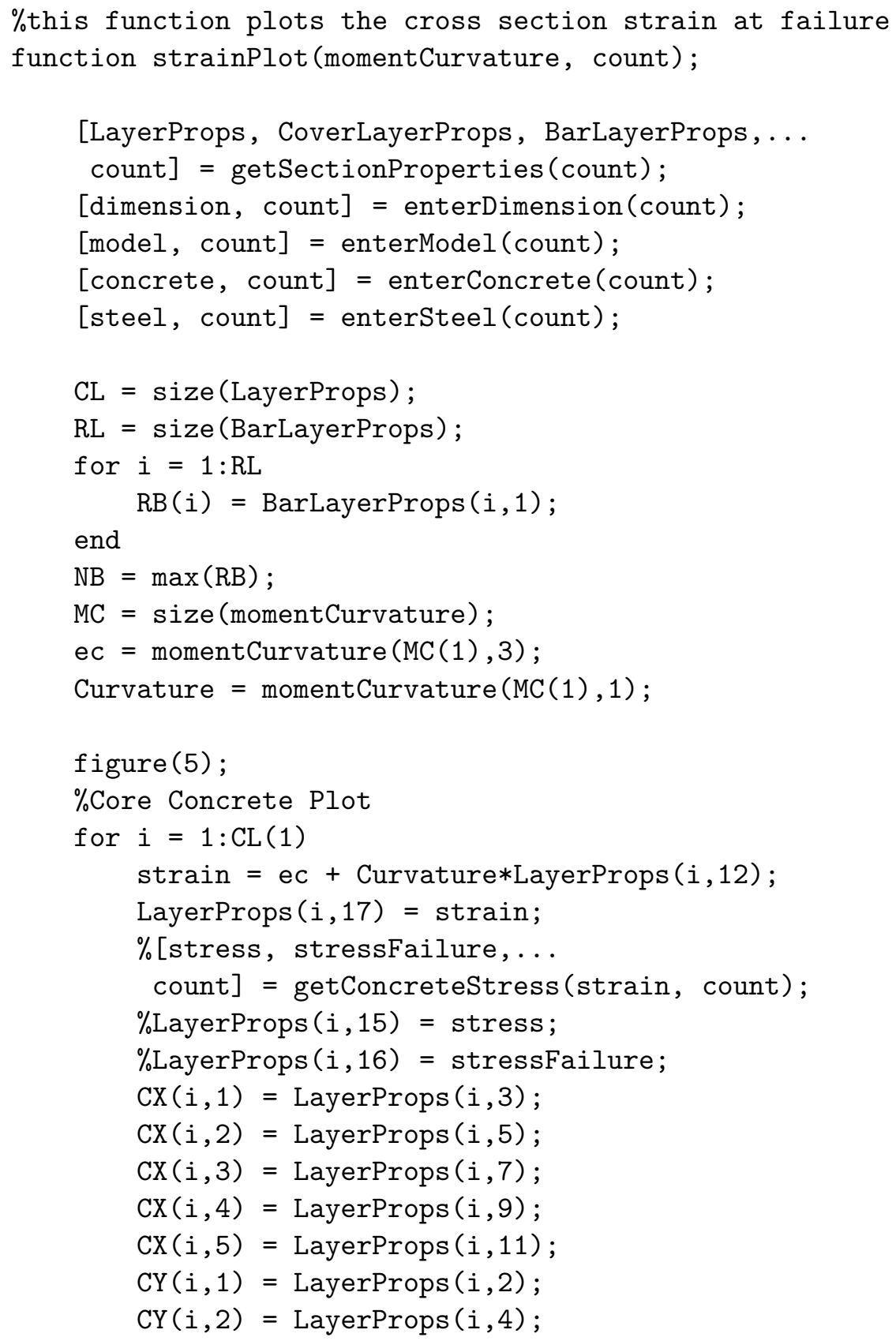




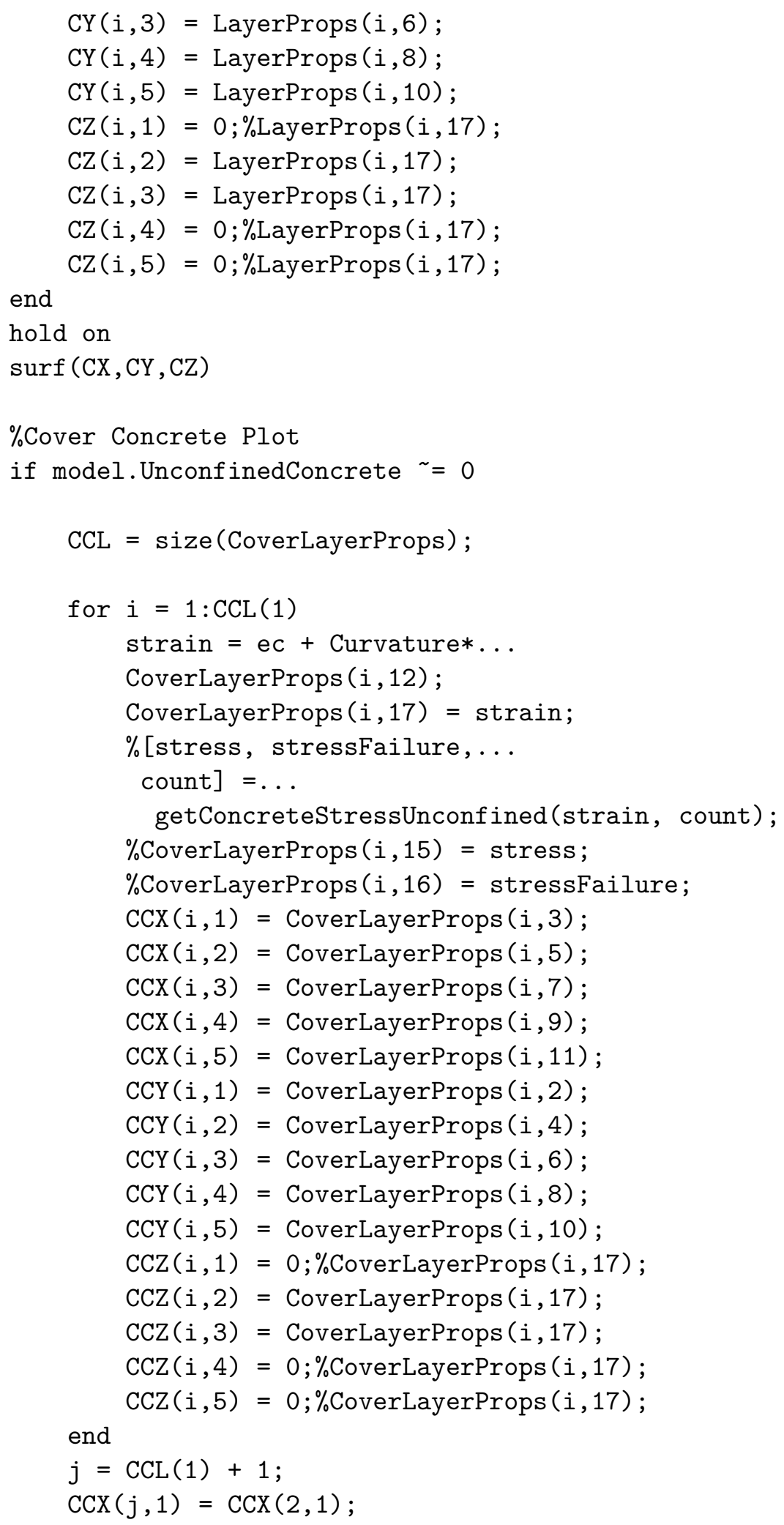




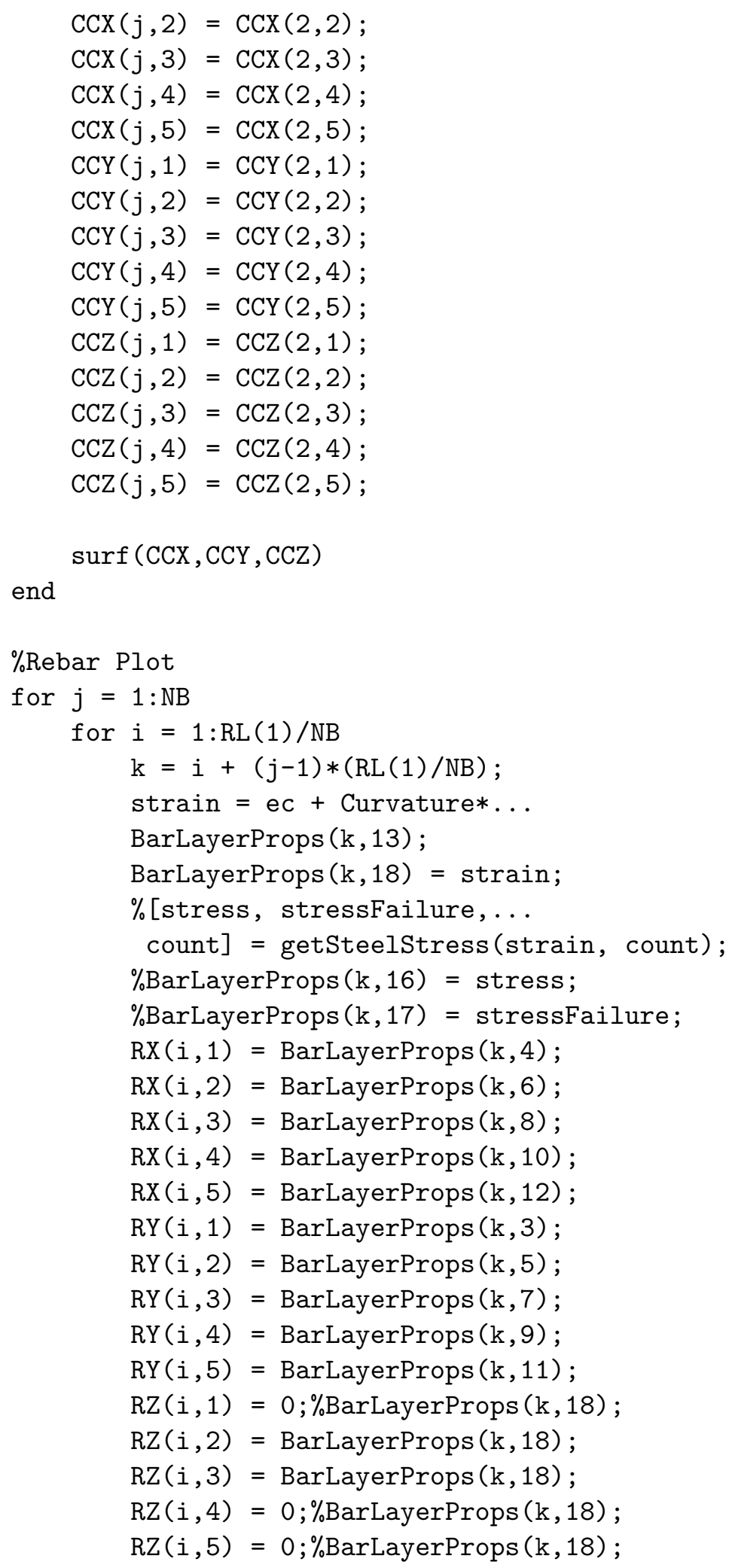


end

$\operatorname{surf}(R X, R Y, R Z)$

end

\section{B.38 Cross Section Failure Stress Plot}

This function plots the stress throughout the cross section at failure.

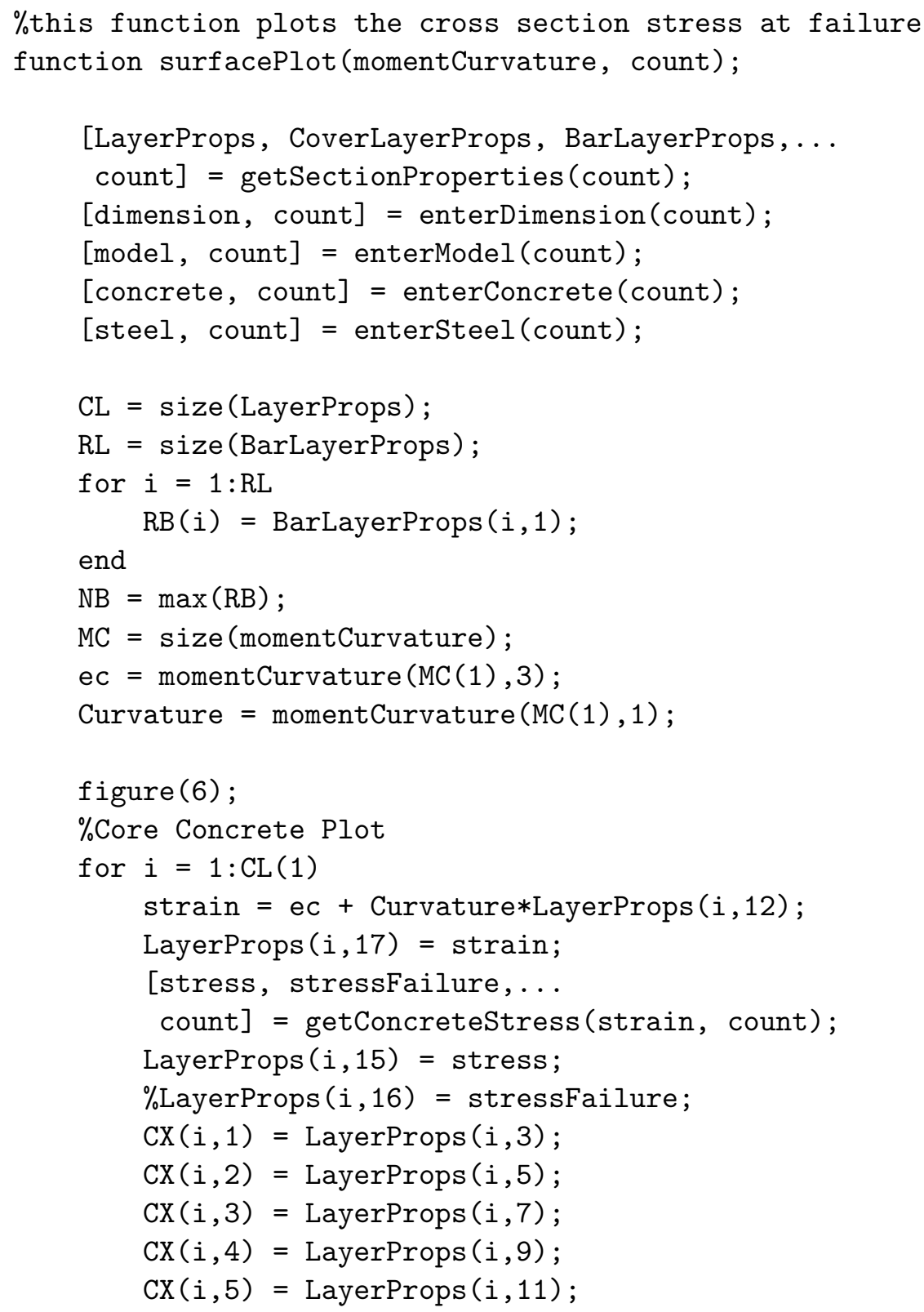




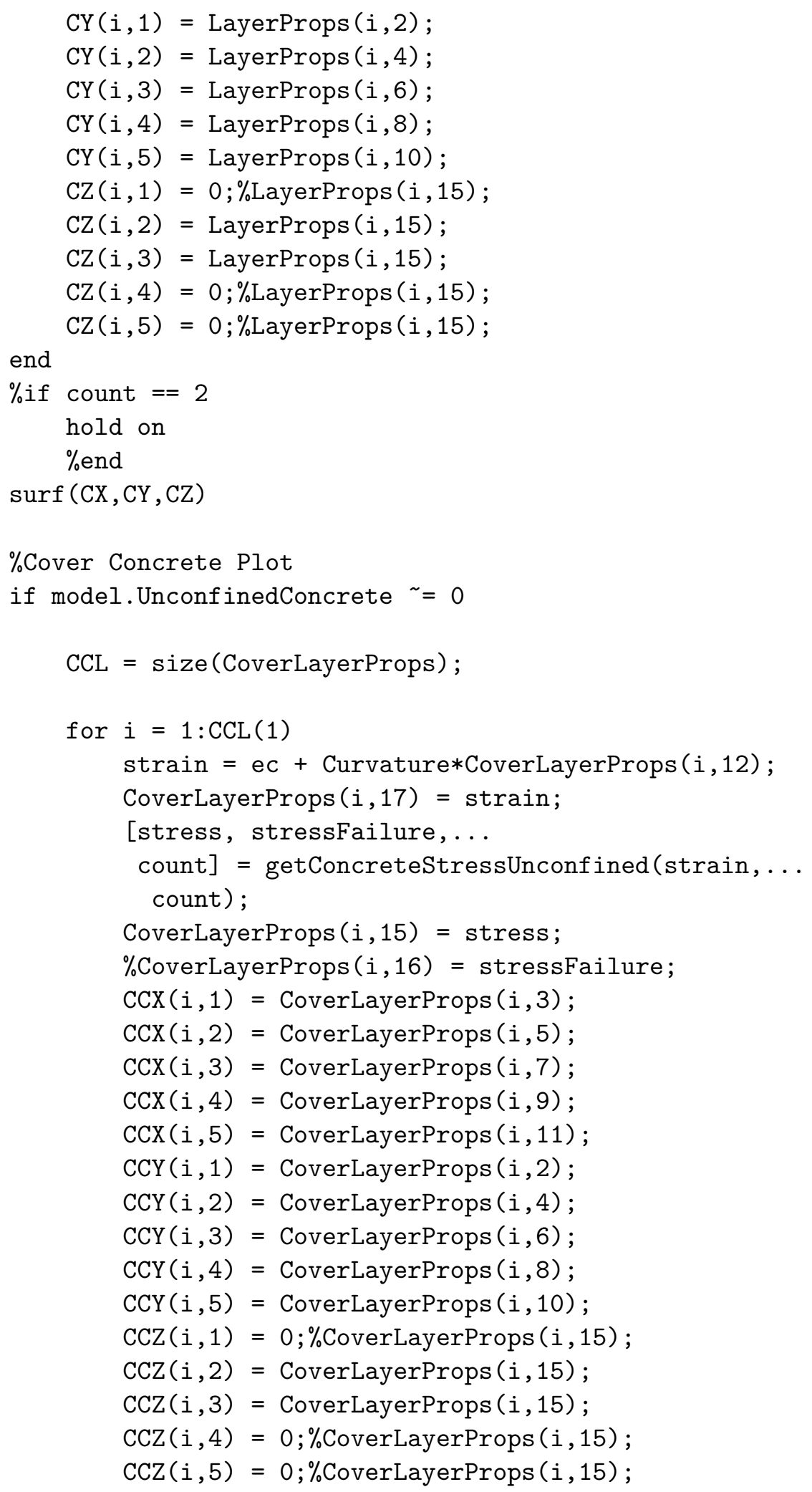




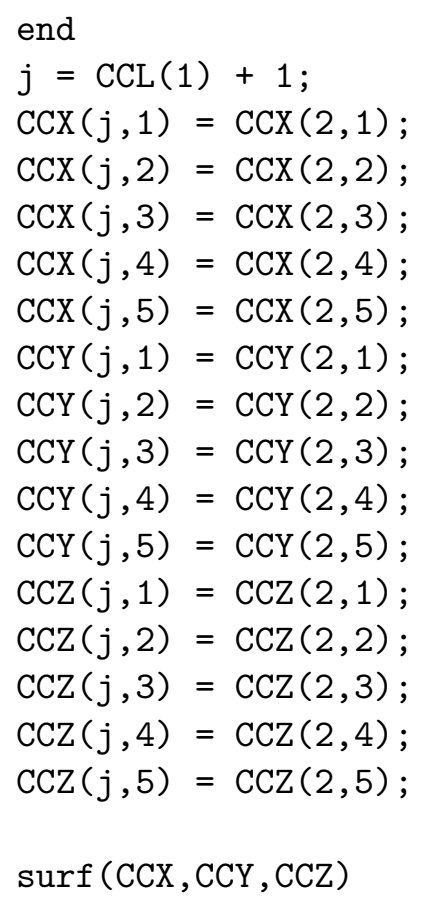


RZ $(\mathrm{i}, 4)=0 ; \%$ BarLayerProps $(\mathrm{k}, 16) ;$

$R Z(i, 5)=0 ; \%$ BarLayerProps $(k, 16)$;

end

$\operatorname{surf}(R X, R Y, R Z)$

end 